\title{
Unveiling the photophysical properties of Boron- dipyrromethene dyes using a new accurate excited state coupled cluster method
}

\author{
Romain Berraud-Pache, Frank Neese, Giovanni Bistoni and Róbert Izsák* \\ Max-Planck-Institut für Kohlenforschung, Kaiser-Wilhelm Platz 1., 45470 Mülheim an der \\ Ruhr, Germany
}

\section{Computational details}

The convergence criteria are defined as follows:

- For the optimization, the tightopt threshold was selected corresponding to: Energy change $=1 \times 10^{-6}$ a.u., RMS gradient $=3 \times 10^{-5}$ a.u., MAX gradient $=1 \times 10^{-4}$ a.u., RMS step $=6 \times 10^{-4}$ a.u., MAX step $=1 \times 10^{-3}$ a.u.

- The GRID4 standard grid corresponds to a Lebedev angular grid with 302 points, a GaussChebyshev radial grid with a radial integration accuracy (IntAcc) of 4.67.

- The TightPNO threshold corresponds to the following cutoff values: $T_{\text {CutPairs }}=10^{-5}, T_{\text {CutDo }}=5 \mathrm{x}$ $10^{-3}, \mathrm{~T}_{\text {CutPNO }}=1.00 \times 10^{-7}, \mathrm{~T}_{\text {CutMKN }}=10^{-3}$ and the MP2 pair treatment is full iterative.

In ESD calculations, the 0-0 energy is printed at the end of the calculation. It is computed using the following formula:

$$
E^{0-0}=E^{A d i a}+\Delta E^{Z P E}
$$

with $E^{\text {Adia }}=E^{E S}\left(R^{E S}\right)-E^{G S}\left(R^{G S}\right)$, the difference of the electronic energies computed for the GS and $\mathrm{ES}$ at their geometries and $\Delta E^{Z P E}=E^{Z P E}\left(R^{E S}\right)-E^{Z P E}\left(R^{G S}\right)$, the difference of zero-point vibrational energy between the GS and ES. The computed 0-0 energies are very close to the energies of the most intense band of the absorption spectra. 
Table S1. Comparison of geometries obtained with various methods for a more flexible dye, Bo.21. The geometries were optimized using the def2-TZVP basis set. Vertical absorption energies were computed using DLPNO-STEOM-CCSD, the def2-TZVP basis set and TIGHTPNO. The DLPNO$\operatorname{CCSD}(\mathrm{T})$ calculations were carried out using the def2-TZVP basis set and TIGHTPNO. All values are relative to B3LYP results, the B3LYP vertical excitation energy being $16486 \mathrm{~cm}^{-1}$.

\begin{tabular}{|c|c|c|c|c|c|}
\hline BODIPY 21 & B3LYP & PBEO & M06-2X & $\omega B 97 X D$ & MP2 \\
\hline $\operatorname{RMSD}(\AA ̊)$ & 0 & 0.14 & 0.24 & 0.42 & 0.24 \\
\hline $\begin{array}{l}\text { Vertical excitation energies } \\
\qquad\left(\mathrm{cm}^{-1}\right)\end{array}$ & 0 & -1 & 44 & 537 & 103 \\
\hline DLPNO-CCSD(T) energy (kcal) & 0 & 1.385 & 5.678 & -0.583 & -1.949 \\
\hline
\end{tabular}

Table S2. Parameters describing the solvent in calculations with the CPCM implicit solvent model.

\begin{tabular}{ccc} 
Solvent & Dielectric constant $(\varepsilon)$ & Refractive Index \\
\hline Chloroform & 4.81 & 1.4458 \\
Cyclohexane & 2.02 & 1.4262 \\
\hline
\end{tabular}

Dichloromethane

8.93

1.4241

Ethanol

24.5

1.3614

Ethyl Acetate

6.02

1.3724

Methanol

32.7

1.3284

$\mathrm{N}-H e x a n e$

1.89

1.3758

Tetrahydrofuran

7.58

1.4072

Toluene 
Table S3. Experimental and computed excitation energies in $\mathrm{eV}$ for each BODIPY dye in the test set.

\begin{tabular}{|c|c|c|c|c|c|}
\hline $\begin{array}{l}\text { BODIPY } \\
\text { number }\end{array}$ & STEOM & $\begin{array}{l}\text { STEOM } \\
\text {-CPCM }\end{array}$ & $\begin{array}{c}\text { TDDFT-CPCM } \\
\text { B3LYP }\end{array}$ & $\begin{array}{l}\text { TDDFT } \\
\text { B3LYP }\end{array}$ & $\begin{array}{c}\text { Experiment } \\
\text { Solvent }\end{array}$ \\
\hline 1 & 3.183 & 3.134 & 3.287 & 3.364 & $\begin{array}{l}3.123^{1} \\
\mathrm{CH}_{2} \mathrm{Cl}_{2}\end{array}$ \\
\hline 2 & 2.900 & 2.845 & 3.105 & 3.123 & $\begin{array}{l}2.774^{1} \\
\mathrm{CH}_{2} \mathrm{Cl}_{2}\end{array}$ \\
\hline 3 & 2.886 & 2.913 & 3.191 & 3.056 & $\begin{array}{l}2.774^{1} \\
\mathrm{CH}_{2} \mathrm{Cl}_{2}\end{array}$ \\
\hline 4 & 2.166 & 2.19 & 2.455 & 2.43 & $\begin{array}{l}2.206^{2} \\
\mathrm{CH}_{2} \mathrm{Cl}_{2}\end{array}$ \\
\hline 5 & 2.263 & 2.331 & 2.431 & 2.854 & $\begin{array}{c}2.331^{3} \\
\text { THF }\end{array}$ \\
\hline 6 & 2.133 & 2.131 & 2.593 & 2.596 & $\begin{array}{l}2.175^{3} \\
\mathrm{CHCl}_{3}\end{array}$ \\
\hline 7 & 2.026 & 2.043 & 2.417 & 2.448 & $\begin{array}{l}2.050^{3} \\
\mathrm{CHCl}_{3}\end{array}$ \\
\hline 8 & 2.012 & 2.021 & 2.388 & 2.421 & $\begin{array}{l}2.046^{3} \\
\mathrm{CHCl}_{3}\end{array}$ \\
\hline 9 & 2.140 & 2.148 & 2.578 & 2.608 & $\begin{array}{l}2.172^{3} \\
\mathrm{CHCl}_{3}\end{array}$ \\
\hline 10 & 1.905 & 1.902 & 2.301 & 2.328 & $\begin{array}{l}2.029^{3} \\
\mathrm{CHCl}_{3}\end{array}$ \\
\hline 11 & 1.836 & 1.775 & 2.216 & 2.243 & $\begin{array}{l}1.922^{3} \\
\mathrm{CHCl}_{3}\end{array}$ \\
\hline 12 & 1.785 & 1.806 & 2.181 & 2.207 & $\begin{array}{l}1.917^{3} \\
\mathrm{CHCl}_{3}\end{array}$ \\
\hline 13 & 2.463 & 2.455 & 3.08 & 3.129 & $\begin{array}{c}2.460^{4} \\
\text { Cyclohexane }\end{array}$ \\
\hline 14 & 2.930 & 3.035 & 3.384 & 3.379 & $\begin{array}{c}3.054^{5} \\
\text { Ethyl Acetate }\end{array}$ \\
\hline 15 & 2.417 & 2.434 & 2.843 & 2.872 & $\begin{array}{c}2.357^{6} \\
\text { Cyclohexane }\end{array}$ \\
\hline 16 & 2.324 & 2.342 & 2.867 & 2.95 & $\begin{array}{l}2.515^{7-8} \\
\text { Methanol }\end{array}$ \\
\hline 17 & 2.305 & 2.307 & 2.878 & 2.883 & $\begin{array}{l}2.403^{9} \\
\mathrm{CH}_{2} \mathrm{Cl}_{2}\end{array}$ \\
\hline
\end{tabular}




\begin{tabular}{|c|c|c|c|c|c|}
\hline 18 & 2.113 & 2.184 & 2.509 & 2.535 & $\begin{array}{l}2.175^{9} \\
\mathrm{CH}_{2} \mathrm{Cl}_{2}\end{array}$ \\
\hline 19 & 2.353 & 2.382 & 2.868 & 2.892 & $\begin{array}{l}2.389^{10} \\
\mathrm{CH}_{2} \mathrm{Cl}_{2}\end{array}$ \\
\hline 20 & 2.204 & 2.192 & 2.61 & 2.641 & $\begin{array}{l}2.234^{11} \\
\text { Toluene }\end{array}$ \\
\hline 21 & 2.020 & 2.044 & 2.446 & 2.441 & $\begin{array}{c}2.199^{12} \\
\text { Methanol }\end{array}$ \\
\hline 22 & 1.848 & 1.851 & 2.219 & 2.24 & $\begin{array}{l}1.934^{13} \\
\mathrm{CH}_{2} \mathrm{Cl}_{2}\end{array}$ \\
\hline 23 & 2.326 & 2.341 & 2.704 & 2.748 & $\begin{array}{l}2.288^{14} \\
\mathrm{CH}_{2} \mathrm{Cl}_{2}\end{array}$ \\
\hline 24 & 2.03 & 2.018 & 2.36 & 2.387 & $\begin{array}{c}2.026^{14} \\
\mathrm{CH}_{2} \mathrm{Cl}_{2}\end{array}$ \\
\hline 25 & 2.133 & 2.137 & 2.673 & 2.683 & $\begin{array}{c}2.142^{15} \\
\mathrm{CHCl}_{3}\end{array}$ \\
\hline 26 & 3.125 & 3.223 & 3.315 & 3.306 & $\begin{array}{l}3.139^{16} \\
\mathrm{CH}_{2} \mathrm{Cl}_{2}\end{array}$ \\
\hline 27 & 2.816 & 2.874 & 3.047 & 3.031 & $\begin{array}{l}2.731^{16} \\
\mathrm{CH}_{2} \mathrm{Cl}_{2}\end{array}$ \\
\hline 28 & 2.894 & 2.918 & 2.741 & 3.143 & $\begin{array}{c}2.756^{17} \\
\text { N-hexane }\end{array}$ \\
\hline 29 & 1.834 & 1.824 & 2.234 & 2.247 & $\begin{array}{l}1.920^{11} \\
\text { Toluene }\end{array}$ \\
\hline 30 & 1.636 & 1.637 & 2.015 & 2.02 & $\begin{array}{l}1.744^{18} \\
\mathrm{CH}_{2} \mathrm{Cl}_{2}\end{array}$ \\
\hline 31 & 3.147 & 3.146 & 3.044 & 3.187 & $\begin{array}{c}3.077^{19} \\
\mathrm{CH}_{2} \mathrm{Cl}_{2}\end{array}$ \\
\hline 32 & 2.851 & 2.755 & 2.107 & 2.789 & $\begin{array}{c}2.661^{19} \\
\mathrm{CH}_{2} \mathrm{Cl}_{2}\end{array}$ \\
\hline 33 & 2.866 & 2.864 & 2.784 & 2.873 & $\begin{array}{l}2.904^{19} \\
\mathrm{CH}_{2} \mathrm{Cl}_{2}\end{array}$ \\
\hline 34 & 2.703 & 2.656 & 2.269 & 2.719 & $\begin{array}{l}2.616^{19} \\
\mathrm{CH}_{2} \mathrm{Cl}_{2}\end{array}$ \\
\hline 35 & 1.602 & 1.625 & 1.866 & 1.866 & $\begin{array}{c}1.636^{20} \\
\mathrm{CHCl}_{3}\end{array}$ \\
\hline 36 & 1.711 & 1.741 & 2.136 & 2.137 & $\begin{array}{l}1.792^{20} \\
\mathrm{CHCl}_{3}\end{array}$ \\
\hline
\end{tabular}




\begin{tabular}{|c|c|c|c|c|c|}
\hline 37 & 2.387 & 2.333 & 2.795 & 2.857 & $\begin{array}{l}2.362^{21} \\
\text { Toluene }\end{array}$ \\
\hline 38 & 2.292 & 2.322 & 2.626 & 2.67 & $\begin{array}{c}2.344^{22} \\
\text { Cyclohexane }\end{array}$ \\
\hline 39 & 2.002 & 1.984 & 2.491 & 2.525 & $\begin{array}{c}2.088^{22} \\
\text { Cyclohexane }\end{array}$ \\
\hline 40 & 2.024 & 2.363 & 2.605 & 2.665 & $\begin{array}{l}2.242^{23} \\
\mathrm{CH}_{2} \mathrm{Cl}_{2}\end{array}$ \\
\hline 41 & 1.916 & 1.902 & 2.171 & 2.268 & $\begin{array}{l}1.953^{23} \\
\mathrm{CH}_{2} \mathrm{Cl}_{2}\end{array}$ \\
\hline 42 & 3.86 & 3.912 & 3.788 & 3.85 & $\begin{array}{l}3.713^{7} \\
\mathrm{CH}_{2} \mathrm{Cl}_{2}\end{array}$ \\
\hline 43 & 3.582 & 3.7 & 3.521 & 3.434 & $\begin{array}{l}3.493^{7} \\
\text { Ethanol }\end{array}$ \\
\hline 44 & 2.297 & 2.341 & 2.316 & 2.574 & $\begin{array}{l}2.385^{24} \\
\text { Toluene }\end{array}$ \\
\hline 45 & 2.215 & 2.228 & 2.559 & 2.6 & $\begin{array}{l}2.288^{24} \\
\text { Toluene }\end{array}$ \\
\hline 46 & 2.075 & 2.07 & 2.4 & 2.42 & $\begin{array}{l}2.036^{25} \\
\mathrm{CH}_{2} \mathrm{Cl}_{2}\end{array}$ \\
\hline 47 & 1.756 & 1.803 & 1.986 & 2.011 & $\begin{array}{l}1.824^{25} \\
\mathrm{CH}_{2} \mathrm{Cl}_{2}\end{array}$ \\
\hline
\end{tabular}

Table S4. Parameters used in the ESD calculation for the Bo.5 dye. The same parameters are used for all the three algorithms studied (VG, AHAS and AH).

\begin{tabular}{|lc|}
\hline Requested calculation: & absorption \\
Coordinate system: & Deloc. Internal (BAKER) \\
Lineshape function: & Gaussian \\
Homogeneous linewidht is: & $50.00 \mathrm{~cm}-1$ \\
Inhomogeneous linewidht is: & $250.00 \mathrm{~cm}-1$ \\
Number of points: & 131072 \\
Maximum time: & $1592.65 \mathrm{fs}$ \\
Spectral resolution: & $3.33 \mathrm{~cm}-1$ \\
Temperature used: & $298.15 \mathrm{~K}$ \\
Z value: & $1.014323 \mathrm{e}-96$ \\
Cutoff for the correlation function: $\quad 1.00 \mathrm{e}-06$ \\
Last element of the correlation function: $0.000000,0.000000$ \\
\hline
\end{tabular}

The displacement vector can be defined as $K=L_{x}^{T}\left(\bar{q}_{0}-q_{0}\right)$ and the Duchinsky rotation matrix as $J=L_{x}^{T} \bar{L}_{x}$ with $L_{x}^{T}$ being the eigenvectors of the mass-weighted Hessian, $\bar{L}_{x}$ the eigenvectors of the mass-weighted Hessian of the final geometry and $\bar{q}_{0}\left(q_{0}\right)$ the mass-weighted Cartesian nuclear 
coordinates of the final structure (initial structure). The displacement vectors are defined in mass weighted atomic units, $\boldsymbol{a}_{\mathbf{0}} \sqrt{\boldsymbol{m}_{\boldsymbol{e}}}$ with $\boldsymbol{a}_{\mathbf{0}}$ the bohr radius and $\boldsymbol{m}_{\boldsymbol{e}}$ the mass of the electron.

It is sometimes also convenient to use dimensionless coordinates defined as $Q_{\alpha}=\sqrt{\frac{\omega_{\alpha}}{\hbar}} q_{\alpha}$ with $\omega_{\alpha}$ being the frequency of the mode $\alpha$. We will use dimensionless $\mathrm{K}$ vectors and $\mathrm{K}{ }^{*} \mathrm{~K}$ (norm square of the displacement vector) values in the tables below.

Table S5. ESD parameters for Bo.5. $\mathrm{K} * \mathrm{~K}$ (dimensionless)corresponds to the norm square of the displacement vector, the RMSD computed between the GS and $1^{\text {st }} \mathrm{ES}$ structures is also provided. The diagonality index (DI) is computed for the Duchinsky J matrix J as: DI $=\sqrt{\sum_{i} J_{i i}^{2} / \sum_{i j} J_{i j}^{2}}$.

$\begin{array}{cccc}\text { BODIPY 5 } & \text { AH } & \text { AHAS } & \text { VG } \\ \text { K*K } & 2.35 & 2.17 & 2.1 \\ \text { RMSD }(\AA) & 0.2350 & 0.0516 & 0.0711 \\ \text { Diagonality index } & 0.620983 & 0.643773 & 0.999423 \\ \text { Maximum absorption }\left(\mathrm{cm}^{-1}\right) & 19599 & 18470 & 18636 \\ \text { Maximum fluorescence }\left(\mathrm{cm}^{-1}\right) & 18300 & 17695 & 17534\end{array}$

Table S6. Displacement vector K (dimensionless) for Bo.5 with the VG, AHAS or AH algorithms.

\begin{tabular}{|c|c|c|c|c|c|}
\hline \multicolumn{3}{|c|}{ The $\mathrm{K}$ vector for VG (dimensionless) is: } & \multicolumn{3}{|c|}{ The $\mathrm{K}$ vector for AHAS(dimensionless) is: } \\
\hline mode & e displace & nt Qf corresp. mode & $\bmod$ & e displacer & nt Qf corresp. mode \\
\hline 1 & -0.007402 & 1: 1.00 & 1 & -0.008768 & 1: 0.94; \\
\hline 2 & 0.002131 & $2: 1.00$ & 2 & -0.001964 & 2: 0.62 \\
\hline 3 & 0.012622 & 3: 0.99 & 3 & 0.007836 & 3: 0.62 \\
\hline 4 & 0.014483 & 4: 0.99 & 4 & 0.014846 & 4: 0.66; \\
\hline 5 & -0.000938 & $5: 1.00$ & 5 & 0.000108 & 7: 0.70; \\
\hline 6 & -0.128950 & $6: 1.00$ & 6 & -0.051723 & 6: 0.85 \\
\hline 7 & -0.037671 & $7: 1.01$ & 7 & -0.033395 & 5: 0.53 \\
\hline 8 & -0.242364 & $8: 1.01$ & 8 & -0.267629 & 9: 0.71; \\
\hline 9 & -0.043366 & $9: 1.00$ & 9 & -0.046932 & \\
\hline 10 & 0.005992 & $10: 1.02$ & 10 & 0.004333 & 10: 0.41 \\
\hline 11 & 0.013429 & 11: 0.99; & 11 & 0.023169 & 11: 0.62; \\
\hline 12 & 0.030195 & 12: 1.00 & 12 & 0.036777 & 12: 0.65 \\
\hline 13 & 0.184157 & 13: 0.99 & 13 & 0.178246 & 13: $0.36 ; 14: 0.58$ \\
\hline 14 & -0.022158 & 14: 1.01 & 14 & -0.042283 & 13: 0.44 \\
\hline 15 & -0.042795 & $15: 1.02$ & 15 & 0.007171 & 15: 0.76 \\
\hline 16 & 0.187405 & 16: 0.98 & 16 & 0.183804 & 16: 0.79; \\
\hline 17 & 0.246607 & 17: 1.00; & 17 & 0.249953 & 17: 0.77; \\
\hline 18 & 0.011904 & 18: 1.00; & 18 & 0.020054 & 18: 0.64; \\
\hline 19 & 0.230567 & 19: 1.00; & 19 & 0.217860 & 20: 0.70; \\
\hline 20 & -0.038128 & $20: 1.02$ & 20 & -0.025353 & 19: 0.58 \\
\hline 21 & 0.092878 & $21: 1.01$ & 21 & 0.113338 & 21: 0.50 \\
\hline 22 & -0.281111 & $22: 1.01$ & 22 & -0.294140 & 22: 0.81 \\
\hline 23 & 0.415809 & 23: 0.98; & 23 & 0.427007 & 23: 0.64; \\
\hline
\end{tabular}




\begin{tabular}{|c|c|c|c|c|c|}
\hline 24 & -0.182513 & 24: 1.00; & 24 & -0.189332 & 24: 0.73 \\
\hline 25 & 0.310388 & 25: 1.01 & 25 & 0.345938 & $25: 0.51 ; 26: 0.39$ \\
\hline 26 & -0.056953 & 26: 1.01; & 26 & -0.026341 & $28: 0.70$ \\
\hline 27 & 0.008612 & 27: 1.00; & 27 & 0.008040 & 27: 0.49; \\
\hline 28 & 0.008963 & 28: 1.00 & 28 & 0.009051 & 29: 0.44; \\
\hline 29 & 0.019842 & 29: 1.00; & 29 & 0.021116 & 31: 0.63 \\
\hline 30 & 0.020916 & 30: 0.99; & 30 & 0.017941 & 33: 0.31; \\
\hline 31 & 0.077178 & $31: 1.00$ & 31 & 0.068070 & \\
\hline 32 & -0.355837 & 32: 1.00 & 32 & -0.371104 & 32: 0.70 \\
\hline 33 & -0.018419 & 33: 0.99; & 33 & -0.019088 & 34: 0.43 \\
\hline 34 & -0.008749 & 34: 1.00; & 34 & -0.006299 & 37: 0.86; \\
\hline 35 & 0.011537 & $35: 1.00$ & 35 & 0.012865 & 35: 0.77; \\
\hline 36 & 0.000591 & 36: 1.01; & 36 & 0.001779 & 36: 0.99; \\
\hline 37 & -0.002686 & 37: 1.00; & 37 & -0.008605 & 38: 0.89 \\
\hline 38 & -0.000598 & 38: 0.98 & 38 & 0.000319 & 39: 0.87; \\
\hline 39 & 0.164413 & 39: 0.99; & 39 & 0.153356 & 41: 0.80 \\
\hline 40 & 0.067256 & 40: 0.99; & 40 & 0.063593 & 30: 0.31 \\
\hline 41 & 0.322075 & 41: 1.01; & 41 & 0.354740 & 40: 0.81; \\
\hline 42 & -0.139372 & 42: 1.00; & 42 & -0.132171 & 42: $0.56 ; 43: 0.37$ \\
\hline 43 & 0.006325 & 43: 1.00; & 43 & -0.008076 & 42: $0.34 ; 43: 0.60$ \\
\hline 44 & -0.221323 & 44: 0.99; & 44 & -0.223899 & $44: 0.88$ \\
\hline 45 & 0.004366 & 45: 1.00; & 45 & 0.002404 & 45: 0.78; \\
\hline 46 & 0.001484 & 46: 1.00; & 46 & 0.003482 & 46: 0.79; \\
\hline 47 & 0.050550 & 47: 1.00; & 47 & 0.052644 & 47: 0.61; \\
\hline 48 & 0.120012 & 48: 1.00; & 48 & 0.125854 & 48: 0.57; \\
\hline 49 & 0.190071 & 49: 1.00; & 49 & 0.190581 & 49: 0.83; \\
\hline 50 & -0.069815 & 50: 1.00; & 50 & -0.077347 & 50: 0.98; \\
\hline 51 & -0.098938 & 51: 1.00; & 51 & -0.112042 & 51: 0.65 \\
\hline 52 & -0.377937 & 52: 1.00; & 52 & -0.396040 & 52: 0.59; \\
\hline 53 & 0.201590 & 53: 1.00 & 53 & 0.209739 & 54: 0.76; \\
\hline 54 & -0.265746 & 54: 1.00; & 54 & -0.272872 & 53: 0.84 \\
\hline 55 & 0.024409 & 55: 1.00; & 55 & 0.027844 & 55: 0.34; 56: 0.50; \\
\hline 56 & -0.088996 & 56: 1.00; & 56 & -0.094975 & 55: 0.48 \\
\hline 57 & -0.144314 & 57: 1.01; & 57 & -0.132794 & \\
\hline 58 & 0.089495 & 58: 1.00; & 58 & 0.095858 & 58: 0.54; \\
\hline 59 & 0.231439 & 59: 1.00; & 59 & 0.232591 & 60: 0.43; \\
\hline 60 & 0.108122 & 60: 1.00; & 60 & 0.104639 & 61: 0.86; \\
\hline 61 & 0.008322 & 61: 1.00; & 61 & 0.005259 & 62: 0.49; \\
\hline 62 & -0.046921 & 62: 1.00; & 62 & -0.046822 & 62: 0.45 \\
\hline 63 & 0.386523 & 63: 1.00; & 63 & 0.388383 & 65: 0.53; \\
\hline 64 & 0.506479 & 64: 1.00 & 64 & 0.500816 & 65: 0.39; \\
\hline 65 & -0.150978 & 65: 1.00; & 65 & -0.149777 & 66: 0.97; \\
\hline 66 & -0.023446 & 66: 1.00; & 66 & -0.022977 & 67: 1.00; \\
\hline 67 & -0.213290 & 67: 1.00; & 67 & -0.210093 & 68: 0.81 \\
\hline 68 & -0.025893 & 68: 1.01; & 68 & -0.022809 & 64: 0.62 \\
\hline 69 & -0.060342 & 69: 1.00; & 69 & -0.063964 & 69: 0.57; 70: 0.37; \\
\hline 70 & 0.101960 & 70: 1.00; & 70 & 0.103127 & 72: 0.47; \\
\hline 71 & 0.265707 & 71: 1.00 & 71 & 0.256259 & 71: 0.39; \\
\hline 72 & -0.250268 & 72: 0.99; & 72 & -0.240033 & 71: 0.34; 72: 0.34; \\
\hline 73 & 0.217717 & 73: 1.00; & 73 & 0.218109 & 73: 0.75; \\
\hline 74 & -0.019916 & 74: 1.00; & 74 & -0.020338 & 74: 0.99; \\
\hline 75 & 0.015711 & 75: 1.00; & 75 & 0.015312 & 75: 0.99; \\
\hline 76 & 0.001566 & 76: 1.00; & 76 & 0.000998 & 76: 0.99; \\
\hline 77 & 0.002457 & 77: 1.00; & 77 & 0.004310 & 77: 1.00; \\
\hline 78 & -0.001091 & 78: 1.00; & 78 & -0.002454 & 78: 1.00; \\
\hline 79 & 0.005819 & 79: 1.00; & 79 & 0.006549 & 79: 0.99; \\
\hline
\end{tabular}




\begin{tabular}{|c|c|c|c|c|c|}
\hline 80 & 0.045873 & 80: 1.00 & 80 & 0.043958 & 81: $0.31 ; 82: 0.69$ \\
\hline 81 & -0.035627 & 81: 1.00 & 81 & -0.033838 & 81: $0.46 ; 84: 0.33$ \\
\hline 82 & 0.001653 & 82: 1.00; & 82 & 0.001328 & 80: 1.00 \\
\hline 83 & 0.041720 & 83: 1.00 & 83 & 0.036643 & 84: 0.65 \\
\hline 84 & 0.023141 & 84: 1.00 & 84 & 0.019032 & 83: 0.96; \\
\hline \multicolumn{6}{|c|}{ The K vector for $\mathrm{AH}$ (dimensionless) is: } \\
\hline \multicolumn{6}{|c|}{ mode displacement Qf corresp. mode } \\
\hline 1 & -0.009477 & 1: 0.77 & & & \\
\hline 2 & 0.007602 & 2: 0.38 & & & \\
\hline 3 & 0.032821 & $6: 0.55$ & & & \\
\hline 4 & 0.024107 & 2: 0.54 & & & \\
\hline 5 & -0.000594 & 7: 0.82; & & & \\
\hline 6 & -0.603819 & 4: $0.52 ; 5: 0.41$ & & & \\
\hline 7 & -0.110922 & & & & \\
\hline 8 & -0.394857 & 9: 0.77 & & & \\
\hline 9 & -0.085479 & 8: 0.30; 10: 0.43; & & & \\
\hline 10 & 0.058111 & 10: 0.38 & & & \\
\hline 11 & -0.043724 & 11: 0.57 & & & \\
\hline 12 & -0.012015 & 12: 0.76 & & & \\
\hline 13 & 0.374264 & 13: $0.39 ; 14: 0.51$ & & & \\
\hline 14 & -0.012320 & 13: 0.43 & & & \\
\hline 15 & -0.093012 & 15: 0.74 & & & \\
\hline 16 & 0.137606 & 16: $0.50 ; 17: 0.34$ & & & \\
\hline 17 & 0.193850 & 17: 0.62 & & & \\
\hline 18 & -0.061480 & 18: 0.70 & & & \\
\hline 19 & 0.338335 & 20: 0.80 & & & \\
\hline 20 & -0.108511 & 19: 0.61; & & & \\
\hline 21 & 0.016517 & 21: 0.57 & & & \\
\hline 22 & -0.350595 & 22: 0.73 & & & \\
\hline 23 & 0.422844 & 23: 0.84 & & & \\
\hline 24 & -0.222624 & 24: 0.90; & & & \\
\hline 25 & 0.238171 & $25: 0.86$ & & & \\
\hline 26 & -0.184791 & 27: 0.71; & & & \\
\hline 27 & 0.003315 & 28: 0.41 & & & \\
\hline 28 & -0.007485 & & & & \\
\hline 29 & -0.034592 & 31: 0.79; & & & \\
\hline 30 & 0.032240 & & & & \\
\hline 31 & 0.126982 & 33: 0.35 & & & \\
\hline 32 & -0.330092 & 32: 0.79 & & & \\
\hline 33 & -0.020840 & 34: 0.50 & & & \\
\hline 34 & 0.004760 & $37: 0.85$ & & & \\
\hline 35 & -0.008665 & 35: 0.80 & & & \\
\hline 36 & -0.003023 & 36: 1.00; & & & \\
\hline 37 & 0.015515 & 38: 0.91 & & & \\
\hline 38 & -0.003628 & 39: 0.89 & & & \\
\hline 39 & 0.174225 & 40: 0.38; 41: 0.47; & & & \\
\hline 40 & 0.023236 & 30: 0.31 & & & \\
\hline 41 & 0.309268 & 40: 0.43; 41: 0.40; & & & \\
\hline 42 & -0.143288 & 42: 0.57; 43: 0.32; & & & \\
\hline 43 & -0.023673 & 42: 0.30; 43: 0.62; & & & \\
\hline 44 & -0.238568 & 44: 0.82 & & & \\
\hline 45 & 0.046797 & 45: 0.87 & & & \\
\hline 46 & 0.050681 & 46: 0.85 & & & \\
\hline 47 & 0.002827 & 47: 0.45 & & & \\
\hline
\end{tabular}




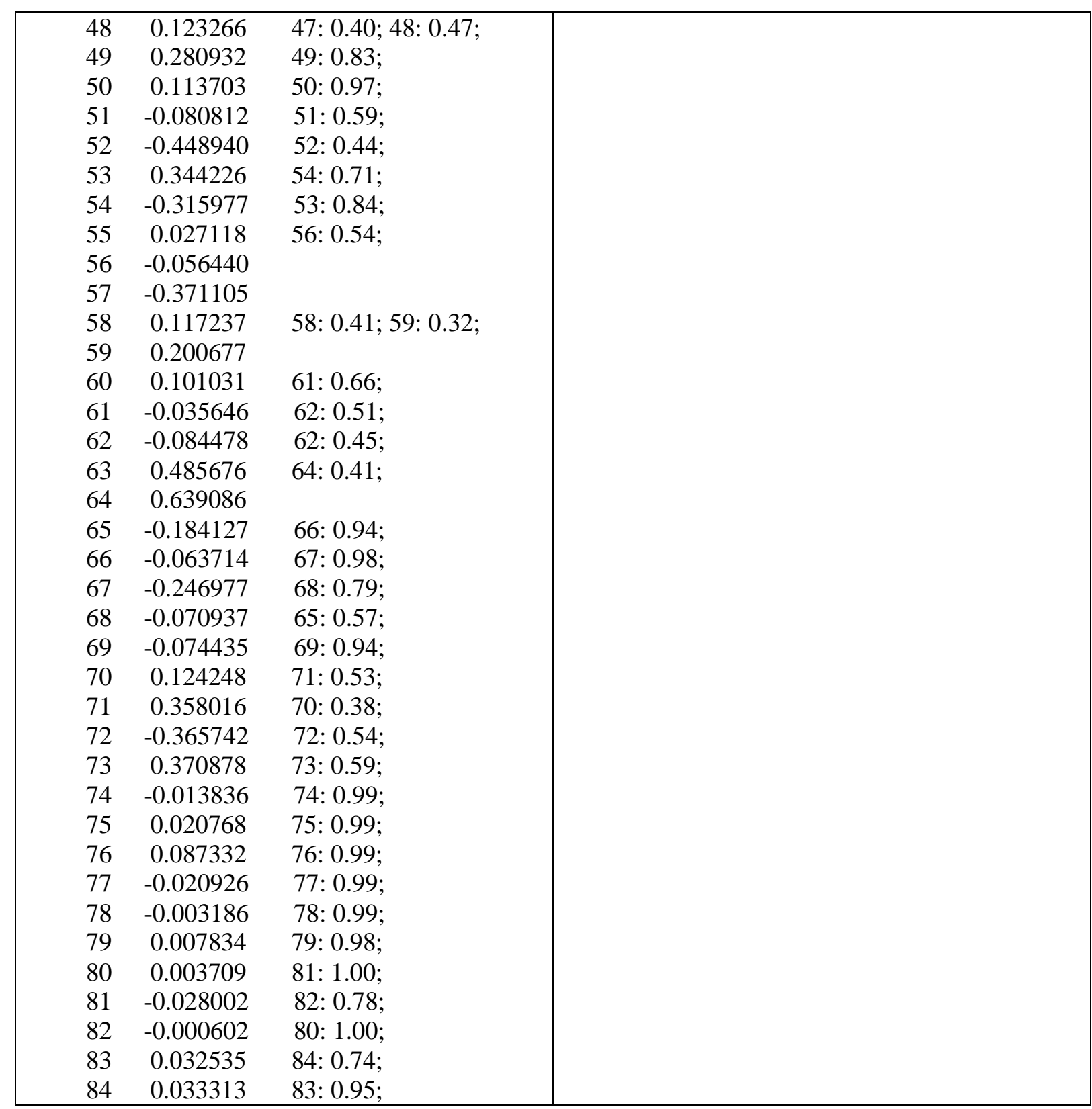




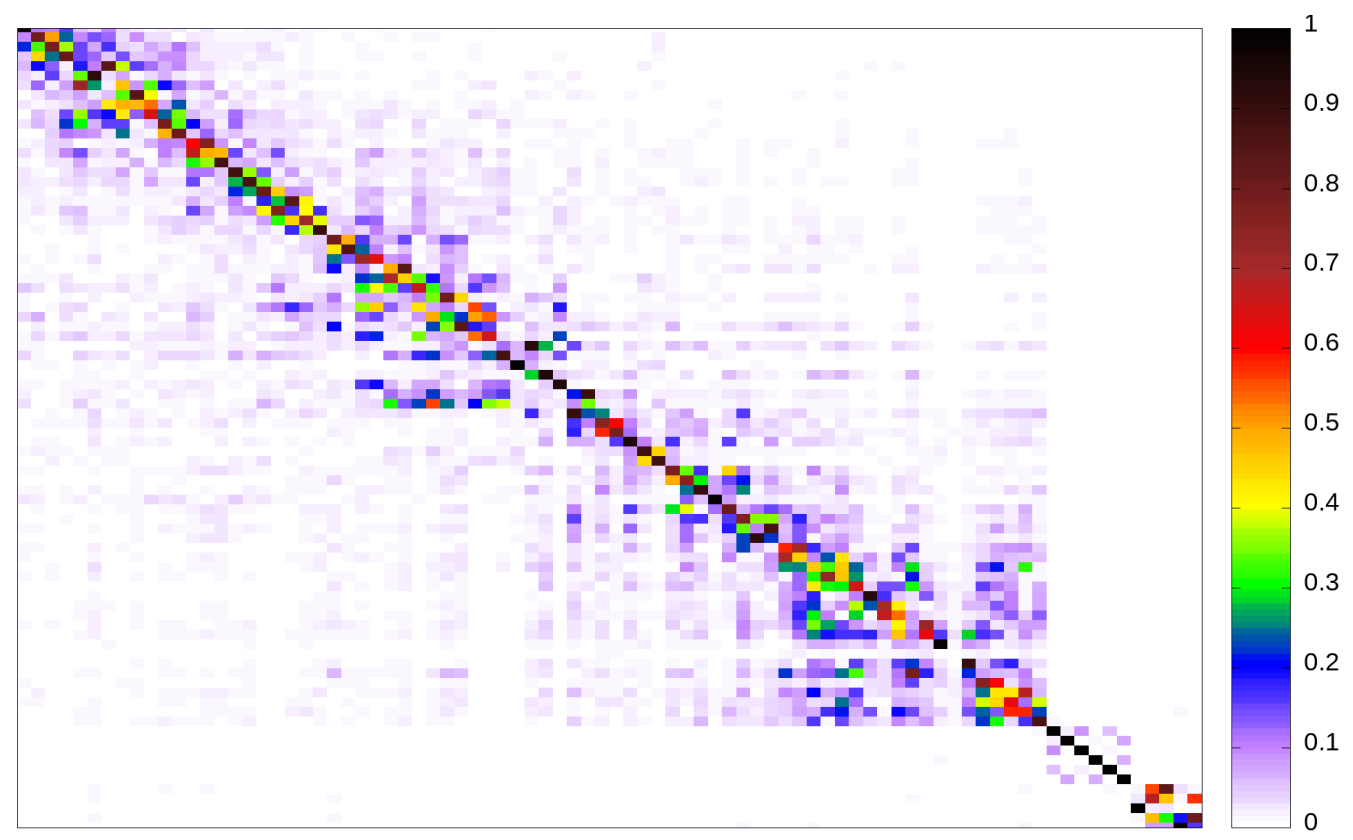

Figure S1. Duschinsky matrix for the Bo.5 dye using the AHAS algorithm.

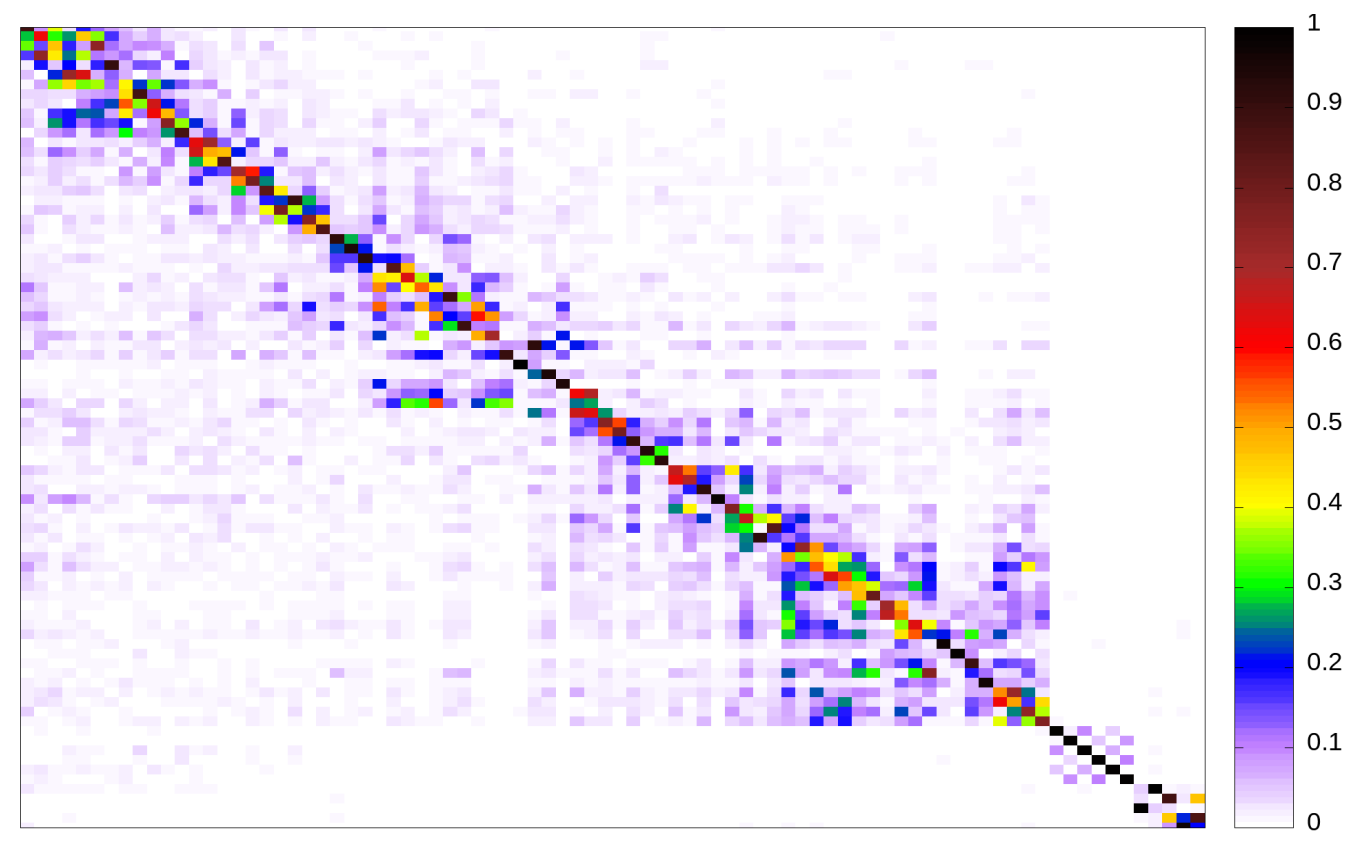

Figure S2. Duschinsky matrix for the Bo.5 dye using the AH algorithm. 


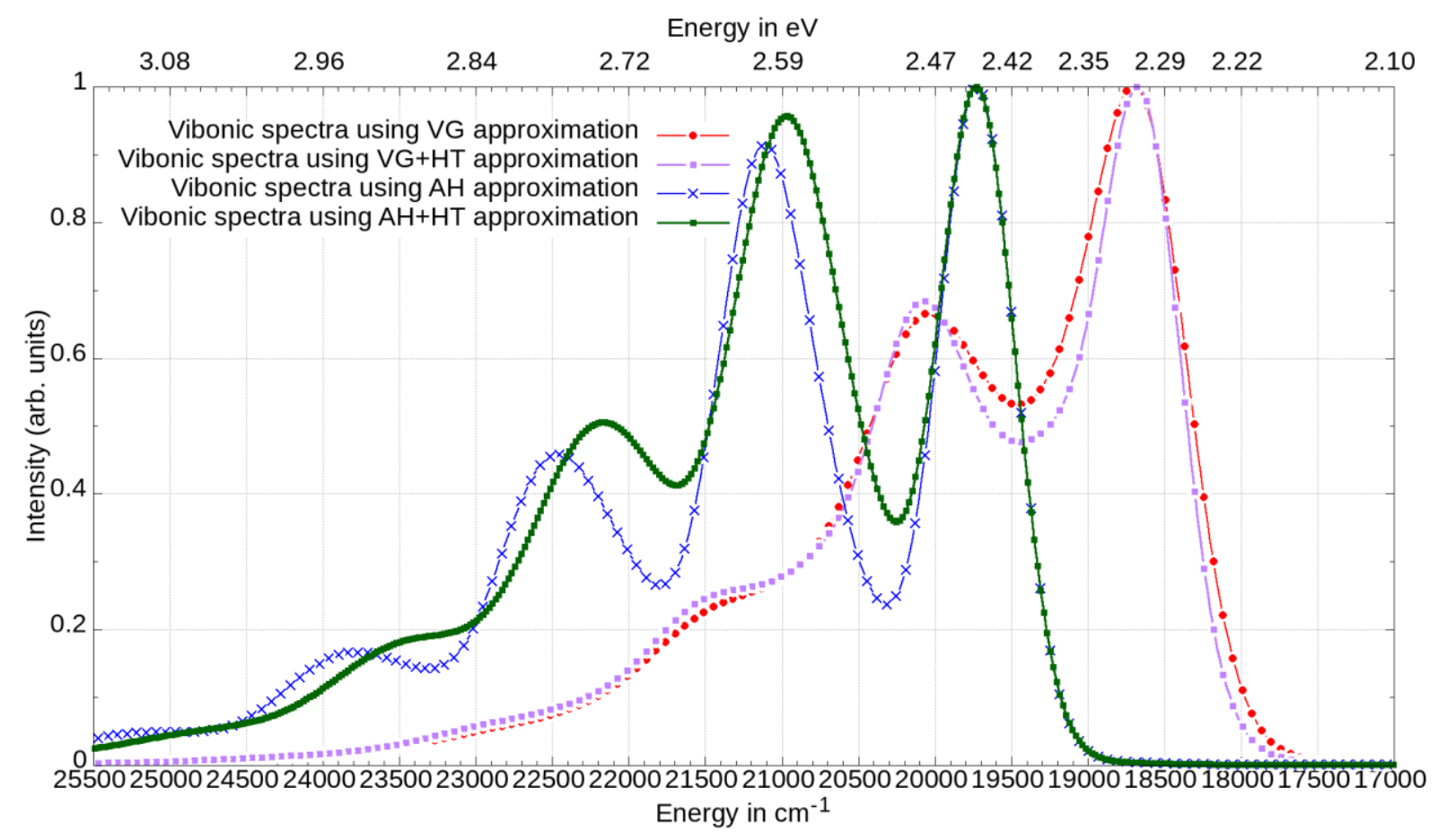

Figure S3. Comparison between the computed vibronic spectra with and without the inclusion of Herzberg-Teller effects (HT) for the VG and AH algorithms. The Gaussian lineshape was used for the computed spectra with the default linewidth parameter of $250 \mathrm{~cm}^{-1}$. 
Table S7. The square norm of the displacement vector $\mathrm{K}^{*} \mathrm{~K}$ computed using the VG algorithm for all dyes in the test set. The value of $\mathrm{K}^{*} \mathrm{~K}$ is considered too large if it is above 8 , in which case the calculation is aborted. The difference of vertical excitation energies $\left(\lambda_{\text {vert }}\right)$ and band maxima $\left(\lambda_{\max }\right)$ are also provided in $\mathrm{eV}$ units. The MAE for the whole benchmark set is $0.00840 \mathrm{eV}$.

\begin{tabular}{|c|c|c|c|c|c|c|c|c|c|}
\hline BODIPY & 1 & 2 & 3 & 4 & 5 & 6 & 7 & 8 & 9 \\
\hline $\mathrm{K}^{*} \mathrm{~K}$ & 3.220 & 1.490 & 1.651 & 1.152 & 2.100 & 0.932 & 0.783 & 0.772 & 0.824 \\
\hline $\begin{array}{c}\lambda \max -\lambda \text { vert } \\
(\mathrm{eV})\end{array}$ & 0.00045 & 0.00120 & 0.00331 & 0.00438 & -0.03794 & 0.00365 & 0.00335 & 0.00198 & 0.00483 \\
\hline BODIPY & 10 & 11 & 12 & 13 & 14 & 15 & 16 & 17 & 18 \\
\hline $\mathrm{K} * \mathrm{~K}$ & 1.068 & 0.735 & 0.698 & 0.835 & 0.915 & 1.013 & 0.769 & 0.634 & 1.131 \\
\hline $\begin{array}{c}\lambda \max -\lambda \text { vert } \\
(\mathrm{eV})\end{array}$ & 0.00672 & 0.06100 & 0.00404 & -0.00353 & 0.00337 & 0.00968 & 0.00559 & 0.00424 & 0.00258 \\
\hline BODIPY & 19 & 20 & 21 & 22 & 23 & 24 & 25 & 26 & 27 \\
\hline $\mathrm{K}^{*} \mathrm{~K}$ & 2.878 & 2.468 & 1.837 & 0.970 & 0.900 & 0.787 & 0.544 & 1.765 & 1.391 \\
\hline $\begin{array}{c}\lambda \max -\lambda \text { vert } \\
(\mathrm{eV})\end{array}$ & 0.00714 & 0.01276 & 0.00755 & 0.00318 & 0.00659 & 0.00238 & 0.00284 & 0.00494 & 0.00412 \\
\hline BODIPY & 28 & 29 & 30 & 31 & 32 & 33 & 34 & 35 & 36 \\
\hline $\mathrm{K}^{*} \mathrm{~K}$ & 1.245 & 0.954 & 0.746 & 2.589 & 3.155 & 3.666 & 2.437 & 1.380 & 0.759 \\
\hline $\begin{array}{c}\lambda \max -\lambda \text { vert } \\
(\mathrm{eV})\end{array}$ & -0.03988 & 0.00421 & 0.00424 & 0.00923 & 0.01589 & 0.01855 & 0.00841 & 0.00066 & 0.00411 \\
\hline BODIPY & 37 & 38 & 39 & 40 & 41 & 42 & 43 & 44 & 45 \\
\hline $\mathrm{K}^{*} \mathrm{~K}$ & 0.804 & 1.939 & 0.740 & 2.559 & 0.809 & 2.974 & 3.646 & 2.445 & 0.808 \\
\hline $\begin{array}{c}\lambda \max -\lambda \text { vert } \\
(\mathrm{eV})\end{array}$ & 0.00161 & 0.00741 & 0.00262 & 0.02355 & 0.00152 & 0.00931 & 0.00317 & 0.00659 & 0.00273 \\
\hline BODIPY & 46 & 47 & SMcy7 & PDT-1 & BD-1 & $\mathrm{BOP}$ & $Y-1$ & & \\
\hline $\mathrm{K}^{*} \mathrm{~K}$ & 1.123 & 3.195 & 1.63 & 0.83 & 0.75 & 1. & & & \\
\hline $\begin{array}{c}\lambda \max -\lambda \text { vert } \\
(\mathrm{eV})\end{array}$ & 0.00601 & -0.01634 & 0.00511 & .01423 & 00190 & 0.00 & & & \\
\hline
\end{tabular}

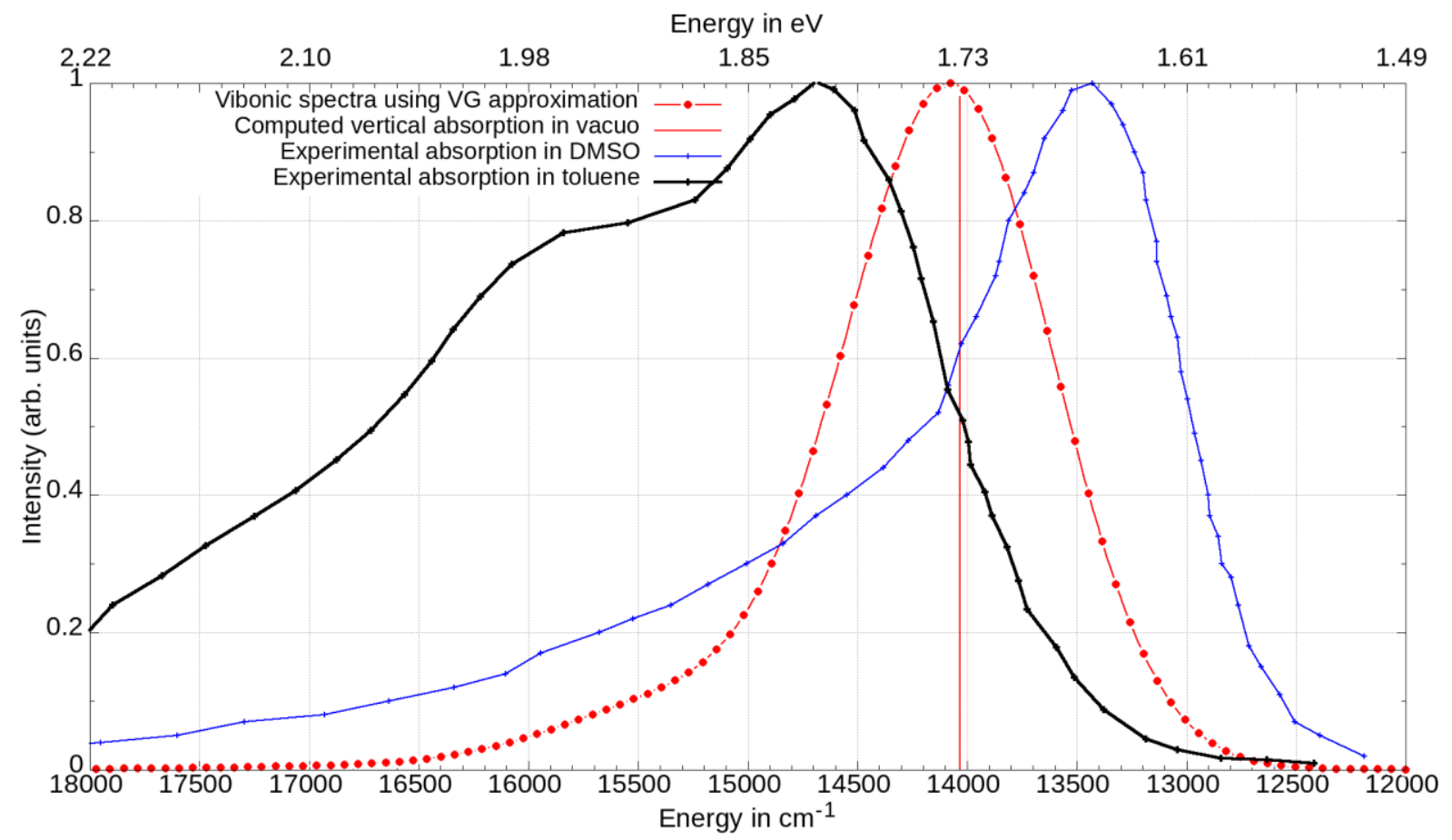

Figure S4. Comparison between the computed vertical excitation energy, vibronic spectra and experimental absorption spectra of SMCy7. The vibronic spectrum has been computed using in vacuo structures and vertical energies while experimental data are measured in toluene and DMSO. The 
shoulder observed in the experimental spectrum in toluene is much less prominent in the VG calculation. This might be due to neglecting mode mixing or solvent effects.

Table S8. Computed Mean Absolute Error in eV for the BODIPY dyes studied sorted according to the solvent model used. Calculations were carried out at the STEOM and STEOM-CPCM levels with the def2-TZVP basis set.

\begin{tabular}{|c|c|c|c|}
\hline MAE & STEOM & $\begin{array}{l}\text { STEOM- } \\
\text { CPCM }\end{array}$ & $\begin{array}{l}\text { Number } \\
\text { of } \\
\text { BODIPY }\end{array}$ \\
\hline $\mathrm{CH}_{2} \mathrm{Cl}_{2}$ & 0.079 & 0.068 & 22 \\
\hline $\mathrm{CHCl}_{3}$ & 0.060 & 0.055 & 10 \\
\hline THF & 0.068 & 0.000 & 1 \\
\hline Cyclohexane & 0.050 & 0.052 & 4 \\
\hline Ethyl Acetate & 0.124 & 0.019 & 1 \\
\hline Methanol & 0.185 & 0.164 & 2 \\
\hline n-Hexane & 0.138 & 0.162 & 1 \\
\hline Toluene & 0.050 & 0.054 & 5 \\
\hline Ethanol & 0.089 & 0.207 & 1 \\
\hline $\begin{array}{l}\text { Apolar } \\
\text { solvents }\end{array}$ & 0.072 & 0.062 & 44 \\
\hline $\begin{array}{l}\text { Polar } \\
\text { solvents }\end{array}$ & 0.153 & 0.178 & 3 \\
\hline
\end{tabular}


Table S9. Computed vertical absorption energies in eV of 4 large BODIPY dyes obtained with DLPNO-STEOM-CCSD in gas phase and CPCM with the def2-SVP, def2-TZVP(-f) and def2-TZVP basis sets. The calculations times correspond to a parallel run on 8 cores of an Intel(R) Xeon(R) CPU E5-2640v3@ 2.60GHz) processor.

\begin{tabular}{lllll}
\multicolumn{1}{c}{ BODIPY } & \multicolumn{5}{c}{ DLPNO-STEOM-CCSD } \\
& def2-SVP & def2-TZVP(-f) & def2-TZVP & Experiment \\
& 1.74 & 1.71 & 1.74 & \\
SMcy7 gas phase & $(1 \mathrm{~d} 17 \mathrm{~h})$ & $(8 \mathrm{~d} 1 \mathrm{~h})$ & $(15 \mathrm{~d} 7 \mathrm{~h})$ & 1.82 \\
SMcy7 CPCM & 1.44 & 1.45 & 1.48 & $($ Toluene $)$ \\
& 1.80 & 1.70 & 1.71 & \\
PDT-1 gas phase & $(14 \mathrm{~h})$ & $(2 \mathrm{~d} 15 \mathrm{~h})$ & $(4 \mathrm{~d} 4 \mathrm{~h})$ & 1.78 \\
& 1.78 & 1.69 & 1.70 & $\left(\mathrm{CH}_{2} \mathrm{Cl}_{2}\right)$ \\
PDT-1 CPCM & 1.14 & 1.07 & 1.09 & \\
BD-1 gas phase & $(2 \mathrm{~d} 4 \mathrm{~h})$ & $(6 \mathrm{~d} 7 \mathrm{~h})$ & $(16 \mathrm{~d} 8 \mathrm{~h})$ & 1.14 \\
& 1.14 & 1.06 & 1.06 & $\left(\mathrm{CH}_{2} \mathrm{Cl}_{2}\right)$ \\
BD-1 CPCM & 2.32 & 2.28 & 2.28 & \\
BOPHY-1 gas phase & $(3 \mathrm{~d} 6 \mathrm{~h})$ & $(8 \mathrm{~d} 9 \mathrm{~h})$ & $(26 \mathrm{~d} 15 \mathrm{~h})$ & 2.15 \\
& & $\left(\mathrm{CH}_{2} \mathrm{Cl}_{2}\right)$ \\
BOPHY-1 CPCM & 2.33 & 2.27 & 2.27 &
\end{tabular}


Table S10. Cartesian coordinates of the BODIPY dyes studied. Geometries were optimized at the DFT/D3-B3LYP level of theory with the def2-TZVP basis set.

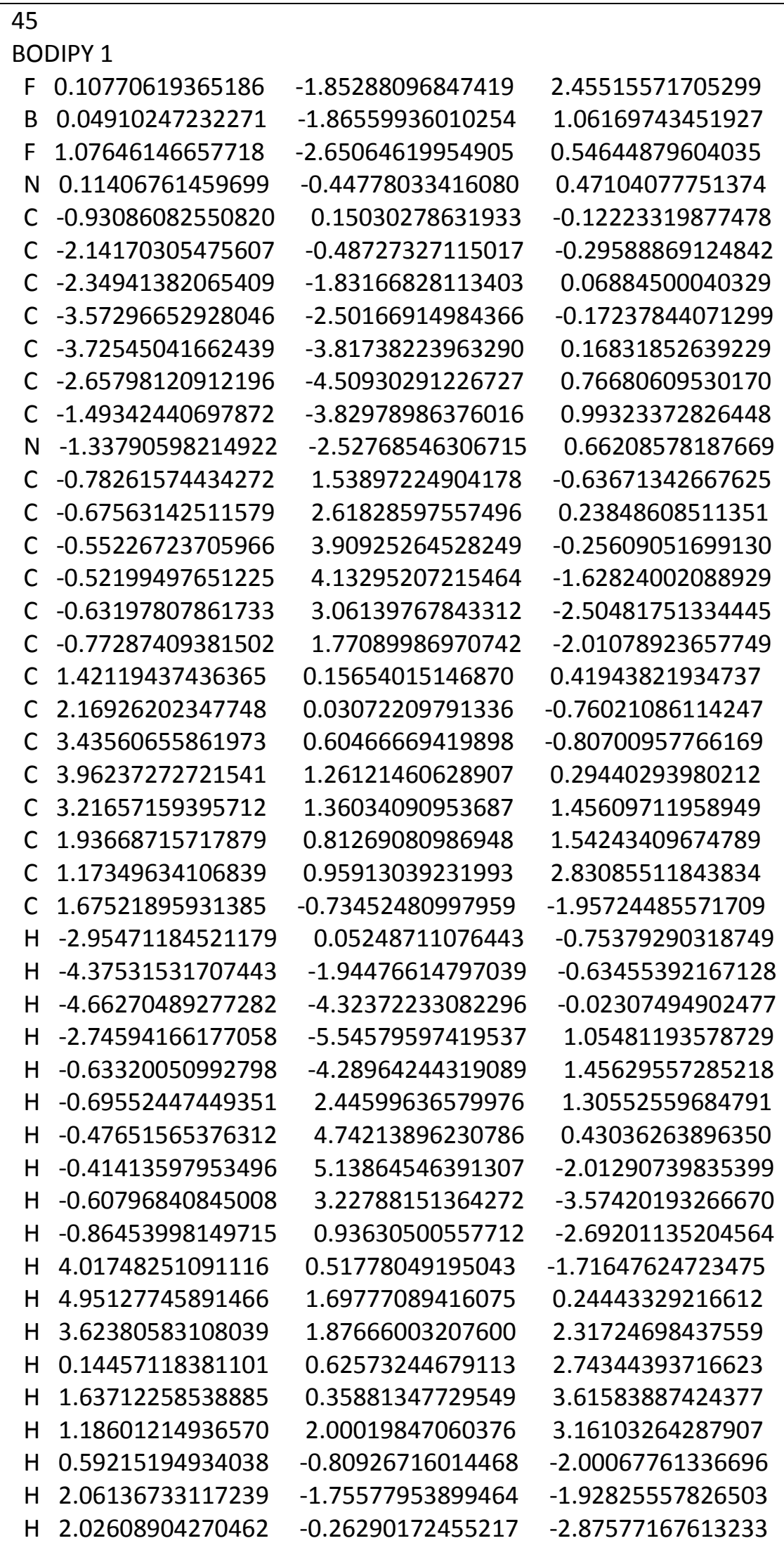




\begin{tabular}{|c|c|c|c|}
\hline 51 & & & \\
\hline & DIPY 2 & & \\
\hline $\mathrm{F}$ & 0.51130865936146 & 2.38698626223856 & 0.24885278524996 \\
\hline B & 0.65095545049293 & 1.23812123369622 & -0.52296324625539 \\
\hline $\mathrm{F}$ & 0.51448414125646 & 1.55546005172838 & -1.87686131540772 \\
\hline $\mathrm{N}$ & -0.43422584761233 & 0.20648331325870 & -0.08872568838318 \\
\hline $\mathrm{C}$ & -0.20857713882707 & -1.11128251027976 & -0.13392711166370 \\
\hline $\mathrm{C}$ & 1.07528315220574 & -1.58927216288774 & -0.32383065328930 \\
\hline $\mathrm{C}$ & 2.20841888326510 & -0.76435097843351 & -0.34518748097800 \\
\hline $\mathrm{C}$ & 3.49481283091436 & -1.38450946603039 & -0.32782711594208 \\
\hline C & 4.61955723081505 & -0.64032449644618 & -0.30633172889949 \\
\hline $\mathrm{C}$ & 4.53188314231213 & 0.77899374346929 & -0.32520749575386 \\
\hline $\mathrm{C}$ & 5.68956084813035 & 1.57594499447476 & -0.33493176594789 \\
\hline $\mathrm{C}$ & 5.60249142394342 & 2.94556627226281 & -0.39345487663223 \\
\hline $\mathrm{C}$ & 4.33732946728425 & 3.54144647537144 & -0.45683854490493 \\
\hline C & 3.18239622343473 & 2.78723104807636 & -0.44620928340220 \\
\hline $\mathrm{C}$ & 3.24800128486733 & 1.38313907803612 & -0.36531105796351 \\
\hline $\mathrm{N}$ & 2.09988721664918 & 0.58941073999191 & -0.34844831876897 \\
\hline $\mathrm{C}$ & -1.28851460675704 & -2.12183712721711 & 0.03975276314863 \\
\hline $\mathrm{C}$ & -1.42127819474518 & -3.12066248525509 & -0.92916155287747 \\
\hline $\mathrm{C}$ & -2.37011073959904 & -4.12440832764723 & -0.78729679271289 \\
\hline $\mathrm{C}$ & -3.18936835933153 & -4.15331698039953 & 0.33469990094578 \\
\hline C & -3.06104342935064 & -3.16723349638231 & 1.30563180817916 \\
\hline $\mathrm{C}$ & -2.12110932316429 & -2.15668947031793 & 1.16004267724100 \\
\hline $\mathrm{C}$ & -1.76043461238770 & 0.69754117330242 & 0.18928168872282 \\
\hline $\mathrm{C}$ & -2.73034013037250 & 0.68859391313216 & -0.81788031867860 \\
\hline $\mathrm{C}$ & -4.02224397679150 & 1.10008495196943 & -0.48899541602695 \\
\hline $\mathrm{C}$ & -4.33994724847676 & 1.50677670934629 & 0.79534308777556 \\
\hline $\mathrm{C}$ & -3.35675282428645 & 1.52869965074679 & 1.77414053650811 \\
\hline $\mathrm{C}$ & -2.05317050751949 & 1.13574243777960 & 1.48829014644703 \\
\hline C & -1.01182106872843 & 1.15179738864744 & 2.57165787244310 \\
\hline $\mathrm{C}$ & -2.43463415069673 & 0.21997448067489 & -2.21600625357599 \\
\hline $\mathrm{H}$ & 1.23397611268048 & -2.65492228173262 & -0.32848038272277 \\
\hline $\mathrm{H}$ & 3.52918207277643 & -2.46491985562390 & -0.31981839531139 \\
\hline $\mathrm{H}$ & 5.59671876910425 & -1.10715585412725 & -0.28373645444545 \\
\hline $\mathrm{H}$ & 6.65255038530559 & 1.08181836934146 & -0.30067980794449 \\
\hline $\mathrm{H}$ & 6.49600123885617 & 3.55474987656998 & -0.40163699894238 \\
\hline $\mathrm{H}$ & 4.25577882547702 & 4.61890214811982 & -0.52129949927942 \\
\hline $\mathrm{H}$ & 2.22694130125057 & 3.27771917667641 & -0.49919085195116 \\
\hline $\mathrm{H}$ & -0.78408902118720 & -3.09928691298477 & -1.80306677687098 \\
\hline $\mathrm{H}$ & -2.46684858169290 & -4.88491483419423 & -1.55146502986392 \\
\hline $\mathrm{H}$ & -3.92372714241831 & -4.93934578697587 & 0.45392835998635 \\
\hline $\mathrm{H}$ & -3.69114481914676 & -3.18558149300889 & 2.18508064374863 \\
\hline $\mathrm{H}$ & -2.03353091991959 & -1.40131827293019 & 1.92507390838594 \\
\hline $\mathrm{H}$ & -4.78399749011565 & 1.09423968064450 & -1.25883698088614 \\
\hline $\mathrm{H}$ & -5.34886308579391 & 1.81658323663526 & 1.03521142855619 \\
\hline $\mathrm{H}$ & -3.59970268638991 & 1.85259877746809 & 2.77836613194336 \\
\hline $\mathrm{H}$ & -1.46926866380595 & 1.39244157383724 & 3.53071371823689 \\
\hline $\mathrm{H}$ & -0.50555743313809 & 0.18849079858625 & 2.66446567364747 \\
\hline $\mathrm{H}$ & -0.24077122207708 & 1.88994984163104 & 2.35701005264159 \\
\hline $\mathrm{H}$ & -3.14500901416669 & 0.65962085473736 & -2.91500307713788 \\
\hline $\mathrm{H}$ & -2.53109004480619 & -0.86707075554544 & -2.28940467696955 \\
\hline $\mathrm{H}$ & -1.42994237707810 & 0.49339729596894 & -2.52952823341771 \\
\hline
\end{tabular}




\begin{tabular}{|c|c|c|c|}
\hline \\
\hline \multicolumn{4}{|c|}{ BODIPY 3} \\
\hline & 0.55398198217550 & -2.12774873471710 & 0.56522501725151 \\
\hline B & 0.90444719216213 & -1.14677316845211 & -0.36703510302255 \\
\hline $\mathrm{F}$ & 0.75649300524201 & -1.64616788836903 & -1.65986306071700 \\
\hline N & -0.02157678877241 & 0.08127747738218 & -0.15046102403576 \\
\hline C & 0.42635446702100 & 1.27511818266801 & 0.25139961694116 \\
\hline C & 1.79232757334599 & 1.51637604914751 & 0.30539810971185 \\
\hline C & 2.76527564436249 & 0.53024189218197 & 0.10648816981376 \\
\hline C & 4.14593868183410 & 0.88287571095073 & 0.22879443960002 \\
\hline C & 5.10940803629763 & -0.05793824235282 & 0.13751428038939 \\
\hline C & 4.76071058301467 & -1.41772025191007 & -0.09494855477798 \\
\hline C & 5.73776964468656 & -2.42629911453811 & -0.16188916578489 \\
\hline C & 5.38294645999077 & -3.73521108897906 & -0.38133258256451 \\
\hline C & 4.03038151437091 & -4.05580151161351 & -0.55150105733900 \\
\hline C & 3.05015053549191 & -3.08888640814806 & -0.49731107030097 \\
\hline C & 3.39154236702374 & -1.74597664405832 & -0.25513511922374 \\
\hline $\mathrm{N}$ & 2.41921809368914 & -0.75240705090721 & -0.16012270899854 \\
\hline C & -0.47317489582015 & 2.36423264565270 & 0.72187870939671 \\
\hline C & -1.66588214649441 & 2.73491862124782 & 0.09700495833798 \\
\hline C & -2.43812796436766 & 3.76937439011039 & 0.60514258438588 \\
\hline C & -2.05060975466121 & 4.44343801956979 & 1.75621977246556 \\
\hline C & -0.87128492863147 & 4.08004648162624 & 2.39402138644105 \\
\hline C & -0.09409984028751 & 3.05265321148817 & 1.88082227345912 \\
\hline C & -1.42858325918629 & -0.20102433900468 & -0.29828423926965 \\
\hline C & -1.99362010291205 & -0.12145591842522 & -1.58251171077117 \\
\hline C & -3.35303012106434 & -0.39541688561744 & -1.71374181972586 \\
\hline C & -4.12650471646001 & -0.73628143163481 & -0.61516260225740 \\
\hline C & -3.54300419617566 & -0.82663530759631 & 0.63715122859897 \\
\hline C & -2.18602105273017 & -0.56958674592531 & 0.82199935907859 \\
\hline C & -1.58409898209213 & -0.72081199920351 & 2.20666545882113 \\
\hline C & -1.74874478010142 & -2.15717259308814 & 2.72188685236966 \\
\hline C & -2.17196095338642 & 0.28332238344140 & 3.20302836658398 \\
\hline C & -1.18287967616259 & 0.23834781436455 & -2.81821255026419 \\
\hline C & -1.23297847215303 & -0.89805487454796 & -3.84693394210545 \\
\hline C & -1.61995898394133 & 1.56493709819199 & -3.45593137731867 \\
\hline $\mathrm{H}$ & 2.13509649647381 & 2.50831460110570 & 0.54492366993735 \\
\hline $\mathrm{H}$ & 4.38363097767561 & 1.92000813511739 & 0.41742878594761 \\
\hline $\mathrm{H}$ & 6.15470577009020 & 0.20519250656049 & 0.24493784913230 \\
\hline $\mathrm{H}$ & 6.77606228568799 & -2.14761020220335 & -0.03074629722239 \\
\hline $\mathrm{H}$ & 6.13668738658056 & -4.50963995952908 & -0.42437646809851 \\
\hline $\mathrm{H}$ & 3.74071335909140 & -5.08301012496888 & -0.73056016223490 \\
\hline $\mathrm{H}$ & 2.01984817798479 & -3.36751123767137 & -0.63234899380933 \\
\hline $\mathrm{H}$ & -1.99794232173598 & 2.22295905202295 & -0.78757416114157 \\
\hline $\mathrm{H}$ & -3.35317228316223 & 4.04476468110161 & 0.09789560973763 \\
\hline $\mathrm{H}$ & -2.66419469811105 & 5.24059654011531 & 2.15491357706229 \\
\hline $\mathrm{H}$ & -0.55941602628761 & 4.58498775616703 & 3.29846602168789 \\
\hline $\mathrm{H}$ & 0.80498824120510 & 2.75197294748139 & 2.39960976943380 \\
\hline $\mathrm{H}$ & -3.81327816635130 & -0.34253955206640 & -2.69192534378946 \\
\hline $\mathrm{H}$ & -5.18040359379608 & -0.95094663148857 & -0.73983930812508 \\
\hline $\mathrm{H}$ & -4.14702009878842 & -1.11316398330185 & 1.48784894962373 \\
\hline $\mathrm{H}$ & -0.51685679445829 & -0.52723909183296 & 2.12897406151020 \\
\hline $\mathrm{H}$ & -1.20622709890908 & -2.28268391090759 & 3.66106745299100 \\
\hline
\end{tabular}




\begin{tabular}{|llll|}
\hline H & -2.79822339092913 & -2.39318686754874 & 2.91087500615564 \\
H & -1.35558361213256 & -2.87252387739991 & 2.00250664579839 \\
H & -2.02426734329913 & 1.30949821757597 & 2.86853945461412 \\
H & -3.24359691706078 & 0.12339873016251 & 3.34088040093919 \\
H & -1.68967941248878 & 0.16841491379503 & 4.17617554424230 \\
H & -0.14582652008992 & 0.35127369734016 & -2.51541612165569 \\
H & -2.23701160927824 & -1.01597058664134 & -4.25953051140511 \\
H & -0.55552459080328 & -0.68610622139927 & -4.67606895744117 \\
H & -0.93239928587498 & -1.83993476899157 & -3.39362597250905 \\
H & -1.05291182285489 & 1.73995567453700 & -4.37191334190587 \\
H & -2.68024138268890 & 1.55792303861738 & -3.71741156520430 \\
H & -1.43904989099715 & 2.41236674531629 & -2.79375848944005 \\
\hline
\end{tabular}




\begin{tabular}{|cccc|}
\hline 33 & & & \\
BODIPY 4 & & \\
F & 0.98948702500250 & 2.15108817988283 & 1.25803937407457 \\
B & 0.44044584112869 & 1.58097845347964 & 0.11279005637824 \\
F & 0.33463078655833 & 2.53645133523594 & -0.89262072994246 \\
N & 1.31724923060721 & 0.38951380676446 & -0.36765815014273 \\
C & 0.99293495145190 & -0.96132386562950 & -0.24219047659465 \\
C & -0.23929509873660 & -1.33841919639307 & 0.26792836435883 \\
C & -1.20728583684771 & -0.40063672278158 & 0.59652315288989 \\
C & -2.55809686727021 & -0.59830995591144 & 1.02655983466904 \\
C & -3.22206116417669 & -1.90868452689314 & 1.28762489864332 \\
C & -3.09969974986791 & 0.67652725117315 & 1.09093681919322 \\
C & -2.09525140483419 & 1.61167324532541 & 0.71680980149672 \\
C & -2.53312455525670 & 2.95935855058779 & 0.72958961696557 \\
C & -3.84183373087056 & 3.02978266274622 & 1.10359551559764 \\
S & -4.58998865990519 & 1.49277762386898 & 1.45777156757521 \\
N & -0.95391785929432 & 0.96051681526369 & 0.42838275555374 \\
C & 2.06381475795863 & -1.76657350326770 & -0.74676027612327 \\
C & 2.09042965198677 & -3.25366689372340 & -0.83681656840487 \\
C & 3.02069445838423 & -0.86012357180886 & -1.17164394038510 \\
C & 2.54055525146291 & 0.45474639446822 & -0.91832395638430 \\
C & 3.44471745315666 & 1.47805497022323 & -1.29713228500317 \\
C & 4.57101300461481 & 0.93508001781414 & -1.83920819829456 \\
S & 4.58823669083988 & -0.80847938644096 & -1.91507240847202 \\
H & -0.46416040327400 & -2.38983205059013 & 0.38447695097104 \\
H & -3.64666497081549 & -2.32926804723622 & 0.37136564397553 \\
H & -2.52418885179667 & -2.63938109258069 & 1.69721521987757 \\
H & -4.03943000084136 & -1.79265271315098 & 1.99946051564380 \\
H & -1.92478248239823 & 3.80896454671125 & 0.46429462498505 \\
H & -4.44108083862210 & 3.92201213377367 & 1.19083884560114 \\
H & 1.38285028810489 & -3.61901671313695 & -1.58474995206370 \\
H & 1.82765297397063 & -3.72034029161731 & 0.11456056924239 \\
H 3.08046485136051 & -3.60622883201929 & -1.12387182396650 \\
H & 3.26729596629835 & 2.53490306355114 & -1.17713930735161 \\
H & 5.42829029192103 & 1.47051231231144 & -2.21467705456357 \\
\hline
\end{tabular}




\begin{tabular}{|llll|}
\hline BO & & & \\
BODIPY 5 & & \\
F & 1.08229686135815 & 1.25356458567903 & 1.73275383582622 \\
B & 0.52524662551724 & 1.10638810108515 & 0.46646402965977 \\
F & 0.41336051056291 & 2.34805929087109 & -0.15626200429651 \\
N & 1.38280764510931 & 0.13499093335468 & -0.39458758677515 \\
C & 0.89854832399599 & -0.96603923298262 & -1.09421023672653 \\
C & -0.43146681357033 & -1.35548639580995 & -0.96103220361675 \\
C & -1.30438199366779 & -0.67560008912692 & -0.14182997973022 \\
C & -2.67793557444020 & -0.91285600094000 & 0.15673155161170 \\
C & -3.05898028892694 & 0.08752540693638 & 1.03127213818938 \\
C & -1.93606270249467 & 0.91819010776358 & 1.26568875514508 \\
N & -0.89209989276109 & 0.45326974852197 & 0.56816547222421 \\
C & -1.84397747992563 & 2.12422102306458 & 2.12679063135720 \\
C & -3.49958307779453 & -2.04272312998255 & -0.36383194031358 \\
C & 1.95011154360509 & -1.57091936900831 & -1.82672169448748 \\
C & 3.08071767255849 & -0.82001862133390 & -1.54849407955573 \\
C & 2.70410346818631 & 0.21983199391633 & -0.65577723247087 \\
C & 3.78318395175038 & 1.04633239316702 & -0.25350786350844 \\
C & 4.94103424070006 & 0.63176718955671 & -0.84045637749634 \\
S & 4.77519153508748 & -0.74813844274162 & -1.89633409430220 \\
H & -0.77770933758430 & -2.22344922840516 & -1.50561325719978 \\
H & -4.03221164008707 & 0.22671687652456 & 1.47354013248569 \\
H & -1.43664220495180 & 2.96340950035749 & 1.56126350695701 \\
H & -1.15963936678148 & 1.94344984620936 & 2.95817103943384 \\
H & -2.82160483585626 & 2.39096242461542 & 2.52292354020463 \\
H & -3.50012632969011 & -2.07246935391549 & -1.45604071721098 \\
H & -4.53178951153348 & -1.95550223440122 & -0.02767458934845 \\
H & -3.11405204879620 & -3.00302844148489 & -0.01166784083772 \\
H & 1.85371102380169 & -2.44562487949444 & -2.44783139547915 \\
H & 3.70818059473803 & 1.87747637310980 & 0.42927666595421 \\
H & 5.91956710189073 & 1.06579862489390 & -0.71086820569308 \\
\hline
\end{tabular}




\begin{tabular}{|c|c|c|c|}
\hline \\
\hline \multicolumn{4}{|c|}{ BODIPY 5 , fully optimized $1^{\text {st }}$ ES. } \\
\hline $\mathrm{t}$ & 1.13602946568688 & $1.001364208163 / 7$ & 1.78939103902416 \\
\hline B & 0.53917589542901 & 0.98447316780177 & 0.53333662706533 \\
\hline $\mathrm{F}$ & 0.56081316401232 & 2.26009461608749 & -0.02264644315581 \\
\hline $\mathrm{N}$ & 1.39264647038598 & 0.04120871409006 & -0.38705953765955 \\
\hline C & 0.92038730335965 & -1.07818597893406 & -1.08346144953449 \\
\hline $\mathrm{C}$ & -0.46400023159620 & -1.45592980522651 & -0.93639149947568 \\
\hline C & -1.30401966982550 & -0.72168671247606 & -0.13628069369559 \\
\hline C & -2.69600003448466 & -0.89743275221653 & 0.14849965111911 \\
\hline C & -3.05718664866266 & 0.13651843495844 & 1.00330493465309 \\
\hline C & -1.92544793426166 & 0.92936705997604 & 1.25090195064798 \\
\hline $\mathrm{N}$ & -0.87140917585699 & 0.40747375130428 & 0.56862016796529 \\
\hline C & -1.80992980661153 & 2.14997662555045 & 2.08861141632065 \\
\hline $\mathrm{C}$ & -3.54210100078676 & -2.00422295105311 & -0.37285362710763 \\
\hline C & 1.95328220134300 & -1.62331675911445 & -1.80869982717784 \\
\hline C & 3.11011956767269 & -0.79016605826874 & -1.53900051474843 \\
\hline $\mathrm{C}$ & 2.70642095014894 & 0.20459392409135 & -0.65892384068636 \\
\hline C & 3.74413119484930 & 1.10549843363018 & -0.25744975784002 \\
\hline C & 4.93050250279512 & 0.77184858773431 & -0.84620247320676 \\
\hline $\mathrm{S}$ & 4.75742605671271 & -0.61877304206264 & -1.88430450675111 \\
\hline $\mathrm{H}$ & -0.83126012143222 & -2.31581321383276 & -1.47257672579028 \\
\hline $\mathrm{H}$ & -4.03625409858970 & 0.31665945427234 & 1.41826069562138 \\
\hline $\mathrm{H}$ & -1.43761489437393 & 2.99102980364836 & 1.49866767541716 \\
\hline $\mathrm{H}$ & -1.10060666534383 & 2.00587763185862 & 2.90761649269048 \\
\hline $\mathrm{H}$ & -2.77925018312001 & 2.41678107244408 & 2.50553517757358 \\
\hline $\mathrm{H}$ & -3.63163091234460 & -1.97249203517246 & -1.46328342942855 \\
\hline $\mathrm{H}$ & -4.54602644149692 & -1.95358071273445 & 0.04760390071346 \\
\hline $\mathrm{H}$ & -3.11989506998755 & -2.98020902161473 & -0.11501092998251 \\
\hline $\mathrm{H}$ & 1.90053271498399 & -2.49052760088154 & -2.44385059229599 \\
\hline $\mathrm{H}$ & 3.61038798813863 & 1.93034678820159 & 0.42518625992536 \\
\hline $\mathrm{H}$ & 5.89057541325650 & 1.24932336977490 & -0.75724014020046 \\
\hline
\end{tabular}




\begin{tabular}{|c|c|c|c|}
\hline \multicolumn{4}{|l|}{44} \\
\hline \multicolumn{4}{|c|}{ BODIPY 6} \\
\hline $\mathrm{F}$ & 2.42369064389810 & 1.10050922195114 & -1.34477554078423 \\
\hline B & 1.86275719156445 & 0.67871628818758 & -0.15386550420753 \\
\hline & 2.10493297811750 & 1.60622375204439 & 0.86122248679092 \\
\hline $\mathrm{N}$ & 2.47332749383012 & -0.69125566311727 & 0.25605195440558 \\
\hline $\mathrm{C}$ & 1.75406236045318 & -1.81775181021828 & 0.62996481078303 \\
\hline $\mathrm{C}$ & 0.36788931939746 & -1.79804837960101 & 0.58660743607439 \\
\hline $\mathrm{C}$ & -0.33110296380295 & -0.69096661922158 & 0.15201397749774 \\
\hline $\mathrm{C}$ & -1.73282237541038 & -0.56226740052969 & -0.07655409078677 \\
\hline $\mathrm{C}$ & -1.90674658088962 & 0.65060843044170 & -0.71410094996729 \\
\hline $\mathrm{C}$ & -0.63845638694287 & 1.27794886529808 & -0.82926861519494 \\
\hline $\mathrm{C}$ & -0.42250766178095 & 2.60481884903473 & -1.40246599167132 \\
\hline $\mathrm{C}$ & 0.54271415898938 & 3.49590014212345 & -0.91790597282611 \\
\hline $\mathrm{C}$ & 0.67544295535549 & 4.75619532684824 & -1.47917527175543 \\
\hline $\mathrm{C}$ & -0.13998730634305 & 5.15065872610327 & -2.53317952909926 \\
\hline $\mathrm{C}$ & -1.10675495986067 & 4.27715229164583 & -3.02015591895186 \\
\hline $\mathrm{C}$ & -1.25019037857963 & 3.01893070075594 & -2.45748170833853 \\
\hline $\mathrm{N}$ & 0.30650755987711 & 0.46278671003487 & -0.30144986943358 \\
\hline $\mathrm{C}$ & -2.74431169126046 & -1.53949401237443 & 0.33421176417567 \\
\hline C & -3.71302250275573 & -2.00254697764470 & -0.56189817204734 \\
\hline $\mathrm{C}$ & -4.64946363101380 & -2.94802977855243 & -0.16481936803807 \\
\hline $\mathrm{C}$ & -4.64334026251988 & -3.43665683173193 & 1.13699743319750 \\
\hline $\mathrm{C}$ & -3.69643266248836 & -2.97044451967797 & 2.04161713737666 \\
\hline $\mathrm{C}$ & -2.75281296459243 & -2.03332451244743 & 1.64369903336813 \\
\hline $\mathrm{C}$ & 2.64181885895445 & -2.85874051947508 & 0.99925961827784 \\
\hline $\mathrm{C}$ & 3.91409875001733 & -2.33524444146895 & 0.84692971519459 \\
\hline $\mathrm{C}$ & 3.78256673587597 & -0.99577914461147 & 0.38462164347147 \\
\hline $\mathrm{C}$ & 5.02343391979242 & -0.34099221038831 & 0.18114404712654 \\
\hline $\mathrm{C}$ & 6.05680961261971 & -1.17553393712327 & 0.48580384055053 \\
\hline$S$ & 5.58152028065906 & -2.76478321988784 & 1.02981046370415 \\
\hline $\mathrm{H}$ & -0.18401043702091 & -2.68207181988095 & 0.87109474233593 \\
\hline $\mathrm{H}$ & -2.84339254728531 & 1.09676244492077 & -1.00102072588202 \\
\hline $\mathrm{H}$ & 1.17457370236911 & 3.20988776435490 & -0.09336721215845 \\
\hline $\mathrm{H}$ & 1.42127781853368 & 5.43525264327310 & -1.08718177531838 \\
\hline $\mathrm{H}$ & -0.02287433042419 & 6.13279923351979 & -2.97204080858864 \\
\hline $\mathrm{H}$ & -1.74447546330759 & 4.57477288461387 & -3.84229828757546 \\
\hline $\mathrm{H}$ & -1.98972023665914 & 2.33610814085899 & -2.85408800255396 \\
\hline $\mathrm{H}$ & -3.70964596788557 & -1.63494404646630 & -1.57951244956533 \\
\hline $\mathrm{H}$ & -5.38051744991748 & -3.30955801650495 & -0.87614271311299 \\
\hline $\mathrm{H}$ & -5.37386014829930 & -4.17264347192113 & 1.44641103940972 \\
\hline $\mathrm{H}$ & -3.69456180147474 & -3.33532935670456 & 3.06040857529192 \\
\hline $\mathrm{H}$ & -2.03021884880555 & -1.65490258337327 & 2.35476452561648 \\
\hline $\mathrm{H}$ & 2.34508247128292 & -3.84144013920887 & 1.32456626593061 \\
\hline $\mathrm{H}$ & 5.14060939216747 & 0.66963946737705 & -0.17565489568524 \\
\hline $\mathrm{H}$ & 7.10821235556567 & -0.94332247125603 & 0.41960286296332 \\
\hline
\end{tabular}




\begin{tabular}{|c|c|c|c|}
\hline 42 & & & \\
\hline & DIPY 7 & & \\
\hline $\mathrm{F}$ & 1.25126198486302 & 0.61841629337150 & 1.91444235569150 \\
\hline B & 1.54191004081261 & 0.42069829354856 & 0.56214025641018 \\
\hline $\mathrm{F}$ & 1.08308258556376 & 1.49752942329480 & -0.18636320166418 \\
\hline $\mathrm{N}$ & 3.06946152984215 & 0.26798499879316 & 0.35622480172317 \\
\hline $\mathrm{C}$ & 3.74361099118238 & -0.93762199053921 & 0.22124921805825 \\
\hline $\mathrm{C}$ & 3.01833917355586 & -2.11035058090169 & 0.02309922985500 \\
\hline $\mathrm{C}$ & 1.64397744023978 & -2.10268006083697 & -0.07556985209584 \\
\hline $\mathrm{C}$ & 0.76066229911540 & -3.14961250771418 & -0.45547254271004 \\
\hline $\mathrm{C}$ & -0.49984472980570 & -2.61551766510390 & -0.52874023982871 \\
\hline $\mathrm{C}$ & -1.82149755818587 & -3.20457551133539 & -0.89532638635504 \\
\hline $\mathrm{C}$ & -2.68300367565034 & -2.12227301564529 & -1.55565681687143 \\
\hline $\mathrm{C}$ & -2.73596365752573 & -0.85932817019084 & -0.73038701238276 \\
\hline $\mathrm{C}$ & -3.89571017032417 & -0.11413472177386 & -0.66869454729877 \\
\hline $\mathrm{C}$ & -3.94419370350506 & 1.08727958401409 & 0.04470381115892 \\
\hline 0 & -5.12036310339582 & 1.75680310180322 & -0.00223178997598 \\
\hline $\mathrm{C}$ & -5.20895585981190 & 3.01803083912424 & 0.64434417776925 \\
\hline $\mathrm{C}$ & -2.81726836005852 & 1.52135813377531 & 0.74286451158011 \\
\hline $\mathrm{C}$ & -1.65025936582999 & 0.77930270376990 & 0.68891509163914 \\
\hline $\mathrm{C}$ & -1.57431120300475 & -0.40370596121382 & -0.05391452418391 \\
\hline $\mathrm{C}$ & -0.39832115351752 & -1.23841699313395 & -0.14979550871203 \\
\hline $\mathrm{N}$ & 0.89315711687415 & -0.93584544795618 & 0.10048607409559 \\
\hline $\mathrm{C}$ & 5.13946406522412 & -0.70616182031362 & 0.17569269511511 \\
\hline C & 5.29376654315707 & 0.67086225902672 & 0.25935607729290 \\
\hline $\mathrm{C}$ & 4.00100337562932 & 1.24764983123554 & 0.37103630393892 \\
\hline $\mathrm{C}$ & 4.00885721463602 & 2.66317510571510 & 0.44106994706869 \\
\hline C & 5.28658550196950 & 3.13434665553626 & 0.37724844732438 \\
\hline $\mathrm{S}$ & 6.51343246324280 & 1.90111555251291 & 0.24004486525925 \\
\hline $\mathrm{H}$ & 3.54822384990013 & -3.04167708397140 & -0.12231297050493 \\
\hline $\mathrm{H}$ & 1.06562719940733 & -4.16099497756509 & -0.67418331201573 \\
\hline $\mathrm{H}$ & -1.69680036481803 & -4.05724791525366 & -1.56416591265794 \\
\hline $\mathrm{H}$ & -2.32802643487971 & -3.57460348754159 & 0.00265056908203 \\
\hline $\mathrm{H}$ & -3.69333063754540 & -2.48886758004974 & -1.73836251395513 \\
\hline $\mathrm{H}$ & -2.25022285024457 & -1.88225097671800 & -2.53351885876134 \\
\hline $\mathrm{H}$ & -4.78679833686533 & -0.43882371149815 & -1.18970940902039 \\
\hline $\mathrm{H}$ & -4.45303065162841 & 3.70920735070831 & 0.26406583639164 \\
\hline $\mathrm{H}$ & -6.19941347177858 & 3.40153199048629 & 0.41364138587288 \\
\hline $\mathrm{H}$ & -5.09852150185064 & 2.91863171318586 & 1.72779096628657 \\
\hline $\mathrm{H}$ & -2.83546077583505 & 2.42900833013122 & 1.32562493721480 \\
\hline $\mathrm{H}$ & -0.79482614540491 & 1.11801076964198 & 1.24880032050008 \\
\hline $\mathrm{H}$ & 5.89423584338962 & -1.46582270925980 & 0.05801311330837 \\
\hline $\mathrm{H}$ & 3.13060295452506 & 3.28331528519894 & 0.51814109696090 \\
\hline $\mathrm{H}$ & 5.59846153833588 & 4.16635467364242 & 0.40286030939652 \\
\hline
\end{tabular}




\begin{tabular}{|c|c|c|c|}
\hline 52 & & & \\
\hline & DIPY 8 & & \\
\hline $\mathrm{F}$ & 0.80947280095270 & 2.56972738938428 & -1.20221929811850 \\
\hline B & -0.01136814494250 & 2.35112114896990 & -0.10442367550752 \\
\hline $\mathrm{F}$ & 0.58033474603531 & 2.87889701893065 & 1.04696736346536 \\
\hline $\mathrm{N}$ & -1.38623612902282 & 3.02212152956882 & -0.33791564007393 \\
\hline $\mathrm{C}$ & -2.62135756078742 & 2.42735734824967 & -0.13192656496871 \\
\hline $\mathrm{C}$ & -2.69201217442503 & 1.07086154345677 & 0.17358907465167 \\
\hline $\mathrm{C}$ & -1.56447234252055 & 0.28459612463177 & 0.24963794168577 \\
\hline $\mathrm{C}$ & -1.45608941417019 & -1.12871362220772 & 0.45888330202356 \\
\hline C & -2.57175732484543 & -2.06061657973934 & 0.64575197928363 \\
\hline $\mathrm{C}$ & -2.53273782483940 & -3.01430812166900 & 1.66971261600941 \\
\hline $\mathrm{C}$ & -3.58549485246175 & -3.89759136636081 & 1.86088950722939 \\
\hline C & -4.69932463684684 & -3.84929127104853 & 1.03015573647076 \\
\hline $\mathrm{C}$ & -4.74624828802166 & -2.91645173748151 & 0.00004852592949 \\
\hline $\mathrm{C}$ & -3.69470252450718 & -2.03095846522801 & -0.19148922326458 \\
\hline $\mathrm{C}$ & -0.10713756125451 & -1.42435402708083 & 0.42504425941466 \\
\hline $\mathrm{C}$ & 0.62010712812279 & -2.72031773409847 & 0.54768139352091 \\
\hline $\mathrm{C}$ & 1.85157796631050 & -2.70406341726541 & -0.36126006199407 \\
\hline C & 2.66422758400437 & -1.43579105052187 & -0.23957327833363 \\
\hline $\mathrm{C}$ & 4.03268532879816 & -1.48305021426464 & -0.48162924774643 \\
\hline $\mathrm{C}$ & 4.80103335136116 & -0.32024032477738 & -0.45099115203003 \\
\hline 0 & 6.12723836776467 & -0.27255850650172 & -0.71928052251061 \\
\hline $\mathrm{C}$ & 6.80165556559069 & -1.47479511973429 & -1.05209172644095 \\
\hline $\mathrm{C}$ & 4.19809975972792 & 0.89818412474605 & -0.12788352133344 \\
\hline $\mathrm{C}$ & 2.84640520072534 & 0.95358566005563 & 0.11712776780195 \\
\hline $\mathrm{C}$ & 2.04246026966053 & -0.19765208385927 & 0.03635518063987 \\
\hline $\mathrm{C}$ & 0.60166473802027 & -0.20859614085546 & 0.19162492071338 \\
\hline $\mathrm{N}$ & -0.27984457001675 & 0.81343580486190 & 0.09522909203418 \\
\hline $\mathrm{C}$ & -3.65344779798719 & 3.37046888589796 & -0.34760867864104 \\
\hline $\mathrm{C}$ & -3.01297038491302 & 4.55105520729294 & -0.69800018941651 \\
\hline $\mathrm{C}$ & -1.61388080437340 & 4.31053594667197 & -0.67938791404198 \\
\hline $\mathrm{C}$ & -0.83258958688048 & 5.44567352682214 & -1.01148656448740 \\
\hline C & -1.63736116258158 & 6.51296523303992 & -1.27939304804366 \\
\hline $\mathrm{S}$ & -3.34725969149964 & 6.18738962591112 & -1.15034575082910 \\
\hline $\mathrm{H}$ & -3.65931432379588 & 0.61827757788385 & 0.33283800053973 \\
\hline $\mathrm{H}$ & -1.67790138769210 & -3.04373343470474 & 2.33170347785956 \\
\hline $\mathrm{H}$ & -3.54055182488412 & -4.61852503242108 & 2.66687397019432 \\
\hline $\mathrm{H}$ & -5.52319223089338 & -4.53367053554414 & 1.18491838178993 \\
\hline $\mathrm{H}$ & -5.60390535474836 & -2.87817743967193 & -0.65937958597366 \\
\hline $\mathrm{H}$ & -3.72876041519100 & -1.32725186457726 & -1.01243579657255 \\
\hline $\mathrm{H}$ & -0.03417036182941 & -3.55431653473613 & 0.29139403518371 \\
\hline $\mathrm{H}$ & 0.94013940358354 & -2.87555121649919 & 1.58506574144301 \\
\hline $\mathrm{H}$ & 2.48837474190788 & -3.56446090536719 & -0.15115107120372 \\
\hline $\mathrm{H}$ & 1.51769779831954 & -2.80340872212973 & -1.40092449897855 \\
\hline $\mathrm{H}$ & 4.48467444130710 & -2.43641639413999 & -0.71269331668685 \\
\hline $\mathrm{H}$ & 7.82233026576505 & -1.18653724227967 & -1.28966755178788 \\
\hline $\mathrm{H}$ & 6.80179670272528 & -2.17359230034448 & -0.21088876145483 \\
\hline $\mathrm{H}$ & 6.34906956187847 & -1.95723435659409 & -1.92260276555152 \\
\hline $\mathrm{H}$ & 4.81091690875738 & 1.78758634370446 & -0.07964582601870 \\
\hline $\mathrm{H}$ & 2.40603288051019 & 1.89832429682311 & 0.38231757566496 \\
\hline $\mathrm{H}$ & -4.70836662314777 & 3.17041498182187 & -0.25937578516514 \\
\hline $\mathrm{H}$ & 0.24482734829508 & 5.46702328462744 & -1.05766459416363 \\
\hline
\end{tabular}




\begin{tabular}{|c|c|c|c|}
\hline 40 & & & \\
\hline & DIPY 9 & & \\
\hline $\mathrm{F}$ & -1.30652845648576 & -1.77421761514967 & 1.14282202124745 \\
\hline B & -1.29286156398516 & -0.38686897276962 & 1.01030986249056 \\
\hline $\mathrm{F}$ & -0.57721930879731 & 0.19706375664343 & 2.05108319875037 \\
\hline $\mathrm{N}$ & -0.67644161089787 & 0.00699736127405 & -0.36442876823991 \\
\hline $\mathrm{C}$ & -1.38753680382182 & 0.50295515490162 & -1.45214564738349 \\
\hline C & -2.73990755831392 & 0.79827368885258 & -1.33026185277677 \\
\hline C & -3.40982564843631 & 0.64606833118180 & -0.13422021396533 \\
\hline $\mathrm{C}$ & -4.74032208855257 & 0.99703728092148 & 0.23167313038056 \\
\hline C & -4.84769660779087 & 0.73031984343365 & 1.58496138581771 \\
\hline $\mathrm{C}$ & -3.60634235058795 & 0.22488531560413 & 2.03601030778585 \\
\hline $\mathrm{N}$ & -2.75413858076219 & 0.16757823834440 & 1.00142641856154 \\
\hline $\mathrm{C}$ & -3.23148431101560 & -0.15074846274186 & 3.42342134530124 \\
\hline $\mathrm{C}$ & -5.78852950389622 & 1.53449195902310 & -0.68151735015133 \\
\hline $\mathrm{C}$ & -0.51312662032739 & 0.68766799625375 & -2.55420899977163 \\
\hline $\mathrm{C}$ & 0.73862606778861 & 0.30414647611186 & -2.10968952816753 \\
\hline $\mathrm{C}$ & 0.60881818177417 & -0.12039653404231 & -0.75767180430090 \\
\hline C & 1.82033561113096 & -0.54053379194403 & -0.17395083852302 \\
\hline C & 2.86825370248229 & -0.43901298081965 & -1.05540382196646 \\
\hline $\mathrm{C}$ & 4.25956487588229 & -0.79502139894143 & -0.79838671108273 \\
\hline $\mathrm{C}$ & 4.55795297698118 & -1.78093823857362 & 0.15302116645768 \\
\hline C & 5.87072444428135 & -2.11857807529163 & 0.43678562266304 \\
\hline $\mathrm{C}$ & 6.91681486881759 & -1.48236380251718 & -0.22419599474530 \\
\hline $\mathrm{C}$ & 6.63521126498698 & -0.51035786850171 & -1.17844635347173 \\
\hline $\mathrm{C}$ & 5.32104136469635 & -0.16943049926470 & -1.46604294989649 \\
\hline $\mathrm{S}$ & 2.38267237383992 & 0.17820419068693 & -2.63852208732533 \\
\hline $\mathrm{H}$ & -3.26754047910417 & 1.19256011702115 & -2.18771210844998 \\
\hline $\mathrm{H}$ & -5.71237452508000 & 0.88941016837578 & 2.20907780189190 \\
\hline $\mathrm{H}$ & -2.41223828551949 & 0.47522771553349 & 3.77825900735962 \\
\hline $\mathrm{H}$ & -4.08562319451686 & -0.03078723923265 & 4.08718709451597 \\
\hline $\mathrm{H}$ & -2.87612643559222 & -1.18240328380355 & 3.46899722374426 \\
\hline $\mathrm{H}$ & -6.25225009526819 & 0.73586546070858 & -1.26761065879063 \\
\hline $\mathrm{H}$ & -6.57684007196749 & 2.03096402981965 & -0.11617321107051 \\
\hline $\mathrm{H}$ & -5.37404625130930 & 2.25608919297950 & -1.38780187513811 \\
\hline $\mathrm{H}$ & -0.80297573600210 & 1.07188346924967 & -3.51766468906607 \\
\hline $\mathrm{H}$ & 1.92381414122110 & -0.87183665390248 & 0.84656789611506 \\
\hline $\mathrm{H}$ & 3.75078290507429 & -2.29815693667820 & 0.65163137427515 \\
\hline $\mathrm{H}$ & 6.07902948005061 & -2.88716815560799 & 1.16951506844037 \\
\hline $\mathrm{H}$ & 7.94112655849777 & -1.74894167619332 & 0.00188265245385 \\
\hline $\mathrm{H}$ & 7.44247837068696 & -0.00903680049845 & -1.69574247993651 \\
\hline $\mathrm{H}$ & 5.11462789983835 & 0.60570723955347 & -2.19303563403244 \\
\hline
\end{tabular}




\begin{tabular}{|c|c|c|c|}
\hline 54 & & & \\
\hline & DIPY 10 & & \\
\hline $\mathrm{F}$ & 0.56875877978582 & -1.64568682750421 & -0.06497642160247 \\
\hline B & 0.16219941823744 & -0.42763801186903 & 0.45036416414210 \\
\hline $\mathrm{F}$ & 0.56573706786954 & -0.29608326745091 & 1.78400725704992 \\
\hline $\mathrm{N}$ & 0.77899417698526 & 0.71409423297657 & -0.39567944874483 \\
\hline $\mathrm{C}$ & 0.09773369566533 & 1.80620496221372 & -0.91119046018758 \\
\hline $\mathrm{C}$ & -1.27261303287925 & 1.91241233434201 & -0.72429777483211 \\
\hline $\mathrm{C}$ & -2.00242629058560 & 0.91898529423247 & -0.09171333542311 \\
\hline $\mathrm{C}$ & -3.41927293909102 & 0.76031300911508 & 0.02227344777822 \\
\hline C & -3.62415687360473 & -0.54705505840465 & 0.44363191777018 \\
\hline $\mathrm{C}$ & -2.36429259378714 & -1.14892207593519 & 0.64966187438273 \\
\hline $\mathrm{C}$ & -2.13499320555937 & -2.51799178135352 & 1.11368987476377 \\
\hline $\mathrm{C}$ & -2.90761120057254 & -3.55327858906038 & 0.57244815801353 \\
\hline $\mathrm{C}$ & -2.69540918915383 & -4.86834425377119 & 0.95996326056352 \\
\hline $\mathrm{C}$ & -1.72415789863940 & -5.16679892025740 & 1.91029616623312 \\
\hline $\mathrm{C}$ & -0.97615789519442 & -4.14062370667367 & 2.47578625362842 \\
\hline $\mathrm{C}$ & -1.17214210618505 & -2.82704590900056 & 2.07978896614397 \\
\hline $\mathrm{N}$ & -1.39349386315502 & -0.25780082630639 & 0.34244715009599 \\
\hline $\mathrm{C}$ & -4.44111463436509 & 1.78013667924909 & -0.19432328099212 \\
\hline $\mathrm{C}$ & -5.73394702754871 & 1.41685508722124 & -0.59397861014304 \\
\hline $\mathrm{C}$ & -6.72877370584277 & 2.37126913573353 & -0.73661255690608 \\
\hline C & -6.45451844917204 & 3.71273394063125 & -0.49343686174684 \\
\hline $\mathrm{C}$ & -5.17601497053584 & 4.09054664139771 & -0.10297943180374 \\
\hline $\mathrm{C}$ & -4.18232835811802 & 3.13500661540917 & 0.05058214171954 \\
\hline $\mathrm{C}$ & 1.00326224341306 & 2.63918992186878 & -1.62702277984364 \\
\hline $\mathrm{C}$ & 2.23770086940052 & 2.02486980730694 & -1.52470526404546 \\
\hline $\mathrm{C}$ & 2.06943563998947 & 0.83800719389748 & -0.75803277141523 \\
\hline $\mathrm{C}$ & 3.25237393534306 & 0.10690463749188 & -0.55573551463409 \\
\hline $\mathrm{C}$ & 4.33192530674885 & 0.71842077834838 & -1.14528855289011 \\
\hline $\mathrm{C}$ & 5.72221246647730 & 0.28570968873751 & -1.08176987070469 \\
\hline $\mathrm{C}$ & 6.72117317703821 & 0.87765115296091 & -1.86633245307213 \\
\hline $\mathrm{C}$ & 8.04365730666046 & 0.47230084483915 & -1.75645691396037 \\
\hline C & 8.39913020053014 & -0.53200792952798 & -0.86267835973253 \\
\hline $\mathrm{C}$ & 7.41646340019621 & -1.13614165347839 & -0.08495155921253 \\
\hline $\mathrm{C}$ & 6.09720861716187 & -0.73166228113963 & -0.18977957202551 \\
\hline $\mathrm{S}$ & 3.89421333566836 & 2.20969243679021 & -1.99682874525598 \\
\hline $\mathrm{H}$ & -1.79278372869649 & 2.76181434791100 & -1.13937936522019 \\
\hline $\mathrm{H}$ & -4.56792117064889 & -1.01143532370320 & 0.67299639806348 \\
\hline $\mathrm{H}$ & -3.64945216282147 & -3.32448681058764 & -0.18159054228476 \\
\hline $\mathrm{H}$ & -3.28188819830625 & -5.66069715287288 & 0.51395803646978 \\
\hline $\mathrm{H}$ & -1.55109240918963 & -6.19179700805145 & 2.21250131222468 \\
\hline $\mathrm{H}$ & -0.22796551544474 & -4.36457881798559 & 3.22476717207245 \\
\hline $\mathrm{H}$ & -0.57954662520136 & -2.03760012168890 & 2.51390548854878 \\
\hline $\mathrm{H}$ & -5.95145093934122 & 0.37790411830814 & -0.80320169806117 \\
\hline $\mathrm{H}$ & -7.72059534185763 & 2.06760786113509 & -1.04492249236699 \\
\hline $\mathrm{H}$ & -7.23353704262694 & 4.45574619172372 & -0.60167043978600 \\
\hline $\mathrm{H}$ & -4.95467503435782 & 5.12932876320595 & 0.10564341422784 \\
\hline $\mathrm{H}$ & -3.21107584201430 & 3.43588822998634 & 0.41588618164548 \\
\hline $\mathrm{H}$ & 0.74007585051591 & 3.55130790235002 & -2.13503157929315 \\
\hline $\mathrm{H}$ & 3.29854959683159 & -0.82333988576824 & -0.01486711931097 \\
\hline $\mathrm{H}$ & 6.46260897045435 & 1.65803081107021 & -2.57073746805318 \\
\hline $\mathrm{H}$ & 8.79979424475894 & 0.94344590143076 & -2.37024712266994 \\
\hline
\end{tabular}




\begin{tabular}{|llll|}
\hline H & 9.43074246302616 & -0.84620065897084 & -0.77237970595487 \\
H & 7.68074338005798 & -1.92039401664436 & 0.61245015624475 \\
H & 5.35061610168075 & -1.19466763387811 & 0.43854728039318
\end{tabular}




\begin{tabular}{|c|c|c|c|}
\hline 52 & & & \\
\hline & DIPY 11 & & \\
\hline $\mathrm{F}$ & 0.23116059455973 & 0.64956389302902 & 0.54948032653751 \\
\hline B & 0.19092093131448 & -0.09363199997632 & -0.62545448909033 \\
\hline $\mathrm{F}$ & -0.05813061682634 & -1.43673912997251 & -0.32844671233852 \\
\hline $\mathrm{N}$ & 1.54966862431597 & 0.01832270490850 & -1.38080018936566 \\
\hline $\mathrm{C}$ & 1.69086411624787 & 0.22745115360633 & -2.74685532627922 \\
\hline $\mathrm{C}$ & 0.59030052692825 & 0.59094241549703 & -3.50971401539872 \\
\hline $\mathrm{C}$ & -0.66061789575826 & 0.73794130437906 & -2.95211320392299 \\
\hline $\mathrm{C}$ & -1.84971444162617 & 1.21963566156963 & -3.55455986321673 \\
\hline C & -2.81983221791429 & 1.23393622784882 & -2.58751856490367 \\
\hline $\mathrm{C}$ & -4.25288353461500 & 1.64648570793923 & -2.64027703504370 \\
\hline $\mathrm{C}$ & -4.67802695935832 & 2.12004228721494 & -1.24922013388825 \\
\hline $\mathrm{C}$ & -4.32285539225536 & 1.10088581688396 & -0.19497417788159 \\
\hline $\mathrm{C}$ & -5.23613354598463 & 0.78395855033977 & 0.80074979302008 \\
\hline $\mathrm{C}$ & -4.90861102204600 & -0.13818936186110 & 1.79541250981597 \\
\hline 0 & -5.75601743964059 & -0.53543470424356 & 2.77549874612197 \\
\hline $\mathrm{C}$ & -7.09136919083947 & -0.05687785375403 & 2.76343083655202 \\
\hline $\mathrm{C}$ & -3.63765055032779 & -0.71685486706227 & 1.80695136467838 \\
\hline $\mathrm{C}$ & -2.72647457277881 & -0.41147503410193 & 0.82002811084877 \\
\hline $\mathrm{C}$ & -3.05302095596468 & 0.47926193813120 & -0.21724055017536 \\
\hline $\mathrm{C}$ & -2.23233991322794 & 0.72068652820977 & -1.38605217670004 \\
\hline $\mathrm{N}$ & -0.92366777262234 & 0.44692891845326 & -1.60992653079798 \\
\hline $\mathrm{C}$ & 3.05054182897384 & 0.10363684421351 & -3.12181390722837 \\
\hline $\mathrm{C}$ & 3.73334799237463 & -0.17086118265252 & -1.95141084797829 \\
\hline $\mathrm{C}$ & 2.79102160637165 & -0.21769143436993 & -0.88851260091640 \\
\hline $\mathrm{C}$ & 3.37383825332994 & -0.45199077773759 & 0.37433960807338 \\
\hline $\mathrm{C}$ & 4.74262815497635 & -0.57844914653545 & 0.28729563313677 \\
\hline $\mathrm{C}$ & 5.70698042406150 & -0.77239651747060 & 1.36220645807636 \\
\hline $\mathrm{C}$ & 5.28548971319237 & -0.98629922542839 & 2.68259695526389 \\
\hline $\mathrm{C}$ & 6.20672206138655 & -1.12409066756767 & 3.70762432270611 \\
\hline $\mathrm{C}$ & 7.57156423505496 & -1.06155740399500 & 3.44489821283903 \\
\hline $\mathrm{C}$ & 8.00595511723352 & -0.86695772549212 & 2.13911020604170 \\
\hline C & 7.08661898888530 & -0.72744994410794 & 1.11238528786696 \\
\hline $\mathrm{S}$ & 5.33649778747733 & -0.42950059085230 & -1.37017368205107 \\
\hline $\mathrm{H}$ & 0.72251254338947 & 0.80533718707755 & -4.56121101775666 \\
\hline $\mathrm{H}$ & -1.93992876199871 & 1.52988238719058 & -4.58371737037607 \\
\hline $\mathrm{H}$ & -4.40359676330168 & 2.43474920005571 & -3.37972155612478 \\
\hline $\mathrm{H}$ & -4.87861468876955 & 0.80002083764570 & -2.94570416476497 \\
\hline $\mathrm{H}$ & -5.74547911862470 & 2.33983829368166 & -1.22095589133689 \\
\hline $\mathrm{H}$ & -4.15136176032006 & 3.05432759710826 & -1.02413810041899 \\
\hline $\mathrm{H}$ & -6.20359872933719 & 1.26333731413102 & 0.78990433070181 \\
\hline $\mathrm{H}$ & -7.13060400432416 & 1.02607979636534 & 2.91496743059202 \\
\hline $\mathrm{H}$ & -7.59309588315733 & -0.31399248784033 & 1.82627356716033 \\
\hline $\mathrm{H}$ & -7.59345494332586 & -0.55484390737103 & 3.58830051772102 \\
\hline $\mathrm{H}$ & -3.39247710838123 & -1.42289162144147 & 2.58818441745236 \\
\hline $\mathrm{H}$ & -1.76722623241821 & -0.90083515748633 & 0.82594076488731 \\
\hline $\mathrm{H}$ & 3.43736402676890 & 0.22965242454755 & -4.11907165846099 \\
\hline $\mathrm{H}$ & 2.81772148706588 & -0.49311342127508 & 1.29608491511952 \\
\hline $\mathrm{H}$ & 4.22984742003593 & -1.04364529459049 & 2.90747511191844 \\
\hline $\mathrm{H}$ & 5.85626238911675 & -1.28389982737952 & 4.71877625863517 \\
\hline $\mathrm{H}$ & 8.28761974759234 & -1.16658780959031 & 4.24888564107997 \\
\hline $\mathrm{H}$ & 9.06454457327908 & -0.81945257088490 & 1.91775320219139 \\
\hline
\end{tabular}




\begin{tabular}{|c|c|c|c|}
\hline 62 & & & \\
\hline & DIPY 12 & & \\
\hline $\mathrm{F}$ & 0.95510243628582 & -0.77588434328879 & 1.71800914347833 \\
\hline B & 0.77891817949073 & -0.23073831459451 & 0.44280978143801 \\
\hline $\mathrm{F}$ & 1.08725325025070 & -1.16996286739910 & -0.53256181304912 \\
\hline $\mathrm{N}$ & 1.69373855841802 & 1.01184592793389 & 0.28493634745511 \\
\hline $\mathrm{C}$ & 1.27683252767375 & 2.32282953453775 & 0.46649568218442 \\
\hline $\mathrm{C}$ & -0.08127683644189 & 2.61234880776901 & 0.54144003647198 \\
\hline $\mathrm{C}$ & -1.04167944218488 & 1.63320054539219 & 0.40081561922310 \\
\hline $\mathrm{C}$ & -2.46368826058421 & 1.76267950650804 & 0.28805445231739 \\
\hline C & -3.23935516968984 & 3.00161611500506 & 0.38181790568174 \\
\hline $\mathrm{C}$ & -4.42156810589812 & 3.02799110052295 & 1.13283892990627 \\
\hline $\mathrm{C}$ & -5.16084494843379 & 4.19487479366137 & 1.25965186776635 \\
\hline $\mathrm{C}$ & -4.73590065112312 & 5.36324393110266 & 0.63777628117045 \\
\hline $\mathrm{C}$ & -3.57376969016489 & 5.34859972171845 & -0.12517819464096 \\
\hline $\mathrm{C}$ & -2.83771067506002 & 4.17963025726091 & -0.25920671278855 \\
\hline $\mathrm{C}$ & -2.95450355100742 & 0.48580325343163 & 0.09376065432290 \\
\hline $\mathrm{C}$ & -4.34123537305905 & 0.00266142546743 & -0.17128726858464 \\
\hline $\mathrm{C}$ & -4.26571059673930 & -1.23859156993774 & -1.06251870881968 \\
\hline $\mathrm{C}$ & -3.31653351524076 & -2.26113973440381 & -0.49315059889092 \\
\hline $\mathrm{C}$ & -3.65846840038371 & -3.60581764434128 & -0.50600170690799 \\
\hline $\mathrm{C}$ & -2.78391523519536 & -4.56425034577813 & 0.00460313680659 \\
\hline 0 & -3.06430015943930 & -5.88996599624147 & 0.06355774008580 \\
\hline $\mathrm{C}$ & -4.36751009558380 & -6.32239602534351 & -0.30200531265312 \\
\hline $\mathrm{C}$ & -1.54401605648823 & -4.16615731398115 & 0.51077466871336 \\
\hline $\mathrm{C}$ & -1.19826474725157 & -2.83164216623145 & 0.53019642097521 \\
\hline $\mathrm{C}$ & -2.07589854255383 & -1.84894139506307 & 0.04264204200275 \\
\hline $\mathrm{C}$ & -1.85261296733699 & -0.41796198018027 & 0.12683935850369 \\
\hline $\mathrm{N}$ & -0.70498608777345 & 0.28001734168876 & 0.29598046620598 \\
\hline $\mathrm{C}$ & 2.39569214760383 & 3.19345996655823 & 0.44052923635639 \\
\hline $\mathrm{C}$ & 3.49466675626619 & 2.37996797399639 & 0.22864602115459 \\
\hline $\mathrm{C}$ & 3.03905661294058 & 1.03689720944438 & 0.13955579503165 \\
\hline $\mathrm{C}$ & 4.06653346326897 & 0.09874023499998 & -0.08256603722084 \\
\hline C & 5.29756513296733 & 0.70716051887266 & -0.17468885849166 \\
\hline $\mathrm{C}$ & 6.59619874117981 & 0.08167583767975 & -0.39100754435172 \\
\hline $\mathrm{C}$ & 6.70391688447086 & -1.30788383017140 & -0.55104829509716 \\
\hline $\mathrm{C}$ & 7.93879369090765 & -1.90758687383904 & -0.73389112295816 \\
\hline $\mathrm{C}$ & 9.09880027291860 & -1.13954335568595 & -0.76748019082455 \\
\hline $\mathrm{C}$ & 9.00860296301619 & 0.23965051279494 & -0.62111502799109 \\
\hline C & 7.77396943934728 & 0.84200570954655 & -0.43614468816999 \\
\hline $\mathrm{S}$ & 5.20634138984336 & 2.46257258911273 & 0.01899946173490 \\
\hline $\mathrm{H}$ & -0.39184720856723 & 3.63772152260991 & 0.67325701270881 \\
\hline $\mathrm{H}$ & -4.74528581092976 & 2.13036770174665 & 1.64117997826371 \\
\hline $\mathrm{H}$ & -6.06670875990846 & 4.19413059498925 & 1.85156018529757 \\
\hline $\mathrm{H}$ & -5.30676369063104 & 6.27601359654853 & 0.74606735968404 \\
\hline $\mathrm{H}$ & -3.24111510557872 & 6.24822201690756 & -0.62660244662723 \\
\hline $\mathrm{H}$ & -1.95789395891122 & 4.17143108885912 & -0.88660321043850 \\
\hline $\mathrm{H}$ & -4.84276360106694 & -0.26100767001240 & 0.76763279779493 \\
\hline $\mathrm{H}$ & -4.93811292647557 & 0.78467295267599 & -0.64245736717542 \\
\hline $\mathrm{H}$ & -3.90946942527303 & -0.93470445415725 & -2.05340272593261 \\
\hline $\mathrm{H}$ & -5.25308518813800 & -1.68067375468589 & -1.19839586670274 \\
\hline $\mathrm{H}$ & -4.61284811099738 & -3.89456638449150 & -0.92088262774803 \\
\hline $\mathrm{H}$ & -5.13103643510063 & -5.79591050546789 & 0.27542498253290 \\
\hline
\end{tabular}




\begin{tabular}{|llll|}
\hline$H$ & -4.55449256835047 & -6.17535759784385 & -1.36954779227725 \\
$H$ & -4.40585701924643 & -7.38392659814871 & -0.07013538321360 \\
$H$ & -0.87695692291021 & -4.91962601309958 & 0.90571520287055 \\
$H$ & -0.25404385083577 & -2.54098407170134 & 0.96050131465482 \\
$H$ & 2.35692895644905 & 4.26475374083550 & 0.54713005095710 \\
$H$ & 3.90095555641979 & -0.96194677374703 & -0.18034148028170 \\
$H$ & 5.81547135360663 & -1.92242070301467 & -0.53079184908379 \\
$H$ & 7.99699618843132 & -2.98154177197372 & -0.85540822185609 \\
$H$ & 10.06161436290187 & -1.61190604040216 & -0.91024139179646 \\
H & 9.90179262684635 & 0.84999533885140 & -0.64823164264579 \\
$H$ & 7.72628719904967 & 1.91685572619700 & -0.31630584653202 \\
\hline
\end{tabular}




\begin{tabular}{|llll}
\hline 21 & & & \\
BODIPY 13 & & \\
B & 3.30920022130760 & 2.66923905311263 & 6.90690451841702 \\
N & 2.25765413552505 & 3.41934812569229 & 6.01132667850010 \\
N & 2.93362987920653 & 3.02845778289759 & 8.39052166237048 \\
F & 4.58547012447055 & 3.12385240658977 & 6.61953323151572 \\
F & 3.20920551517546 & 1.30200279479063 & 6.71044724980207 \\
C & 2.18575599910223 & 3.39689137078942 & 4.67796008451257 \\
H & 2.88905529740791 & 2.82094524944644 & 4.09733297101931 \\
C & 1.11553048283243 & 4.19548509756896 & 4.23175287069161 \\
H & 0.83365578679639 & 4.35128602780161 & 3.20390227818427 \\
C & 0.51605814695847 & 4.72326280520697 & 5.36069732884603 \\
H & -0.33720474999001 & 5.38099023363849 & 5.40725503105213 \\
C & 1.23415748902589 & 4.23541191996090 & 6.47828773300670 \\
C & 1.06079869290871 & 4.44460909681801 & 7.83774131807381 \\
H & 0.25449699952766 & 5.08493430335510 & 8.17202140172194 \\
C & 1.88836845865367 & 3.85672625107555 & 8.78176072488472 \\
C & 1.89008552851024 & 3.92919487600311 & 10.19528257269438 \\
H & 1.19376328387857 & 4.49786045019994 & 10.79071422644425 \\
C & 2.93761937199467 & 3.14309332179285 & 10.63994139694344 \\
H & 3.23897758308615 & 2.96352793450981 & 11.65831312367853 \\
C & 3.55521359670516 & 2.60653059785415 & 9.49439294866952 \\
H & 4.40659615691667 & 1.94737030089579 & 9.42989064897139 \\
\hline
\end{tabular}




\begin{tabular}{|clcc}
\hline 23 & & & \\
BODIPY 14 & & \\
B & 3.35185709079135 & 2.67201170078999 & 6.89787358285596 \\
N & 2.28673646577063 & 3.38806972002752 & 6.00396593798779 \\
N & 2.95737221340765 & 3.02888257103591 & 8.36948801039121 \\
F & 4.62431554885785 & 3.13731639685818 & 6.60601266949094 \\
F & 3.28332737635961 & 1.29546752417741 & 6.72449401676502 \\
C & 2.30677473828016 & 3.47354735647453 & 4.66413933309063 \\
H & 3.13708206227390 & 3.07832637770866 & 4.10355130666844 \\
C & 1.14830772152403 & 4.11381686010968 & 4.20889367708283 \\
H & 0.89593557991316 & 4.32954975670868 & 3.18444453052880 \\
C & 0.39448638017868 & 4.42645786700341 & 5.33625802228955 \\
H & -0.55479659975642 & 4.93759021424837 & 5.34613130388218 \\
C & 1.11396682193601 & 3.97044297445096 & 6.45228310769585 \\
C & 0.83057759288824 & 4.06340629902067 & 7.83310300526858 \\
C & 1.76868498944963 & 3.60316604820764 & 8.78228672172666 \\
C & 1.75946162644477 & 3.63421490554543 & 10.18740441347407 \\
H & 0.98194092908156 & 4.03168292699493 & 10.82013269147588 \\
C & 2.95854263712178 & 3.06871960900767 & 10.61346332560887 \\
H & 3.29397619031805 & 2.93270663921728 & 11.62772233533481 \\
C & 3.66707292318285 & 2.71100969845374 & 9.46136062038203 \\
H & 4.63308332876286 & 2.24467010015830 & 9.36702370315739 \\
N & -0.32199942880038 & 4.62347147485025 & 8.24137961661867 \\
H & -1.02104218320994 & 4.89969662168276 & 7.57765248165986 \\
H & -0.55870400477603 & 4.65995635726803 & 9.21561558656393 \\
\hline
\end{tabular}




\begin{tabular}{|c|c|c|c|}
\hline \multicolumn{4}{|c|}{44} \\
\hline \multicolumn{4}{|c|}{ BODIPY 15} \\
\hline C & 1.59675825599976 & -4.19878353565780 & 4.11713463675454 \\
\hline C & 0.33582448692536 & -3.59311615901065 & 4.18889407025162 \\
\hline $\mathrm{C}$ & 0.50831170632337 & -2.23014748776456 & 3.90542120291310 \\
\hline C & 1.89017727512612 & -2.02817241217100 & 3.65608738770514 \\
\hline $\mathrm{N}$ & 2.51744159231435 & -3.26239412863636 & 3.79256419486742 \\
\hline $\mathrm{C}$ & 2.62499744924922 & -0.86887315333777 & 3.30268813952359 \\
\hline $\mathrm{C}$ & 3.98401323692097 & -0.94900654359151 & 3.03471187496215 \\
\hline $\mathrm{C}$ & 4.97201200592971 & -0.00202869878986 & 2.57254183612977 \\
\hline $\mathrm{C}$ & 6.17295279865935 & -0.67905887661068 & 2.44827062521450 \\
\hline C & 5.92188020150502 & -2.03022848271319 & 2.82425640617051 \\
\hline $\mathrm{N}$ & 4.64124934867476 & -2.18440745056174 & 3.16375325582739 \\
\hline B & 4.01445163048323 & -3.51711386545343 & 3.62287620564512 \\
\hline $\mathrm{F}$ & 4.25510458806435 & -4.50263043869520 & 2.64406094961809 \\
\hline $\mathrm{C}$ & 1.96654976849434 & -5.62418153732036 & 4.33466117362188 \\
\hline $\mathrm{C}$ & -0.62091541607277 & -1.25019069548387 & 3.87050478668786 \\
\hline $\mathrm{C}$ & 1.88373182548542 & 0.43470217434203 & 3.21623871101821 \\
\hline $\mathrm{C}$ & 4.79965611265540 & 1.45184665841917 & 2.25235105410382 \\
\hline C & 7.51697557056598 & -0.19554428146062 & 2.01505019704740 \\
\hline $\mathrm{N}$ & 6.82334913515604 & -3.03151346468905 & 2.87143590900145 \\
\hline $\mathrm{F}$ & 4.61265243325805 & -3.92783574677204 & 4.81979905763743 \\
\hline $\mathrm{H}$ & 1.12460867166590 & -6.18260228104013 & 4.73917020711031 \\
\hline $\mathrm{H}$ & 2.80574141702867 & -5.70673308460975 & 5.02594155036383 \\
\hline $\mathrm{H}$ & 2.27917044332479 & -6.09324522146320 & 3.39956048274297 \\
\hline $\mathrm{H}$ & -1.56395768597825 & -1.76277879225019 & 4.05341023743431 \\
\hline $\mathrm{H}$ & -0.70160928266421 & -0.74381399727452 & 2.90706668861333 \\
\hline $\mathrm{H}$ & -0.52560769604505 & -0.47165970582546 & 4.63090091379329 \\
\hline $\mathrm{H}$ & 1.22060402920383 & 0.55357366066738 & 4.06947548858721 \\
\hline $\mathrm{H}$ & 1.26285163022608 & 0.47321166728619 & 2.31783498315031 \\
\hline$H$ & 2.54852132899281 & 1.28611841534891 & 3.20205341870363 \\
\hline $\mathrm{H}$ & 3.85879220107863 & 1.61210607078079 & 1.72602749292123 \\
\hline $\mathrm{H}$ & 5.58073943921054 & 1.74490917781523 & 1.54878129182689 \\
\hline $\mathrm{C}$ & 4.87842856015935 & 2.37115541002922 & 3.47921098646094 \\
\hline $\mathrm{H}$ & 4.17199890705369 & 2.06885609897001 & 4.25186089310123 \\
\hline $\mathrm{H}$ & 4.65835103215727 & 3.40331564631313 & 3.19849064312934 \\
\hline $\mathrm{H}$ & 5.87537381180709 & 2.33899944038838 & 3.92133917137828 \\
\hline $\mathrm{H}$ & 7.71286474547956 & -2.89680339710907 & 2.42726248697376 \\
\hline $\mathrm{H}$ & 6.48924572143852 & -3.97685602771908 & 2.97355397332696 \\
\hline $\mathrm{H}$ & 7.48700582918650 & 0.83324542923210 & 1.66000268722762 \\
\hline $\mathrm{H}$ & 7.91735547439709 & -0.80500874524367 & 1.19863711882638 \\
\hline $\mathrm{H}$ & 8.24186539023855 & -0.23883046321967 & 2.83421474112119 \\
\hline C & -0.96254609126876 & -4.28166124507682 & 4.46864808597288 \\
\hline $\mathrm{H}$ & -0.80500823453721 & -5.28804099988755 & 4.85462165728243 \\
\hline$H$ & -1.57628820045591 & -4.37163897408100 & 3.56738061362942 \\
\hline $\mathrm{H}$ & -1.55519544741754 & -3.74144995607272 & 5.20923251162128 \\
\hline
\end{tabular}




\begin{tabular}{|c|c|c|c|}
\hline \multirow{2}{*}{\multicolumn{4}{|c|}{$\begin{array}{l}40 \\
\text { BODIPY } 16\end{array}$}} \\
\hline & & & \\
\hline $\mathrm{C}$ & 1.55707378139849 & -4.23615828551834 & 4.11241140070730 \\
\hline $\mathrm{C}$ & 0.28645467446859 & -3.61245338597032 & 4.18267378201513 \\
\hline $\mathrm{C}$ & 0.40753352289280 & -2.25398923701613 & 3.88562040762278 \\
\hline $\mathrm{C}$ & 1.79399066243060 & -2.06120482469058 & 3.62215491023709 \\
\hline $\mathrm{N}$ & 2.44191561134797 & -3.29492879248882 & 3.77741817404723 \\
\hline $\mathrm{C}$ & 2.51074065543539 & -0.91484480760893 & 3.25169006465357 \\
\hline C & 3.90158365584299 & -0.96682900605634 & 3.07950878522395 \\
\hline $\mathrm{C}$ & 4.83566458131220 & 0.04710635853122 & 2.72669195407824 \\
\hline $\mathrm{C}$ & 6.05315960745743 & -0.61993632050131 & 2.58171700735236 \\
\hline C & 5.88689719382487 & -1.99329359707445 & 2.89195097428266 \\
\hline $\mathrm{N}$ & 4.59820663468166 & -2.18043736651770 & 3.18397745240141 \\
\hline B & 3.95989029206022 & -3.56132780544199 & 3.53691505074610 \\
\hline $\mathrm{F}$ & 4.11217134369092 & -4.44256317946563 & 2.47549726003063 \\
\hline $\mathrm{C}$ & 1.90445253413490 & -5.66271008816735 & 4.32598459550095 \\
\hline $\mathrm{C}$ & -0.68736425746987 & -1.23988620616921 & 3.92785141718144 \\
\hline $\mathrm{C}$ & 1.77489725668736 & 0.36307136306160 & 2.97093570538156 \\
\hline $\mathrm{C}$ & 4.63380321046265 & 1.52382936573616 & 2.65227260195618 \\
\hline $\mathrm{N}$ & 7.29019578627884 & -0.03335094474443 & 2.15577332775478 \\
\hline C & 6.90204568002299 & -3.07377080715788 & 2.90129729252500 \\
\hline $\mathrm{F}$ & 4.53663206853715 & -4.06814527871358 & 4.69048188114614 \\
\hline $\mathrm{H}$ & 1.17179640512111 & -6.30306530168810 & 3.83808392738936 \\
\hline $\mathrm{H}$ & 1.85705829976314 & -5.89854420680195 & 5.39009036319466 \\
\hline $\mathrm{H}$ & 2.89641013163285 & -5.88581022935346 & 3.94882265458982 \\
\hline $\mathrm{H}$ & -1.54733723983750 & -1.63554742369628 & 4.45672628527599 \\
\hline $\mathrm{H}$ & -1.03102754867778 & -0.98658323670425 & 2.92128552015141 \\
\hline $\mathrm{H}$ & -0.36158790238225 & -0.32240526206165 & 4.41179472549491 \\
\hline $\mathrm{H}$ & 7.41295868426690 & -3.11571280610651 & 1.93866972044298 \\
\hline $\mathrm{H}$ & 6.45243551598033 & -4.03481218041601 & 3.12218055039857 \\
\hline $\mathrm{H}$ & 7.66888447031039 & -2.84701773093881 & 3.64198798527454 \\
\hline $\mathrm{N}$ & -0.92321039566197 & -4.31391378861026 & 4.50537933841288 \\
\hline $\mathrm{O}$ & -0.81986939474781 & -5.36988053595732 & 5.12009922465451 \\
\hline $\mathrm{O}$ & -1.98936587162184 & -3.82455661364161 & 4.14687575012224 \\
\hline $\mathrm{O}$ & 7.24293916546488 & 1.04779557773538 & 1.57731345913543 \\
\hline $\mathrm{O}$ & 8.32514061321478 & -0.65452111649706 & 2.37187814538522 \\
\hline $\mathrm{H}$ & 0.78205999893581 & 0.16363840298470 & 2.58745455489119 \\
\hline $\mathrm{H}$ & 2.29683246416675 & 0.96090168141287 & 2.23383762348973 \\
\hline $\mathrm{H}$ & 1.66802811772106 & 0.96461875716850 & 3.87766097530025 \\
\hline $\mathrm{H}$ & 3.87909181061171 & 1.85756937096909 & 3.35809144071267 \\
\hline $\mathrm{H}$ & 4.33274428878279 & 1.83801766030280 & 1.64952099493866 \\
\hline $\mathrm{H}$ & 5.56559389145850 & 2.03865182787394 & 2.86682271590047 \\
\hline
\end{tabular}




\begin{tabular}{|llll|}
\hline B1 & & & \\
BODIPY 17 & & \\
C & 1.50540350306627 & -4.15594572420523 & 4.58893280585243 \\
C & 0.23757090108594 & -3.56155196778556 & 4.73283731467628 \\
C & 0.35505438368997 & -2.27939541544736 & 4.23150734509023 \\
C & 1.68514411344555 & -2.11925180160527 & 3.77290809275911 \\
N & 2.37220985722825 & -3.30464895415384 & 4.02614203654727 \\
C & 2.32036370662535 & -1.02749506731505 & 3.17575321413046 \\
C & 3.68059294631286 & -1.08780588404300 & 2.85924495488910 \\
C & 4.52089974951280 & -0.14502785533121 & 2.21877715000358 \\
C & 5.78170271257035 & -0.70201739579903 & 2.12944428655049 \\
C & 5.68573594268567 & -1.98195127662115 & 2.70572925054131 \\
N & 4.44117738515231 & -2.22027284657825 & 3.13878599741518 \\
B & 3.90093894794045 & -3.55783568156581 & 3.76260827714307 \\
F & 4.53931082907492 & -3.80114325247217 & 4.96222482704203 \\
Cl & 1.93592837680603 & -5.73191567625521 & 5.06193672414186 \\
Cl & 6.94386976197348 & -3.11623295612270 & 2.84753645504715 \\
H & 6.67346011715757 & -0.27731307043286 & 1.70338096050694 \\
H & 4.21327016675747 & 0.82118593529837 & 1.85979800917388 \\
C & 1.55904510083716 & 0.19514008841644 & 2.85101868567116 \\
H & -0.40986109765434 & -1.52250407514076 & 4.20150516479800 \\
H & -0.62607020958113 & -4.03860834626014 & 5.16126646669313 \\
C & 2.00389764499452 & 1.44717567176725 & 3.28284323889247 \\
C & 1.32494194119486 & 2.60557034657871 & 2.93554718754446 \\
C & 0.19125523343903 & 2.51335885300791 & 2.13909337135633 \\
C & -0.28095570331509 & 1.28170175489670 & 1.70841235807505 \\
C & 0.40179255254837 & 0.12856564402422 & 2.06991784842827 \\
H & 2.88346426011019 & 1.51292726186462 & 3.90722339616797 \\
H & 1.67573149122761 & 3.56768714311444 & 3.27972973982497 \\
Br & -0.72126378141853 & 4.10369885392068 & 1.61975604887352 \\
H & -1.16373592627221 & 1.22309618551233 & 1.08864162254418 \\
H & 0.05175750946693 & -0.83080817803735 & 1.71456978196930 \\
F & 4.04657758333740 & -4.58101231322970 & 2.84866738765082 \\
\hline
\end{tabular}




\begin{tabular}{|c|c|c|c|}
\hline 38 & & & \\
\hline $\mathrm{BOL}$ & DIPY 18 & & \\
\hline $\mathrm{C}$ & 1.27700079638196 & -4.30208452406353 & 4.48183296742564 \\
\hline C & 0.04501767216682 & -3.64077603023148 & 4.60661337133856 \\
\hline $\mathrm{C}$ & 0.25539359763857 & -2.34517647410335 & 4.16304139734382 \\
\hline $\mathrm{C}$ & 1.60648870225097 & -2.24745934953801 & 3.76256067501753 \\
\hline $\mathrm{N}$ & 2.21312192593364 & -3.47838375650536 & 3.98461174000169 \\
\hline $\mathrm{C}$ & 2.33919683499319 & -1.17411430121643 & 3.24262287578605 \\
\hline $\mathrm{C}$ & 3.70869260590492 & -1.30323774483120 & 3.01346488820053 \\
\hline $\mathrm{C}$ & 4.60660843413305 & -0.39319120264082 & 2.40042526820166 \\
\hline $\mathrm{C}$ & 5.83593778592892 & -1.00036673635115 & 2.35360081241289 \\
\hline $\mathrm{C}$ & 5.70482965257324 & -2.29107166597711 & 2.93494207152660 \\
\hline $\mathrm{N}$ & 4.41565943417694 & -2.46396225414736 & 3.32262189509909 \\
\hline B & 3.74125522686794 & -3.78501190820387 & 3.82715377273842 \\
\hline $\mathrm{F}$ & 4.23468719355582 & -4.13309378958087 & 5.08042475553932 \\
\hline $\mathrm{Cl}$ & 1.60174907516985 & -5.91972309326619 & 4.90496332240722 \\
\hline $\mathrm{C}$ & 6.78668731231597 & -3.23060994335487 & 3.08503026900711 \\
\hline $\mathrm{H}$ & 6.75368439609904 & -0.58518530004679 & 1.97313967259106 \\
\hline $\mathrm{H}$ & 4.34944749995907 & 0.58518911116826 & 2.03586990728254 \\
\hline $\mathrm{C}$ & 1.66535627228624 & 0.09316249944277 & 2.89512610095275 \\
\hline $\mathrm{H}$ & -0.46331881497038 & -1.54416247701394 & 4.13743533092561 \\
\hline $\mathrm{H}$ & -0.86036354298029 & -4.08172844412897 & 4.98498541579148 \\
\hline $\mathrm{C}$ & 2.16242380046934 & 1.31674040518918 & 3.35205721421502 \\
\hline $\mathrm{C}$ & 1.58205617384281 & 2.51388718870583 & 2.96134664613904 \\
\hline $\mathrm{C}$ & 0.49210647403557 & 2.49164029836072 & 2.10192262738053 \\
\hline C & -0.04021510782214 & 1.29029889252671 & 1.65410800902420 \\
\hline $\mathrm{C}$ & 0.54660108642136 & 0.09848086554660 & 2.05694238011933 \\
\hline $\mathrm{H}$ & 3.00860767089982 & 1.32991635506667 & 4.02411563351751 \\
\hline $\mathrm{H}$ & 1.97547455447562 & 3.45430700763724 & 3.31895656333921 \\
\hline $\mathrm{Br}$ & -0.27017302195501 & 4.13849040323634 & 1.51872347507124 \\
\hline $\mathrm{H}$ & -0.89033239396375 & 1.28378389957896 & 0.98731091211538 \\
\hline $\mathrm{H}$ & 0.15547881269971 & -0.83902230683488 & 1.68622435798382 \\
\hline $\mathrm{F}$ & 3.92762596102408 & -4.78558284517367 & 2.89246719671775 \\
\hline$S$ & 8.17955518389182 & -3.05277543576060 & 2.04905951268399 \\
\hline $\mathrm{C}$ & 8.99794452038687 & -4.33649555722576 & 2.82634952619345 \\
\hline $\mathrm{C}$ & 8.24762552779648 & -4.88320835026541 & 3.83160518821955 \\
\hline $\mathrm{C}$ & 6.98766370321551 & -4.26145215042183 & 3.97938632339144 \\
\hline $\mathrm{H}$ & 6.25695448559619 & -4.53915571086941 & 4.71989395865108 \\
\hline $\mathrm{H}$ & 9.97756141134617 & -4.63365829096105 & 2.49029264605203 \\
\hline $\mathrm{H}$ & 8.58706909725406 & -5.70366728374538 & 4.44644131959585 \\
\hline
\end{tabular}




\begin{tabular}{|clcc|}
\hline 33 & & & \\
BODIPY 19 & & \\
C & 1.90877696498989 & -4.06772821920634 & 4.53336014032923 \\
C & 0.60087222591279 & -3.57240859247230 & 4.70868091651383 \\
C & 0.58761552479085 & -2.29053429782378 & 4.19374837412496 \\
C & 1.89189322907257 & -2.02394271767371 & 3.69871436332315 \\
N & 2.67195493165513 & -3.14805764699937 & 3.94121554944116 \\
C & 2.43867527708830 & -0.88491576214340 & 3.11421129888877 \\
C & 3.80988531861220 & -0.84330036307782 & 2.79798059492594 \\
C & 4.58080882711541 & 0.19230536577800 & 2.21090666405777 \\
C & 5.87444722073689 & -0.27400080220491 & 2.09862801748795 \\
C & 5.88039207545213 & -1.59253507254201 & 2.59346953643090 \\
N & 4.63870004243435 & -1.92584563861366 & 3.01750805852655 \\
B & 4.17137479537179 & -3.32335451592629 & 3.54774287224384 \\
F & 4.91350575407945 & -3.68494962037542 & 4.66018754305897 \\
C & 7.02628478564555 & -2.50931694971506 & 2.63012118961714 \\
H & 6.73791355664877 & 0.23360991065746 & 1.70535512332792 \\
H & 4.20542017821682 & 1.15389803434488 & 1.90910619348492 \\
C & 1.57970499947559 & 0.27197073256715 & 2.80365826391626 \\
H & -0.23535281016904 & -1.59702676458905 & 4.18217345804600 \\
H & -0.21563109904500 & -4.10291244265024 & 5.16742318427406 \\
C & 1.90652006756117 & 1.55188163037918 & 3.25985983705780 \\
C & 1.10551814648033 & 2.63730078669766 & 2.93873834060742 \\
C & -0.02256960998098 & 2.46357894544193 & 2.14428214230887 \\
C & -0.35476370110307 & 1.19462894256011 & 1.68526990794092 \\
C & 0.43386337409670 & 0.10292359117248 & 2.01999284470968 \\
H & 2.77901670720365 & 1.68714642945632 & 3.88295443179728 \\
H & 1.36664646140486 & 3.61959154053632 & 3.30958353944953 \\
H & -1.22572769204765 & 1.05380097538718 & 1.05928653979004 \\
H & 0.18514611755444 & -0.88164882994951 & 1.64819672822550 \\
F & 4.27740827951635 & -4.27276737865089 & 2.54258021347681 \\
H & -0.63757533236795 & 3.31394547930028 & 1.88161212774080 \\
H & 2.31793465871720 & -5.02559246287476 & 4.81046924617418 \\
O & 8.12462681049175 & -2.17245593223631 & 2.24602692243569 \\
H & 6.83217391438879 & -3.51762835455413 & 3.02570583626614 \\
\hline & & & \\
\hline
\end{tabular}




\begin{tabular}{|c|c|c|c|}
\hline 47 & & & \\
\hline $\mathrm{BO}$ & DIPY 20 & & \\
\hline $\mathrm{C}$ & 1.70232661102332 & -3.68575746721249 & 4.45999065879912 \\
\hline $\mathrm{C}$ & 0.44529240503310 & -3.06274501040218 & 4.63625267709444 \\
\hline C & 0.59411410192735 & -1.72759173177779 & 4.24803692082844 \\
\hline $\mathrm{C}$ & 1.94820949569223 & -1.57373800362411 & 3.81681771938958 \\
\hline $\mathrm{N}$ & 2.59073596993890 & -2.79375666979511 & 3.98343897178593 \\
\hline $\mathrm{C}$ & 2.59007405381835 & -0.51759331586401 & 3.19204524082191 \\
\hline $\mathrm{C}$ & 3.90219181054191 & -0.61235644047406 & 2.75400478664180 \\
\hline $\mathrm{C}$ & 4.74015316712179 & 0.31724528832190 & 2.06973677121574 \\
\hline C & 5.99980959599698 & -0.28561830111364 & 1.94808341271676 \\
\hline $\mathrm{C}$ & 5.90284865163154 & -1.55994697423289 & 2.54876379689313 \\
\hline B & 4.10153125706435 & -3.04381655979695 & 3.67991084846887 \\
\hline $\mathrm{F}$ & 4.79140602383025 & -3.30041769017265 & 4.86404938250462 \\
\hline $\mathrm{C}$ & 7.20602842495646 & 0.26832654384524 & 1.26171571113893 \\
\hline $\mathrm{C}$ & -0.42390041363218 & -0.69938118358951 & 4.27828946633976 \\
\hline $\mathrm{C}$ & -0.77731394220833 & -3.73593620874709 & 5.16621330493003 \\
\hline $\mathrm{F}$ & 4.22922970967987 & -4.11635619060806 & 2.79758046947168 \\
\hline $\mathrm{C}$ & 2.03503461168231 & -5.10542917920487 & 4.75162927399845 \\
\hline $\mathrm{C}$ & 6.96062415953135 & -2.59523151874532 & 2.68271366980846 \\
\hline $\mathrm{H}$ & 7.88322822708691 & -2.26797245595621 & 2.20906337878074 \\
\hline $\mathrm{H}$ & 6.63842133449161 & -3.53365970191668 & 2.22986014106071 \\
\hline $\mathrm{H}$ & 7.15867479335435 & -2.80518062698451 & 3.73535278245354 \\
\hline $\mathrm{H}$ & 3.08318245698370 & -5.32042381134296 & 4.57118818970475 \\
\hline $\mathrm{H}$ & 1.43549653162416 & -5.76709902569838 & 4.12213028986775 \\
\hline $\mathrm{H}$ & 1.79403363794316 & -5.34821918549399 & 5.78942848478073 \\
\hline $\mathrm{H}$ & -1.42916166011079 & -3.02057961862527 & 5.66851901436421 \\
\hline $\mathrm{H}$ & -0.51865530134870 & -4.51884289525166 & 5.87992598881136 \\
\hline $\mathrm{H}$ & -1.36050121348293 & -4.20883401454709 & 4.36994592989623 \\
\hline $\mathrm{H}$ & 6.91726339647280 & 1.00965063684268 & 0.51731739115407 \\
\hline $\mathrm{H}$ & 7.76882298431849 & -0.51550604293378 & 0.75359352077903 \\
\hline $\mathrm{H}$ & 7.88903187810115 & 0.76052860896403 & 1.96071243337655 \\
\hline $\mathrm{H}$ & 2.04712410214059 & 0.40120565339503 & 3.02842789578437 \\
\hline $\mathrm{C}$ & 4.35120655937031 & 1.60737149054531 & 1.54126289457355 \\
\hline $\mathrm{N}$ & 4.65120004370891 & -1.74960895293371 & 3.01138589614153 \\
\hline $\mathrm{S}$ & 2.82849572642927 & 1.83240454534515 & 0.72879305675075 \\
\hline $\mathrm{C}$ & 3.17353979169890 & 3.47636932233230 & 0.38779800618739 \\
\hline $\mathrm{C}$ & 4.40124782083123 & 3.84077694909918 & 0.86212455917510 \\
\hline $\mathrm{C}$ & 5.07285455038995 & 2.77788766311108 & 1.51430605800792 \\
\hline $\mathrm{C}$ & -1.76151890303597 & -0.80664995906263 & 3.97518475498965 \\
\hline $\mathrm{C}$ & -2.47333093404565 & 0.40996949040858 & 4.12175046937916 \\
\hline $\mathrm{C}$ & -1.68027911743026 & 1.43690582255598 & 4.54730183952067 \\
\hline $\mathrm{S}$ & -0.05978085555591 & 0.92486362777268 & 4.78242571967193 \\
\hline $\mathrm{H}$ & -2.20725422927702 & -1.72553318362984 & 3.62628912467204 \\
\hline $\mathrm{H}$ & -3.52658509272573 & 0.52420550235596 & 3.91010917735460 \\
\hline $\mathrm{H}$ & -1.96262850889318 & 2.45872811365142 & 4.73992253626365 \\
\hline $\mathrm{H}$ & 6.04533933562945 & 2.87225513429708 & 1.97309286134160 \\
\hline $\mathrm{H}$ & 2.45719689046008 & 4.08505195235236 & -0.13831852943321 \\
\hline $\mathrm{H}$ & 4.81187006124157 & 4.83380557454152 & 0.75023305174194 \\
\hline
\end{tabular}




\begin{tabular}{|c|c|c|c|}
\hline 61 & & & \\
\hline & DIPY 21 & & \\
\hline $\mathrm{C}$ & 2.00070404783742 & -3.97179366570947 & 4.64016436298528 \\
\hline $\mathrm{C}$ & 0.77218465624935 & -3.29563309224190 & 4.81155443348784 \\
\hline C & 0.94224893926366 & -1.97346195494903 & 4.42564488581151 \\
\hline $\mathrm{C}$ & 2.28676579297822 & -1.87799827758030 & 3.96312940623028 \\
\hline $\mathrm{N}$ & 2.91696484829169 & -3.10226858199077 & 4.14347537242233 \\
\hline $\mathrm{C}$ & 2.88723629847194 & -0.88250131862684 & 3.22081329161342 \\
\hline $\mathrm{C}$ & 4.14596554122564 & -1.02524945779371 & 2.67076599321894 \\
\hline $\mathrm{C}$ & 4.88944190564003 & -0.08267230739334 & 1.90738310371472 \\
\hline C & 6.14815713225329 & -0.63481697209442 & 1.74943165533996 \\
\hline $\mathrm{C}$ & 6.16360984889858 & -1.90583956933222 & 2.37099557435987 \\
\hline B & 4.42690066091031 & -3.36439315691633 & 3.78398774964350 \\
\hline $\mathrm{F}$ & 5.17945295594225 & -3.43136545923248 & 4.95708596515726 \\
\hline $\mathrm{C}$ & -0.03697496010724 & -0.88951672522720 & 4.46263676390208 \\
\hline $\mathrm{F}$ & 4.49754337828652 & -4.55244097286041 & 3.06255571714059 \\
\hline $\mathrm{C}$ & 2.22487997651659 & -5.38655102372743 & 4.93429651512046 \\
\hline $\mathrm{C}$ & 7.34900386237413 & -2.76564620833711 & 2.38222831597499 \\
\hline $\mathrm{H}$ & 2.34557264608187 & 0.03171277715336 & 3.03748431902664 \\
\hline $\mathrm{C}$ & 4.39053189720608 & 1.19348748412828 & 1.39403578515160 \\
\hline $\mathrm{N}$ & 4.93709305013881 & -2.14491120593125 & 2.91446992926130 \\
\hline C & 3.41444137888965 & 3.65805141020050 & 0.47201139587714 \\
\hline $\mathrm{C}$ & 4.69891738348409 & 3.56432968785001 & 0.99549781343018 \\
\hline $\mathrm{C}$ & 5.18176261328161 & 2.34686968203227 & 1.45179753907165 \\
\hline $\mathrm{C}$ & -1.39324711537304 & -1.14883239550652 & 4.23084657407105 \\
\hline $\mathrm{C}$ & -2.32659211372602 & -0.12326123985416 & 4.25331451489340 \\
\hline $\mathrm{C}$ & -1.92622919492429 & 1.18446489386044 & 4.50822394096896 \\
\hline $\mathrm{H}$ & -1.70769122682316 & -2.15953627895355 & 4.00645876678121 \\
\hline $\mathrm{H}$ & -3.36886331180004 & -0.34258344129605 & 4.06059627203628 \\
\hline $\mathrm{H}$ & 6.17234689175322 & 2.28659046966211 & 1.88243821418798 \\
\hline $\mathrm{H}$ & 5.32316040912595 & 4.44629979936018 & 1.05991615668111 \\
\hline $\mathrm{C}$ & 1.20565167285265 & -6.29674304939460 & 4.62490223682100 \\
\hline $\mathrm{C}$ & 1.36589026956142 & -7.65209278238676 & 4.86457246291699 \\
\hline C & 2.54329674089392 & -8.12269063525938 & 5.43474045332588 \\
\hline $\mathrm{C}$ & 3.55122586907801 & -7.22447486887511 & 5.76558854188308 \\
\hline $\mathrm{C}$ & 3.40010311926220 & -5.86924836064929 & 5.51832747087705 \\
\hline $\mathrm{C}$ & -0.58671559698515 & 1.45516340574300 & 4.75673431596394 \\
\hline $\mathrm{H}$ & -2.65441844955358 & 1.98473588821151 & 4.52064538306076 \\
\hline $\mathrm{C}$ & 0.34731691239201 & 0.42934487172599 & 4.74129937646282 \\
\hline $\mathrm{H}$ & -0.26677249742450 & 2.46534168318241 & 4.97693676082258 \\
\hline $\mathrm{H}$ & 1.37938647442587 & 0.64494207437359 & 4.97976614769611 \\
\hline C & 3.11066624213771 & 1.29703455339197 & 0.83530064489711 \\
\hline $\mathrm{C}$ & 2.62639565722947 & 2.51693651440567 & 0.38693774827507 \\
\hline $\mathrm{H}$ & 3.03390856373330 & 4.60918527031840 & 0.12348771663482 \\
\hline $\mathrm{H}$ & 2.50440126745593 & 0.40823861990042 & 0.72628777420914 \\
\hline $\mathrm{H}$ & 1.63302524510423 & 2.57467436092076 & -0.03791039385980 \\
\hline $\mathrm{C}$ & 8.60218604345189 & -2.13134650998845 & 2.37555554412915 \\
\hline $\mathrm{C}$ & 9.77551537729910 & -2.86426640623160 & 2.32339928110147 \\
\hline $\mathrm{C}$ & 9.72727742949520 & -4.25330220968827 & 2.27242973961758 \\
\hline $\mathrm{C}$ & 8.49504874069335 & -4.89433771633164 & 2.27692527493300 \\
\hline C & 7.31524800860497 & -4.16446659926779 & 2.33194253035125 \\
\hline $\mathrm{H}$ & 0.29761913289917 & -5.93545255069936 & 4.16103403012469 \\
\hline $\mathrm{H}$ & 0.57522865573044 & -8.34093324373093 & 4.59696487174761 \\
\hline
\end{tabular}




\begin{tabular}{|llll|}
\hline$H$ & 2.67502417086929 & -9.18053367359371 & 5.62252978761616 \\
$H$ & 4.46688062187350 & -7.58338410966623 & 6.21656396124471 \\
$H$ & 4.19080969840224 & -5.18269169370648 & 5.77539465959411 \\
H & 6.36900901253299 & -4.67789390730246 & 2.32038786462952 \\
$H$ & 8.65221463339046 & -1.05250723510827 & 2.43376141436974 \\
$H$ & 10.72797403815384 & -2.35038977593115 & 2.32600498699914 \\
$H$ & 10.64220805852888 & -4.82934707697569 & 2.22660579685967 \\
$H$ & 8.44627011084911 & -5.97468912380806 & 2.22824999331100 \\
H & -0.11253374352751 & -3.73358535113791 & 5.24097997329312 \\
H & 6.95967032824249 & -0.21950326113319 & 1.17833332252999 \\
\hline
\end{tabular}


53

BODIPY 22
C 2.01742514978591
$-3.83157376452815$
4.93112078681948
C 0.72242815468536
$-3.24871116297624$
5.10543646796779
C 0.80864487669820
$-1.89883412595415$
C 2.14441611256197
$-1.70893804413252$
4.67047913579403
N 2.83808462491680
$-2.90181339076577$
4.21008112576757
C 2.73021246871693
$-0.64818101559672$
4.40115675436840
C 3.97835340080158
$-0.72881781695272$
3.55041013400406
C 4.71499352794279
0.23831217426892
2.96673847454753
C 5.91528239737420
$-0.39824321459035$
2.22607218830582
C 5.87989418087312
$-1.73351529070176$
1.81446390276729
B 4.36087124338002
$-3.07136143170098$
2.32166834234656
F 5.15682944179993
$-2.89342753564801$
4.01079560311198
F 4.53366242001059
$-4.31780152520613$
5.13646894632212
C 2.39750442559418
$-5.19629920374789$
3.44838422076467
C 6.93562582896784
$-2.72995644148446$
5.29017861262965
H 2.17054641470954
0.27051054061111
2.13987020975219
C 4.45989464997308
1.56772539556167
3.44098613835529
N 4.72283960680563
$-1.90970614672662$
1.87062743202940
C 1.55403510681973
$-6.25669033246205$
2.99607741277496
C 1.88120419848606
$-7.56125790738635$
4.93669824059740
C 3.04848368318000
$-7.82581795545207$
5.27523545886943
C 3.88522309432348
$-6.77708971995090$
5.98273172361502
6.34752979918264
$-5.47276756412035$
6.00549606705985
C 3.56750627083693
C 8.26341505045902
$-2.35436155334768$
2.38157802008980
C 9.29769474244408
$-3.26145085128996$
2.21144215473334
$-4.55430532113566$
1.78101949933587
$-4.92770871081932$
1.50944253322033
C 7.71392282628452
C 6.67617755125920
H 0.65911053840364
$-4.02481645970169$
1.67999476292693
$-6.05765051087058$
4.36395217447674
$-8.37196727865119$
4.97659425724707
$-8.84237953448912$
6.24850332395403
H 3.30815296650392
H 4.79429461792377
H 4.22430021154587
H 5.66257903925964
$-6.97611631134133$
6.89878217117677
$-4.66415195938521$
6.28821874114278
$-4.32120896146603$
1.46357399255279
H 8.47548835834855
$-1.35715859843956$
2.74091587086257
H 10.31451065823559
$-2.95947274434329$
2.42572797692690
H 9.83042055470119
$-5.26554566875132$
1.65245915484400
H 7.49540511401512
$-5.92768365562830$
1.15882110965900
C 6.84232228019532
0.28610975904720
1.01425555504989
C 6.57453838683081
1.59344998228548
0.67033902089704
C 5.39168964009606
2.23140548730426
1.10020345395413
H 7.74411192399974
$-0.20292402848883$
0.67366044867770
H 7.27871139737298
2.14315840966284
0.06016894173944
H 5.21172687079456
3.25870734659672
0.81125040806446
H 3.55548858288932
C -0.31044149369176
2.06707457923672
2.19419472611044
$-1.06157535162794$
4.73943576428294
$-1.57925741868344$
5.23635545706372
$-2.91606979323394$
5.67513595239390
C -1.57459553454500
C -0.48016760286228
$-3.75192293052470$
5.62160937570971
H -2.51209389386552
$-3.28558814065101$
6.06869309879678
H -0.54808846364169
$-4.77070218334465$
5.97571254003230 
\begin{tabular}{llll}
\hline H & -0.25718473299959 & -0.03119407588101 & 4.41235443283756
\end{tabular}

$\begin{array}{llll}\text { H } & -2.36526520222417 & -0.94729804239498 & 5.2982579034879\end{array}$ 


\begin{tabular}{|llll|}
\hline \multicolumn{2}{|l|}{ BODIPY 23} & & \\
C & 0.73814223941048 & -3.21260331847515 & 4.79939760790038 \\
C & 0.81917807301338 & -1.91052020043946 & 4.31593413437432 \\
C & 2.13624559278085 & -1.72318510508005 & 3.85409425074077 \\
N & 2.84050880906487 & -2.89621933800621 & 4.06995289136913 \\
C & 2.73826567364240 & -0.65839290299159 & 3.18400845639405 \\
C & 4.00760197566600 & -0.73922262749626 & 2.67194809942963 \\
C & 4.76327108510551 & 0.18406707716250 & 1.87885096264092 \\
C & 5.97239431098808 & -0.46891839547784 & 1.52778470048492 \\
C & 5.91017505591672 & -1.75441966748141 & 2.14435046229339 \\
B & 4.35428377835773 & -3.10280570768719 & 3.79953694959900 \\
F & 5.08971113291095 & -2.94935096718754 & 4.96360281141571 \\
F & 4.56339304110986 & -4.32827621918061 & 3.20394952819744 \\
H & 2.17043230525710 & 0.24775326839082 & 3.02098902936070 \\
C & 4.52581664254483 & 1.49065294632781 & 1.44538191527263 \\
N & 4.77276292018558 & -1.91002227098533 & 2.80985785207426 \\
C & 6.93447804684743 & 0.16138855742350 & 0.73331390831735 \\
C & 6.68026414148158 & 1.45075740024335 & 0.31109896532931 \\
C & 5.48607994605797 & 2.10754103140922 & 0.66630642943864 \\
H & 7.84987948662933 & -0.34637158290736 & 0.46225905716000 \\
H & 7.40674933115118 & 1.97024175172061 & -0.29899188105406 \\
H & 5.32020880798781 & 3.11962814491109 & 0.32113958094556 \\
H & 3.61837000522540 & 2.01294627124424 & 1.71979412898838 \\
C & 2.00436626587068 & -3.78697211118723 & 4.63163967201081 \\
Cl & 7.12176017233830 & -2.94051160908915 & 2.07031279672923 \\
H & -0.12479653943384 & -3.70228330603316 & 5.21682180268849 \\
H & 2.34620694209763 & -4.77673607330398 & 4.88395344467754 \\
H & 0.03275075779218 & -1.17459504582359 & 4.27118244322146 \\
\hline
\end{tabular}




\begin{tabular}{|c|c|c|c|}
\hline \\
\hline \multicolumn{4}{|c|}{ BODIPY 24} \\
\hline C & 1.26884432097849 & -3.40334708788242 & 4.88009654149870 \\
\hline $\mathrm{C}$ & 1.22466211139288 & -2.21174837933957 & 4.16296899303339 \\
\hline $\mathrm{C}$ & 2.55451629706944 & -1.95967998221524 & 3.74177087908932 \\
\hline $\mathrm{N}$ & 3.36679415538631 & -2.99384690593852 & 4.19561704176469 \\
\hline $\mathrm{C}$ & 3.09025154699702 & -0.91524803131585 & 3.00361329495921 \\
\hline $\mathrm{C}$ & 4.41770037213282 & -0.88854441283839 & 2.63172044785064 \\
\hline $\mathrm{C}$ & 5.14324412343279 & 0.03994167912285 & 1.81898606808681 \\
\hline $\mathrm{C}$ & 6.44269480109693 & -0.49812540891549 & 1.64881275965459 \\
\hline $\mathrm{C}$ & 6.47593020226856 & -1.73247895384047 & 2.37945631537131 \\
\hline B & 4.90083570126174 & -3.10397595024364 & 3.95677783288431 \\
\hline $\mathrm{F}$ & 5.59476533050640 & -2.92502874591952 & 5.14800380931754 \\
\hline $\mathrm{F}$ & 5.20463933113095 & -4.32830968296093 & 3.37814810007206 \\
\hline C & 4.80579897303046 & 1.25610823467525 & 1.21910432810525 \\
\hline $\mathrm{N}$ & 5.27528072428732 & -1.93070538845384 & 2.95968333358263 \\
\hline $\mathrm{C}$ & 7.39532560775078 & 0.15339378651805 & 0.86083239662768 \\
\hline $\mathrm{C}$ & 7.04292831632119 & 1.34807298702213 & 0.26661054948571 \\
\hline $\mathrm{C}$ & 5.75774285746258 & 1.89504026349452 & 0.44880303535865 \\
\hline C & 2.59921152933653 & -3.85939521633162 & 4.88888839799839 \\
\hline $\mathrm{C}$ & 7.54024292332962 & -2.62831302335038 & 2.45445071036958 \\
\hline $\mathrm{C}$ & 3.15114322211269 & -5.07904206503886 & 5.53439213804305 \\
\hline $\mathrm{C}$ & 0.02977590012007 & -1.36572618527398 & 3.87422497266233 \\
\hline C & 8.45919634447453 & -3.41371384298856 & 2.44872196297179 \\
\hline C & 9.45464331354387 & -4.41763982168402 & 2.44048736788746 \\
\hline C & 10.79545337547744 & -4.11316719511018 & 2.16517587362277 \\
\hline C & 11.74717421634056 & -5.12012576594159 & 2.15100493014869 \\
\hline C & 11.37642041982877 & -6.43769091892381 & 2.40087273820649 \\
\hline C & 10.04748465571042 & -6.74801446153009 & 2.67219684210933 \\
\hline C & 9.08879204266361 & -5.74894292314917 & 2.69709809721023 \\
\hline $\mathrm{H}$ & 2.37713436717722 & -5.58797736907496 & 6.10612733298517 \\
\hline $\mathrm{H}$ & 3.97633121795135 & -4.81950678562750 & 6.19993598270931 \\
\hline $\mathrm{H}$ & 3.55390792856915 & -5.76310732986355 & 4.78529574772075 \\
\hline $\mathrm{H}$ & 0.27024931552116 & -0.53774594879236 & 3.20831609226409 \\
\hline $\mathrm{H}$ & -0.38836666406509 & -0.94173564077634 & 4.79071243007728 \\
\hline $\mathrm{H}$ & -0.75860102509682 & -1.95453424958294 & 3.39973628581927 \\
\hline $\mathrm{H}$ & 11.07599931922132 & -3.08770769912428 & 1.96671007341957 \\
\hline $\mathrm{H}$ & 9.75704940789918 & -7.77213427278062 & 2.86651592423921 \\
\hline $\mathrm{H}$ & 8.05302426925716 & -5.97512975973184 & 2.91000282548882 \\
\hline $\mathrm{H}$ & 12.78036049186734 & -4.87800957180239 & 1.93908728243546 \\
\hline $\mathrm{H}$ & 12.12211416162873 & -7.22149275594450 & 2.38377728830008 \\
\hline $\mathrm{H}$ & 8.38014597676155 & -0.27223658654685 & 0.72168354977031 \\
\hline $\mathrm{H}$ & 3.82535131414424 & 1.69321531611270 & 1.35895926299697 \\
\hline $\mathrm{H}$ & 7.76018833634697 & 1.87702919014673 & -0.34662477846062 \\
\hline $\mathrm{H}$ & 5.51524870958971 & 2.83753411367822 & -0.02427669163814 \\
\hline $\mathrm{H}$ & 2.44586402207760 & -0.10995095461674 & 2.67987049458122 \\
\hline $\mathrm{H}$ & 0.44173613570446 & -3.90632629731945 & 5.35503113931868 \\
\hline
\end{tabular}




\begin{tabular}{|llll|}
\hline B3 & & & \\
BODIPY 25 & & \\
C & 1.08250774440266 & -3.75250347933901 & 5.01476906039169 \\
C & 0.95675836871485 & -2.51721944241062 & 4.42486638738856 \\
C & 2.28490905224891 & -2.19821643456706 & 3.99249561816679 \\
N & 3.16132517970847 & -3.22924654046657 & 4.34653500035613 \\
C & 2.74662857956644 & -1.12054707499567 & 3.25161684954146 \\
C & 4.05914536863795 & -1.05082402534121 & 2.80814136324907 \\
C & 4.68249920667052 & -0.09225438076558 & 1.94724870522149 \\
C & 5.95682405935238 & -0.57708163950166 & 1.77071845949754 \\
C & 6.11339657744745 & -1.77216511789975 & 2.49817997961571 \\
B & 4.69410730293320 & -3.25449094412508 & 4.09274672986014 \\
F & 5.38769468244324 & -3.08418393786868 & 5.28896980074942 \\
F & 5.05007786924456 & -4.45468614046602 & 3.48710012580103 \\
N & 4.96759808286015 & -2.06543120610024 & 3.13047783965582 \\
O & 7.11343561247195 & -0.27988788424635 & 1.10844794880242 \\
C & 2.42696798135419 & -4.16723398353959 & 4.96020373214995 \\
C & 7.43819800119458 & -2.22452063280419 & 2.27193142239844 \\
H & 2.05688948819691 & -0.33545790568865 & 2.97363828338924 \\
H & 0.08616696085820 & -1.90174719038980 & 4.27678177117515 \\
C & 2.49137715512089 & -5.45080636990518 & 5.55791138465440 \\
C & 1.20649497915846 & -5.73751318534388 & 5.92666661504423 \\
O & 0.32153065203458 & -4.71771930693728 & 5.61077225361592 \\
C & 0.61434723799481 & -6.92548405452336 & 6.57508272631969 \\
H & 1.39679162305580 & -7.64171079897274 & 6.81797744630796 \\
H & -0.11305056381126 & -7.40769480875837 & 5.91712485400685 \\
H & 0.09263001510674 & -6.64793704917548 & 7.49389333461250 \\
H & 4.20946324069343 & 0.78463016942696 & 1.53983922979472 \\
C & 7.99410554697681 & -1.29871729605044 & 1.43469848402613 \\
H & 7.92694686945851 & -3.10183231339582 & 2.65581094161851 \\
H & 3.34703928337633 & -6.08857296779173 & 5.69310837543496 \\
C & 9.33931390173781 & -1.21684868040419 & 0.83105198454820 \\
H & 9.78853066645300 & -0.23834478089338 & 1.01767407615049 \\
H & 9.29497318660236 & -1.36521218594038 & -0.25221953001230 \\
H & 9.98266608773512 & -1.98393841081907 & 1.25672874646767 \\
\hline
\end{tabular}




\begin{tabular}{|llll|}
\hline BO & & & \\
BODIPY 26 & & \\
C & 0.46941413928578 & -3.78463240394182 & 5.08288450084464 \\
C & 0.61748989759035 & -2.47984946051095 & 4.61750388507503 \\
C & 2.00454922225487 & -2.34207730212054 & 4.15712782216499 \\
N & 2.65859663385269 & -3.52186426776622 & 4.38886577831290 \\
C & 2.58339332060917 & -1.27919393913322 & 3.53986358550180 \\
C & 3.90592014857291 & -1.35571397554112 & 3.01369958178265 \\
C & 4.46215145056609 & -0.31508282045534 & 2.24727651827723 \\
C & 5.71685473979997 & -0.44821529141535 & 1.70188054381887 \\
B & 4.16646211102661 & -3.70553021466439 & 4.18332549708588 \\
F & 4.87271877606710 & -3.56165283745246 & 5.36735858559682 \\
F & 4.47641105678892 & -4.87202323609581 & 3.51919824074180 \\
N & 4.64019576646446 & -2.48176515879161 & 3.22075975508666 \\
C & 1.78754656833402 & -4.47223541936209 & 4.96654532240415 \\
H & 2.01654857502328 & -0.37810682472130 & 3.36483199856947 \\
H & 3.87418704449546 & 0.57711701801352 & 2.08621069651792 \\
C & 5.86213027606219 & -2.61485263918172 & 2.67345041459210 \\
C & 6.43574664404032 & -1.62923951722170 & 1.90952298916528 \\
O & 2.07862886142378 & -5.59323330185261 & 5.29545063396203 \\
C & -0.45111534948636 & -1.59707844346943 & 4.63079417550210 \\
C & -1.67382666114430 & -2.06280273281673 & 5.10704564372109 \\
C & -1.82202998408709 & -3.37710035182057 & 5.55285000380670 \\
C & -0.74200412437838 & -4.25617397267452 & 5.54868350324106 \\
H & -0.34892281587572 & -0.57797791689871 & 4.28179972632727 \\
H & -2.52556778775409 & -1.39563336746081 & 5.12939365937693 \\
H & -2.78550888536596 & -3.71282787167381 & 5.91260793574576 \\
H & -0.83841265404229 & -5.27522933496504 & 5.89771062470614 \\
H & 6.35630864238044 & -3.55299552568821 & 2.87742077336689 \\
H & 7.41775573448165 & -1.77834084958251 & 1.48747615107905 \\
H & 6.14141865301414 & 0.35220195926508 & 1.11034145362677 \\
\hline & & & \\
\hline
\end{tabular}




\begin{tabular}{|c|c|c|c|}
\hline \multicolumn{4}{|c|}{35} \\
\hline \multicolumn{4}{|c|}{ BODIPY 27} \\
\hline C & 0.50722071357042 & -3.84382879314285 & 5.09235140790198 \\
\hline $\mathrm{C}$ & 0.67673561978393 & -2.55484803042668 & 4.59162960867386 \\
\hline $\mathrm{C}$ & 2.06255974765780 & -2.45867013585490 & 4.12091651603027 \\
\hline $\mathrm{N}$ & 2.69668115186401 & -3.63450325255592 & 4.37848675962544 \\
\hline $\mathrm{C}$ & 2.67309873614798 & -1.42874896604163 & 3.48113201541565 \\
\hline $\mathrm{C}$ & 4.01284373510326 & -1.52601543563755 & 3.01246055789239 \\
\hline $\mathrm{C}$ & 4.55035825350792 & -0.42737390325374 & 2.28612607962024 \\
\hline $\mathrm{C}$ & 5.80820242447879 & -0.48040294895055 & 1.78682122652657 \\
\hline B & 4.17621434928352 & -3.91275766343595 & 4.07420146069067 \\
\hline $\mathrm{F}$ & 4.91260557218178 & -4.00352898935910 & 5.24473763339963 \\
\hline $\mathrm{F}$ & 4.29906968055653 & -5.03861843941535 & 3.28052279211394 \\
\hline $\mathrm{N}$ & 4.75452330350809 & -2.63244435029759 & 3.23147237540953 \\
\hline $\mathrm{C}$ & 1.81106033941580 & -4.56074205106682 & 4.97774179854412 \\
\hline $\mathrm{H}$ & 2.13108851903019 & -0.51942244418427 & 3.27398504263881 \\
\hline $\mathrm{H}$ & 3.91848601781252 & 0.43589631440332 & 2.13496391580586 \\
\hline $\mathrm{C}$ & 6.05151783922177 & -2.70965873319077 & 2.74157137889383 \\
\hline C & 6.60365905945014 & -1.63144486753723 & 1.99793880805736 \\
\hline $\mathrm{O}$ & 2.08098553466454 & -5.68301218716665 & 5.31773518300570 \\
\hline C & -0.37049316543613 & -1.64766210821133 & 4.59323706215645 \\
\hline C & -1.59827842022602 & -2.07395451625434 & 5.09330889453879 \\
\hline C & -1.76930926155409 & -3.37273068359988 & 5.57458940168314 \\
\hline C & -0.70859831296383 & -4.27558694295384 & 5.58362486599738 \\
\hline $\mathrm{H}$ & -0.24810740751940 & -0.64030853551038 & 4.21759498747532 \\
\hline $\mathrm{H}$ & -2.43537151921397 & -1.38824190165496 & 5.10650341985704 \\
\hline $\mathrm{H}$ & -2.73586103686256 & -3.67835198496029 & 5.95235685391078 \\
\hline $\mathrm{H}$ & -0.82418123640764 & -5.28245486953324 & 5.96083630865386 \\
\hline C & 7.91380008466405 & -1.72425061289357 & 1.48844188678482 \\
\hline $\mathrm{H}$ & 6.21898067545626 & 0.34859221033728 & 1.22407910563443 \\
\hline C & 8.67035454940960 & -2.84599529063247 & 1.70699828823284 \\
\hline C & 8.12930798017127 & -3.90439722419745 & 2.45416922660410 \\
\hline C & 6.85493805299863 & -3.84533317083720 & 2.96659897023764 \\
\hline $\mathrm{H}$ & 8.72502119372451 & -4.78898107633653 & 2.63625534880372 \\
\hline $\mathrm{H}$ & 6.46770520260883 & -4.67463305302555 & 3.53083590329020 \\
\hline $\mathrm{H}$ & 8.30632923257938 & -0.88942734161897 & 0.92122855342711 \\
\hline $\mathrm{H}$ & 9.67232279133214 & -2.91928802100307 & 1.30714636246651 \\
\hline
\end{tabular}




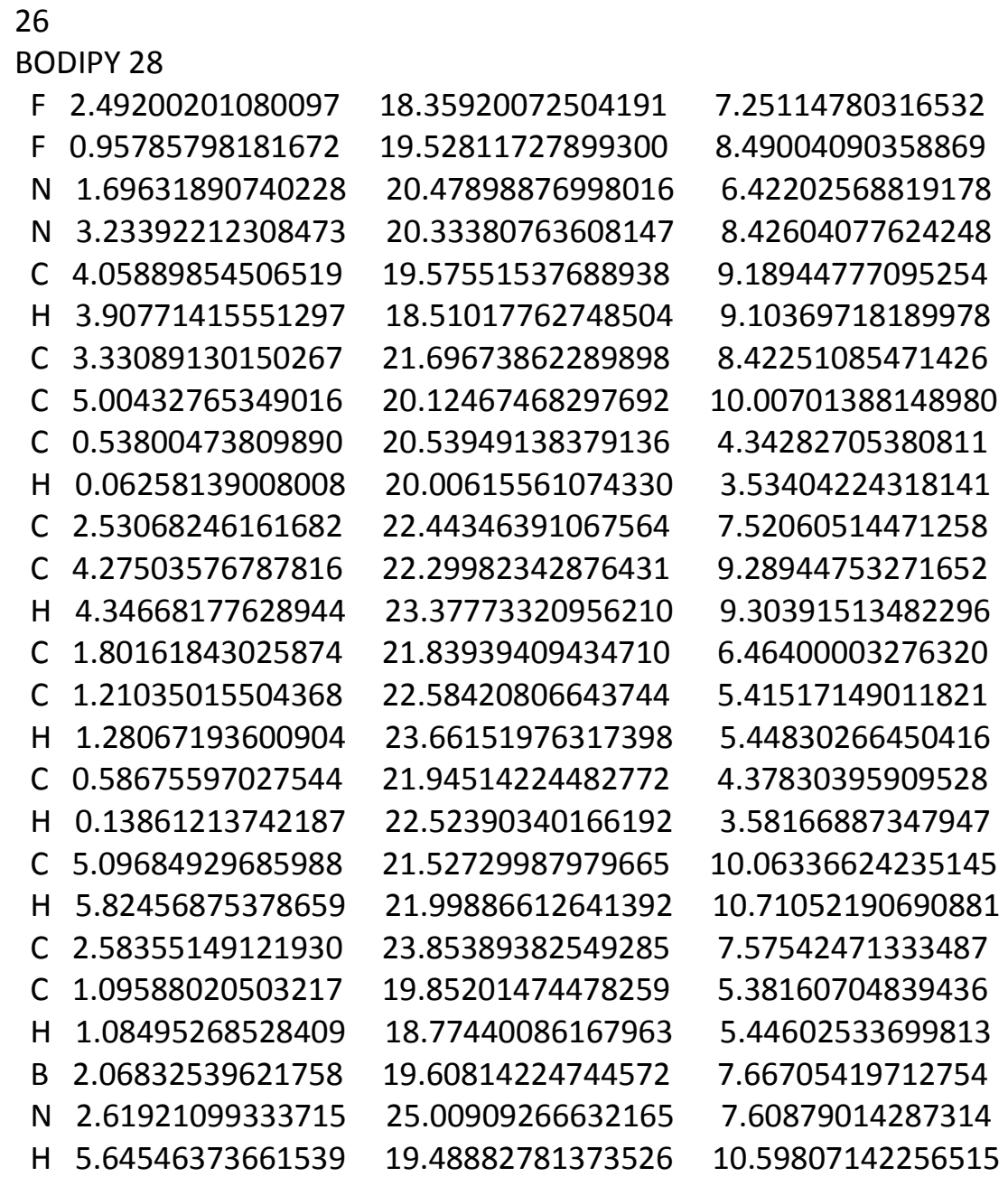




\begin{tabular}{|c|c|c|c|}
\hline 63 & & & \\
\hline & DIPY 29 & & \\
\hline $\mathrm{C}$ & 1.86303277920036 & -3.89491247125749 & 4.74925185745860 \\
\hline $\mathrm{C}$ & 0.60948190523164 & -3.24446901591155 & 4.85062730009639 \\
\hline C & 0.76277271726092 & -1.95023286400994 & 4.37008102561631 \\
\hline $\mathrm{C}$ & 2.13234401145477 & -1.82471198183845 & 3.99201485200130 \\
\hline $\mathrm{N}$ & 2.78524727556073 & -3.02866887737217 & 4.24532512067930 \\
\hline $\mathrm{C}$ & 2.78385986979228 & -0.76010778199258 & 3.39561890010095 \\
\hline $\mathrm{C}$ & 4.13736136074995 & -0.79085453189891 & 3.11850360822722 \\
\hline $\mathrm{C}$ & 4.95555996092650 & 0.11227232535387 & 2.36976083165817 \\
\hline C & 6.24276541889074 & -0.41574791816047 & 2.40481054005165 \\
\hline $\mathrm{C}$ & 6.20149578029135 & -1.60062134788088 & 3.17996620392460 \\
\hline B & 4.35599207902806 & -3.09188024054981 & 4.32678537530612 \\
\hline $\mathrm{F}$ & 4.70494054432611 & -3.06952633811748 & 5.67866721466832 \\
\hline $\mathrm{C}$ & 7.50869877244613 & 0.01735150069139 & 1.74448206270415 \\
\hline $\mathrm{C}$ & -0.26982688717091 & -0.94023277199711 & 4.27567513671109 \\
\hline $\mathrm{C}$ & -0.56558624598179 & -3.92277898980903 & 5.46943454954537 \\
\hline $\mathrm{F}$ & 4.87437599215926 & -4.21715651364649 & 3.69679737572163 \\
\hline C & 1.98886734311427 & -5.29920304343979 & 5.11926885524346 \\
\hline $\mathrm{C}$ & 7.41231439477766 & -2.35797571455783 & 3.45605524101338 \\
\hline $\mathrm{H}$ & 2.20907548846663 & 0.11176108897379 & 3.12264583870061 \\
\hline C & 4.53269985909565 & 1.31003231827379 & 1.67414100055494 \\
\hline $\mathrm{N}$ & 4.92845553975927 & -1.83132830363824 & 3.58712875322156 \\
\hline $\mathrm{S}$ & 2.99297267450279 & 1.42893665679887 & 0.87100474538706 \\
\hline $\mathrm{C}$ & 3.32322331910178 & 3.01736115087655 & 0.31560609266740 \\
\hline $\mathrm{C}$ & 4.55979799080707 & 3.43956371750386 & 0.71175801847633 \\
\hline C & 5.24792903028378 & 2.47009245720693 & 1.48103645028231 \\
\hline C & -1.58317755522098 & -1.11172438703555 & 3.90691792723487 \\
\hline C & -2.33658986970736 & 0.08785617636323 & 3.93485940089602 \\
\hline C & -1.59836132228259 & 1.16459334324345 & 4.33576444660426 \\
\hline $\mathrm{S}$ & 0.02039097525647 & 0.72168573639647 & 4.69265681163753 \\
\hline $\mathrm{H}$ & -1.97772809811033 & -2.06660955871149 & 3.59344207117149 \\
\hline $\mathrm{H}$ & -3.37860279879475 & 0.15333966103594 & 3.65761312037453 \\
\hline $\mathrm{H}$ & -1.92047691722583 & 2.18712504114823 & 4.44385828110953 \\
\hline $\mathrm{H}$ & 6.22778297806094 & 2.62638539521168 & 1.90569778586559 \\
\hline $\mathrm{H}$ & 2.59355503010554 & 3.54869999677949 & -0.27275523875745 \\
\hline $\mathrm{H}$ & 4.96509041786366 & 4.40922276627153 & 0.46204648848183 \\
\hline C & 8.33372830883394 & -1.22500304343881 & 1.39127178964695 \\
\hline $\mathrm{H}$ & 8.08994767436865 & 0.66239616706231 & 2.41477189316241 \\
\hline $\mathrm{H}$ & 7.29414602081516 & 0.60331337797451 & 0.84966691899477 \\
\hline C & 8.49808509674541 & -2.14434604526911 & 2.57574319603534 \\
\hline $\mathrm{H}$ & 9.31159048763924 & -0.93807274643924 & 1.00215227876713 \\
\hline $\mathrm{H}$ & 7.81693839399437 & -1.76715856981560 & 0.59067352394614 \\
\hline C & 9.69613278681359 & -2.80667742756251 & 2.80785201156825 \\
\hline C & 9.84454062258023 & -3.65699961336495 & 3.89771451797743 \\
\hline C & 8.78394241034221 & -3.84960017436729 & 4.77362500781312 \\
\hline C & 7.57510653042711 & -3.20862498474293 & 4.55599960267605 \\
\hline $\mathrm{H}$ & 6.76311385630278 & -3.35101281537751 & 5.24996867026108 \\
\hline $\mathrm{H}$ & 8.89847038316649 & -4.49670552456191 & 5.63284625657469 \\
\hline C & 0.99789705610824 & -5.80447783086482 & 5.99548888110028 \\
\hline C & -0.04845247225055 & -4.87516339913023 & 6.55235435784898 \\
\hline $\mathrm{H}$ & -1.12898505657356 & -4.49223909616591 & 4.72042050080911 \\
\hline $\mathrm{H}$ & -1.24914082703684 & -3.18475566576224 & 5.89200480125379 \\
\hline
\end{tabular}




\begin{tabular}{|llll|}
\hline H & -0.86432984776540 & -5.45053594133348 & 6.99072460141927 \\
H & 0.39718667261761 & -4.27563267454827 & 7.35472561413194 \\
C & 2.94118232388842 & -6.17719947624430 & 4.59060697782078 \\
C & 2.93313066107416 & -7.51991091704615 & 4.93771881889749 \\
C & 1.97280350184560 & -8.00993723400349 & 5.81364666016636 \\
C & 1.01008487558223 & -7.15097514432544 & 6.33157265496233 \\
H & 3.67734738844752 & -5.80788152225283 & 3.89838654270604 \\
H & 3.67502732810851 & -8.18348613836967 & 4.51364248690716 \\
H & 1.96488159346627 & -9.05703566918924 & 6.08791880363138 \\
H & 0.25027426772978 & -7.53280795226655 & 7.00276447157781 \\
H & 10.52621923131067 & -2.64600916512452 & 2.13019896065038 \\
H & 10.79007691739842 & -4.15363745377362 & 4.07264115405708 \\
\hline
\end{tabular}




\begin{tabular}{|c|c|c|c|}
\hline 61 & & & \\
\hline & DIPY 30 & & \\
\hline $\mathrm{C}$ & 1.30534794341728 & -1.50310506262165 & 3.31896908449026 \\
\hline $\mathrm{N}$ & 2.01188938649081 & -2.55962808968032 & 3.87896387958868 \\
\hline C & 1.98624281781945 & -0.36955493004755 & 2.87160718578807 \\
\hline $\mathrm{C}$ & 3.37978436249610 & -0.31438015807902 & 2.92978586384426 \\
\hline B & 3.52421862385512 & -2.69835201358163 & 3.94238999066254 \\
\hline $\mathrm{N}$ & 4.09988295450160 & -1.42378159305374 & 3.35167648000413 \\
\hline $\mathrm{C}$ & 5.39436472743619 & -1.35215415633902 & 2.99776455558692 \\
\hline $\mathrm{C}$ & 5.61786985307803 & -0.04509258243683 & 2.44765201360497 \\
\hline C & 4.34461621465631 & 0.60633632476014 & 2.40055976945151 \\
\hline $\mathrm{C}$ & -0.07893337007354 & -1.75739837584181 & 3.60137781221683 \\
\hline $\mathrm{C}$ & -0.13168146444969 & -2.93173489924528 & 4.41761305328506 \\
\hline $\mathrm{C}$ & 1.22056566722011 & -3.37011548706245 & 4.60098186082616 \\
\hline $\mathrm{C}$ & 1.22064415417196 & 0.78016456337533 & 2.32101365375060 \\
\hline $\mathrm{C}$ & 0.80471432391154 & 1.81995515143155 & 3.15066036768833 \\
\hline $\mathrm{C}$ & 0.07859723416334 & 2.88578622797398 & 2.63257110090897 \\
\hline $\mathrm{C}$ & -0.23291189737487 & 2.92392652218413 & 1.27804254408356 \\
\hline $\mathrm{C}$ & 0.18303193506149 & 1.89205046613645 & 0.44480012837811 \\
\hline $\mathrm{C}$ & 0.90255753649343 & 0.82240270838319 & 0.96420935015880 \\
\hline $\mathrm{H}$ & 1.05186974027478 & 1.78904347212414 & 4.20357624961105 \\
\hline $\mathrm{H}$ & -0.24689082257983 & 3.68352021026532 & 3.28731873123323 \\
\hline $\mathrm{H}$ & -0.79641178819598 & 3.75393819722641 & 0.87245818284563 \\
\hline $\mathrm{H}$ & -0.05986893795340 & 1.91761832926406 & -0.60962180749543 \\
\hline $\mathrm{H}$ & 1.22724764450193 & 0.01662371071960 & 0.31895140290001 \\
\hline $\mathrm{C}$ & -1.26221837098293 & -1.07939331283782 & 3.28038203164480 \\
\hline $\mathrm{C}$ & -2.45732058962176 & -1.56704111018856 & 3.76969775929188 \\
\hline $\mathrm{C}$ & -2.50586208782171 & -2.70809548253223 & 4.59150113916962 \\
\hline $\mathrm{C}$ & -1.35305673307367 & -3.38824994108034 & 4.92582184464307 \\
\hline $\mathrm{C}$ & 6.76076268652251 & 0.60660967512451 & 1.96884736482128 \\
\hline C & 6.63563407237331 & 1.88886432426707 & 1.47526646953769 \\
\hline $\mathrm{C}$ & 5.38479470515879 & 2.52904887757084 & 1.43262386509256 \\
\hline $\mathrm{C}$ & 4.23883775907903 & 1.90087982625964 & 1.87756226140484 \\
\hline C & 6.14914214610135 & -2.58000045276860 & 2.99144824131619 \\
\hline $\mathrm{C}$ & 1.85680861663515 & -4.26157022090520 & 5.53677680172628 \\
\hline $\mathrm{H}$ & -1.24361120324085 & -0.19424207533725 & 2.66267327317508 \\
\hline $\mathrm{H}$ & -3.37855807422137 & -1.05767755570673 & 3.51808866687632 \\
\hline $\mathrm{H}$ & -3.45911143706419 & -3.05382885116272 & 4.96861813955300 \\
\hline $\mathrm{H}$ & -1.39910195762070 & -4.25203500977308 & 5.57253507910116 \\
\hline $\mathrm{H}$ & 7.72753907974001 & 0.12653574039289 & 1.98809427077915 \\
\hline $\mathrm{H}$ & 7.51147703620463 & 2.40939246150777 & 1.11137620531743 \\
\hline $\mathrm{H}$ & 5.32015554496449 & 3.53429925446823 & 1.03777913578854 \\
\hline $\mathrm{H}$ & 3.28459721452118 & 2.40326904546454 & 1.83106890522712 \\
\hline $\mathrm{C}$ & 7.54154578498338 & -2.64368093346234 & 2.83205063351294 \\
\hline C & 8.18611388277146 & -3.85436378275084 & 2.66742141167954 \\
\hline $\mathrm{C}$ & 7.44156991488800 & -5.03558997553323 & 2.63594876848325 \\
\hline C & 6.06678577080879 & -5.00691920555495 & 2.79110032700379 \\
\hline $\mathrm{C}$ & 5.40531476676759 & -3.79597087468542 & 2.99527574774788 \\
\hline $\mathrm{C}$ & 1.21226200503545 & -5.33990158409494 & 6.15821090989930 \\
\hline $\mathrm{C}$ & 1.83398202229205 & -6.05979183216978 & 7.16031760031401 \\
\hline C & 3.11725883213456 & -5.69849507389392 & 7.57448115476757 \\
\hline $\mathrm{C}$ & 3.78353429601419 & -4.64434207877980 & 6.97176822636362 \\
\hline C & 3.18311583476594 & -3.93112061620485 & 5.93567439977566 \\
\hline
\end{tabular}




\begin{tabular}{|llll|}
\hline H & 8.11633582285998 & -1.72963822677136 & 2.85002568777988 \\
$H$ & 9.26118051405484 & -3.88563091303524 & 2.55287457603708 \\
$H$ & 7.94239318197342 & -5.98485640777123 & 2.49536180666337 \\
$H$ & 5.47617348747132 & -5.91271856768002 & 2.77851423321044 \\
O & 4.07240925990507 & -3.80967226709601 & 3.15941977244142 \\
$H$ & 0.22460190579413 & -5.62326631627841 & 5.82448534467586 \\
$H$ & 1.32764285549674 & -6.89668403230052 & 7.62111760825109 \\
$H$ & 3.60696083946928 & -6.25796097287328 & 8.36086444244548 \\
$H$ & 4.78327476938516 & -4.36282020166235 & 7.27192167901115 \\
O & 3.86186900655323 & -2.92668986601942 & 5.35231276205841 \\
\hline
\end{tabular}




\begin{tabular}{|llll|}
\hline BO & & & \\
BODIPY 31 & & \\
C & 1.05676157344097 & -1.65750408411180 & 3.40258074194017 \\
O & 1.88558654883533 & -2.57975998456195 & 3.84106157176067 \\
C & 1.50756911385560 & -0.56233607646050 & 2.71256480760818 \\
C & 2.89548342660168 & -0.33078712920070 & 2.54599728817981 \\
B & 3.27442243341245 & -2.71184421417798 & 3.39278501890087 \\
N & 3.78786862360639 & -1.25417719633043 & 2.97201601523300 \\
F & 3.36509101619027 & -3.47878867186477 & 2.24374296983354 \\
F & 4.05195125953184 & -3.17939204960635 & 4.42548407132480 \\
C & 5.10572185192347 & -1.00264155565932 & 2.91147672975257 \\
C & 5.58465840608070 & 0.16984843463091 & 2.37326018841344 \\
N & 4.71793572339863 & 1.09561472661763 & 1.90050219703084 \\
C & 3.43326194034082 & 0.85587484200719 & 1.98982367341011 \\
C & 7.04986746529029 & 0.44236972120102 & 2.25535165765534 \\
H & 7.63080744829476 & -0.23278579949106 & 2.88121576754318 \\
H & 7.26979183337286 & 1.47055163138446 & 2.54410384759793 \\
H & 7.37786233066948 & 0.30951386580086 & 1.22104864305730 \\
C & -0.34723883418614 & -1.87408933393407 & 3.78691609688004 \\
C & -1.40535162456587 & -1.18861657415751 & 3.17976576416147 \\
C & -2.71320200408801 & -1.43345746149851 & 3.57050284916461 \\
C & -2.98325120847801 & -2.35533992829357 & 4.57815552782102 \\
C & -1.93659518875941 & -3.03166203592851 & 5.19384651063627 \\
C & -0.62946868995322 & -2.80217181117724 & 4.79475171995739 \\
H & 0.18841774929943 & -3.33052135222674 & 5.25993981430951 \\
H & -1.21317518723720 & -0.48349622677697 & 2.38240681827063 \\
H & -3.52492287277183 & -0.91002338473423 & 3.08346922284495 \\
H & -4.00375430762151 & -2.54660823243796 & 4.88397247508381 \\
H & -2.13853639833462 & -3.74268671600276 & 5.98403790061568 \\
H & 2.75228972404987 & 1.61302728323193 & 1.61677156385963 \\
H & 5.75101497772291 & -1.77995811164059 & 3.29325902862254 \\
H & 0.82207287007809 & 0.19980742539951 & 2.38163951853068 \\
\hline
\end{tabular}




\begin{tabular}{|c|c|c|c|}
\hline \\
\hline \multicolumn{4}{|c|}{ BODIPY 32} \\
\hline $\mathrm{C}$ & 1.14843156713015 & -1.49822889819573 & 3.41438142888198 \\
\hline 0 & 1.95938372201244 & -2.43905836752058 & 3.86110920463403 \\
\hline $\mathrm{C}$ & 1.64272553109330 & -0.42905406608381 & 2.70160901943798 \\
\hline $\mathrm{C}$ & 3.03482884949157 & -0.26385904252528 & 2.52262263149737 \\
\hline B & 3.27785533101737 & -2.67696212044195 & 3.27240874627929 \\
\hline $\mathrm{N}$ & 3.88580833518561 & -1.24655793751268 & 2.90468174436245 \\
\hline $\mathrm{F}$ & 3.16586008029754 & -3.37559930286517 & 2.07992022664080 \\
\hline $\mathrm{F}$ & 4.10843099478004 & -3.28884599192785 & 4.18119736676803 \\
\hline C & 5.21503136104595 & -1.05349405353408 & 2.86003963806491 \\
\hline C & 5.74975614295399 & 0.11532890746719 & 2.37382839956456 \\
\hline $\mathrm{N}$ & 4.92707746762842 & 1.09359364840515 & 1.92258703619189 \\
\hline $\mathrm{C}$ & 3.63341337502254 & 0.91203734415825 & 1.99993946441418 \\
\hline $\mathrm{C}$ & 7.22574913464804 & 0.34016537844083 & 2.29342414651518 \\
\hline $\mathrm{H}$ & 7.77436760773608 & -0.43304953946561 & 2.82910818013419 \\
\hline $\mathrm{H}$ & 7.48617522053878 & 1.31129164714948 & 2.71711811839344 \\
\hline $\mathrm{H}$ & 7.55764627161631 & 0.33746665688032 & 1.25284117488721 \\
\hline $\mathrm{C}$ & -0.24820331280448 & -1.68270423639209 & 3.79028344898641 \\
\hline C & -1.30223748745658 & -0.95412302095227 & 3.22562748392169 \\
\hline C & -2.61730826200204 & -1.21559406633223 & 3.55599173549271 \\
\hline $\mathrm{C}$ & -2.94386502250927 & -2.22924829894501 & 4.48275265767982 \\
\hline C & -1.87736715969742 & -2.93167157683749 & 5.08116456611319 \\
\hline $\mathrm{C}$ & -0.57259898514019 & -2.66767599566724 & 4.73336873639837 \\
\hline $\mathrm{H}$ & 0.22712170768883 & -3.22802968278128 & 5.19274311712097 \\
\hline $\mathrm{H}$ & -1.10108468955986 & -0.19597626522439 & 2.48009116374665 \\
\hline $\mathrm{H}$ & -3.39731717605006 & -0.65743998348764 & 3.06241924195542 \\
\hline $\mathrm{N}$ & -4.24361565450061 & -2.54775096355827 & 4.79053632804258 \\
\hline $\mathrm{H}$ & -2.07718492130402 & -3.68787779475032 & 5.82502527647099 \\
\hline $\mathrm{H}$ & 2.99091533411093 & 1.71134814995218 & 1.64679630489260 \\
\hline $\mathrm{H}$ & 5.81963509530870 & -1.87484713642172 & 3.21515316296072 \\
\hline $\mathrm{C}$ & -5.33686878930299 & -1.80655621950530 & 4.19927684727522 \\
\hline $\mathrm{C}$ & -4.53699399008888 & -3.86401181507917 & 5.33548934832462 \\
\hline $\mathrm{H}$ & -5.45819472256439 & -2.01495187251024 & 3.12710789872282 \\
\hline $\mathrm{H}$ & -5.18807832218699 & -0.73373137128425 & 4.32921356605680 \\
\hline $\mathrm{H}$ & -6.26070558466640 & -2.07261276934929 & 4.70758956989324 \\
\hline $\mathrm{H}$ & -4.34753642964891 & -3.91890283245208 & 6.41226775942587 \\
\hline $\mathrm{H}$ & -3.93586042593978 & -4.63344044842610 & 4.84555720535308 \\
\hline $\mathrm{H}$ & -5.58557808876657 & -4.09462297789591 & 5.16067942607173 \\
\hline $\mathrm{H}$ & 0.98949589488285 & 0.36083691547165 & 2.36969862842697 \\
\hline
\end{tabular}




\begin{tabular}{|c|c|c|c|}
\hline \\
\hline \multicolumn{4}{|c|}{ BODIPY 33} \\
\hline & 254467409749 & -1.77330820980211 & 3.6891010661149 \\
\hline & 2.47563197738855 & -2.11571018805438 & 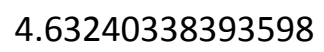 \\
\hline & 35483612 & -1.24693 & 7687 \\
\hline & 0908009 & -1.03 & \\
\hline & 06602858873 & -2.4403 & 166 \\
\hline & 4.36770821286710 & -1.51261588976559 & 282 \\
\hline & 4.05862295354361 & -3.98264433475300 & 3.93285108759737 \\
\hline & 951390489 & & 259 \\
\hline & 5.67 & & \\
\hline & 6.11 & -0.6 & \\
\hline & 5207 & & \\
\hline C & 716335400 & 3492 & \\
\hline & 7.56661503069870 & & \\
\hline & 3263 & & \\
\hline & 7.82 & & \\
\hline$H$ & 7.81 & & \\
\hline C & 97795480 & & \\
\hline$c$ & 10879 & & \\
\hline$C$ & 1151 & & 3030 \\
\hline & -2.4 & 708 & \\
\hline C & 091 & 36 & \\
\hline C & 3139 & 495 & \\
\hline 1 & 0240 & & \\
\hline 7 & 15246 & 74 & \\
\hline t & 4202 & & \\
\hline & -1.6 & & \\
\hline $\mathrm{H}$ & 3.22 & & 338 \\
\hline 1 & 0738 & & \\
\hline$C$ & 4.26 & & \\
\hline C & 18146238 & & \\
\hline c & 5226 & & \\
\hline C & 4.00 & & \\
\hline C & 3.92 & & \\
\hline & 3.76 & & \\
\hline $\mathrm{H}$ & 4.29661370453544 & & 741 \\
\hline $\mathrm{H}$ & 3.90 & & \\
\hline $\mathrm{H}$ & 4.50 & & 546 \\
\hline$c$ & 4.38 & -0 . & 278 \\
\hline C & 5.127 & -0.3 & 756 \\
\hline C & 6.29 & & 1922 \\
\hline C & 6.695 & & 6961 \\
\hline $\mathrm{H}$ & 3.47 & & \\
\hline $\mathrm{H}$ & 4.79 & & \\
\hline & 6.88 & -0.6 & 8.6 \\
\hline & 5.94043593296114 & 79767767 & 6.06 \\
\hline & 7.59 & -2 & 5085 \\
\hline & 6.26 & & \\
\hline & 7 & & \\
\hline & 5.35141183787918 & -1.72 & 3.6249973147 \\
\hline & 36475131281725 & -0.95292911141934 & .737076540893 \\
\hline
\end{tabular}




\begin{tabular}{|c|c|c|c|}
\hline 58 & & & \\
\hline & DIPY 34 & & \\
\hline $\mathrm{C}$ & 1.89295840435908 & -1.53547929756676 & 3.80700443395445 \\
\hline 0 & 2.74559415965811 & -1.77139076898234 & 4.78699475569605 \\
\hline C & 2.34464095301103 & -1.06813202569960 & 2.59618076652937 \\
\hline $\mathrm{C}$ & 3.72016795228816 & -0.80872469363414 & 2.39316697883773 \\
\hline B & 4.12955046863963 & -2.24503228000347 & 4.46371763680763 \\
\hline $\mathrm{N}$ & 4.63540148267094 & -1.28311466145395 & 3.27326788604922 \\
\hline $\mathrm{C}$ & 4.07766852299834 & -3.75782253234056 & 3.90460939740726 \\
\hline $\mathrm{C}$ & 5.09184881122025 & -2.03465924925133 & 5.73661914568685 \\
\hline C & 5.93644290208223 & -1.01098527921917 & 3.09159196049497 \\
\hline $\mathrm{C}$ & 6.37741455914613 & -0.26203953149238 & 2.02393622865136 \\
\hline $\mathrm{N}$ & 5.48741319106392 & 0.22482171230781 & 1.12962094052011 \\
\hline $\mathrm{C}$ & 4.21706956800516 & -0.04050655441864 & 1.31124994727853 \\
\hline $\mathrm{C}$ & 7.82792379422944 & 0.04804045453423 & 1.83376809115083 \\
\hline $\mathrm{H}$ & 8.44438759496989 & -0.52353622415142 & 2.52607646468950 \\
\hline $\mathrm{H}$ & 8.01984261978053 & 1.11023301579095 & 2.00541718321112 \\
\hline $\mathrm{H}$ & 8.13820553776944 & -0.18473452194909 & 0.81400369100780 \\
\hline C & 0.48863657986700 & -1.77432574085778 & 4.12132266195098 \\
\hline $\mathrm{C}$ & -0.50554510075432 & -1.80815726569381 & 3.13626549380511 \\
\hline $\mathrm{C}$ & -1.81169005298992 & -2.13012515376349 & 3.43753147596452 \\
\hline $\mathrm{C}$ & -2.20222831743910 & -2.42901841766599 & 4.76108605079096 \\
\hline C & -1.20631519243315 & -2.35809637762917 & 5.75829660296708 \\
\hline $\mathrm{C}$ & 0.10014803459100 & -2.05519282249486 & 5.43665119889079 \\
\hline $\mathrm{H}$ & 0.85051163800536 & -2.03386737403129 & 6.21375805362013 \\
\hline $\mathrm{H}$ & -0.25189837898339 & -1.60928858979768 & 2.10414623913662 \\
\hline $\mathrm{H}$ & -2.53401113802949 & -2.16207587486893 & 2.63668379366148 \\
\hline $\mathrm{H}$ & -1.45271895118262 & -2.55904770877048 & 6.78937353683222 \\
\hline $\mathrm{H}$ & 3.51126142005429 & 0.36439388882581 & 0.59389829983627 \\
\hline $\mathrm{N}$ & -3.49289009504572 & -2.79143374192212 & 5.05965994262677 \\
\hline C & 4.99388239839065 & -4.27677567333989 & 2.98493612746530 \\
\hline $\mathrm{C}$ & 4.96629639055845 & -5.61345586153514 & 2.59907726256078 \\
\hline $\mathrm{C}$ & 4.00891833413745 & -6.46992919698390 & 3.12930698991535 \\
\hline C & 3.08236424386914 & -5.97728290275970 & 4.04206776851956 \\
\hline $\mathrm{C}$ & 3.11920455055914 & -4.63940004503449 & 4.41717416452883 \\
\hline $\mathrm{H}$ & 2.39037929039879 & -4.27027590913053 & 5.12844810796290 \\
\hline $\mathrm{H}$ & 3.98159763409573 & -7.50997633885096 & 2.82938944747558 \\
\hline $\mathrm{H}$ & 2.33093021229873 & -6.63558880341266 & 4.46084150475139 \\
\hline $\mathrm{H}$ & 5.75221203430920 & -3.63253647855960 & 2.55361752467614 \\
\hline $\mathrm{H}$ & 5.69024717458471 & -5.98626494210834 & 1.88469228671269 \\
\hline $\mathrm{C}$ & 5.34229351919248 & -0.75052458009697 & 6.23953532293156 \\
\hline C & 6.22818046979039 & -0.53551953676852 & 7.28755121774513 \\
\hline $\mathrm{C}$ & 6.87578073381886 & -1.61486030139655 & 7.88240362836533 \\
\hline $\mathrm{C}$ & 6.63122985290801 & -2.89913378661740 & 7.41477740961725 \\
\hline $\mathrm{H}$ & 4.84652537767457 & 0.10192100937838 & 5.78753527881470 \\
\hline $\mathrm{H}$ & 6.41599874112175 & 0.46943192817629 & 7.64644159493278 \\
\hline $\mathrm{H}$ & 7.56390261445560 & -1.45243653507068 & 8.70242476407458 \\
\hline $\mathrm{C}$ & 5.75443766419698 & -3.09927347935603 & 6.35167458328091 \\
\hline $\mathrm{H}$ & 7.12723322136848 & -3.74591747959965 & 7.87246348242255 \\
\hline $\mathrm{H}$ & 5.58627841874296 & -4.10435946243308 & 5.98686470053507 \\
\hline C & -4.51592919466878 & -2.73840557337226 & 4.03502965288938 \\
\hline $\mathrm{C}$ & -3.91199118135936 & -2.89798951322542 & 6.44357225254908 \\
\hline $\mathrm{H}$ & -4.24642447261343 & -3.36167768842897 & 3.18008305717305 \\
\hline
\end{tabular}




\begin{tabular}{|llll|}
\hline$H$ & -4.69610426860339 & -1.72006983789498 & 3.66919535323664 \\
$H$ & -5.44535997140162 & -3.12454577103724 & 4.44563322549248 \\
$H$ & -3.89753645257084 & -1.93129944025204 & 6.96087876155719 \\
$H$ & -3.26950770841830 & -3.58654689809095 & 6.99348978927349 \\
$H$ & -4.92335324415105 & -3.29494989770135 & 6.48041563132306 \\
$H$ & 6.60998712260182 & -1.41170884270550 & 3.83307248971099 \\
$H$ & 1.65532552716068 & -0.77833054559217 & 1.82056776145053 \\
\hline
\end{tabular}




\begin{tabular}{|c|c|c|c|}
\hline \\
\hline \multicolumn{4}{|c|}{ BODIPY 35} \\
\hline $\mathrm{C}$ & -0.00413313217746 & -2.86497620406442 & 3.10061042578476 \\
\hline $\mathrm{N}$ & 0.75879474377856 & -3.07729231023547 & 4.25085856825163 \\
\hline C & 0.46085067643754 & -2.08590370674477 & 2.07287979990288 \\
\hline $\mathrm{C}$ & 1.72169245196530 & -1.51002243019936 & 2.09650020160141 \\
\hline B & 2.26487826195726 & -2.59249923003539 & 4.40905536108553 \\
\hline $\mathrm{C}$ & 3.76482771735187 & -1.11652571187252 & 2.86075729581928 \\
\hline $\mathrm{C}$ & 3.66475968680092 & -0.48044571138739 & 1.60253249886766 \\
\hline $\mathrm{C}$ & 2.36916281618621 & -0.71338123190874 & 1.12931107463432 \\
\hline C & 4.92213392079162 & -1.13313660250846 & 3.79078019261576 \\
\hline $\mathrm{H}$ & 4.71613591006435 & -0.50772855809250 & 4.66297021403499 \\
\hline $\mathrm{H}$ & 5.81398691894657 & -0.75105519638632 & 3.29852296170013 \\
\hline $\mathrm{H}$ & 5.11812186056509 & -2.13998967932763 & 4.16008687625689 \\
\hline $\mathrm{C}$ & 4.75423130526266 & 0.25457195528371 & 0.88409667312826 \\
\hline $\mathrm{C}$ & 4.84367542455583 & 1.75229674597628 & 1.20303668813009 \\
\hline $\mathrm{H}$ & 4.60491895363551 & 0.12887523462859 & -0.19171326870853 \\
\hline $\mathrm{H}$ & 5.71576222502362 & -0.21494707130600 & 1.10485136297282 \\
\hline $\mathrm{H}$ & 3.90519218218657 & 2.25728989117531 & 0.96815694417765 \\
\hline $\mathrm{H}$ & 5.63264158198345 & 2.22805410398667 & 0.61842092256645 \\
\hline $\mathrm{H}$ & 5.05868829317203 & 1.92090932556851 & 2.25987168502226 \\
\hline $\mathrm{C}$ & 1.72778470406432 & -0.17973694737320 & -0.11047717356122 \\
\hline $\mathrm{C}$ & 0.95982600718570 & 1.12855831920248 & 0.14784733966429 \\
\hline $\mathrm{H}$ & 1.04017778815427 & -0.92027245117956 & -0.52753418645288 \\
\hline $\mathrm{H}$ & 2.49020300080794 & -0.00868579370962 & -0.87296151278048 \\
\hline $\mathrm{H}$ & 0.16036073791968 & 0.97046238882932 & 0.87313337390448 \\
\hline $\mathrm{H}$ & 0.51417660813495 & 1.51252416538956 & -0.77210732282506 \\
\hline $\mathrm{H}$ & 1.62220535733102 & 1.89322304838868 & 0.55418681837732 \\
\hline $\mathrm{F}$ & 2.39625134717017 & -1.85015634057251 & 5.57772239188579 \\
\hline $\mathrm{F}$ & 3.06072160256073 & -3.73867886865981 & 4.46867884385211 \\
\hline $\mathrm{C}$ & -1.23179172262350 & -3.57845805612549 & 3.25609553809762 \\
\hline $\mathrm{C}$ & -1.16599553053681 & -4.20681596930648 & 4.54404213846921 \\
\hline $\mathrm{C}$ & 0.09135504645566 & -3.84315890348225 & 5.11711547027411 \\
\hline $\mathrm{C}$ & 0.58331562224983 & -4.19374551453676 & 6.47277688965193 \\
\hline $\mathrm{H}$ & 0.07026872191168 & -5.08019371527241 & 6.84203201013050 \\
\hline $\mathrm{H}$ & 0.37914458470061 & -3.37207182571527 & 7.16505338209269 \\
\hline $\mathrm{H}$ & 1.65757170599648 & -4.36788860757093 & 6.47600339745305 \\
\hline $\mathrm{C}$ & -2.33137908558417 & -3.73880172060668 & 2.42820444691805 \\
\hline $\mathrm{C}$ & -3.36766074653880 & -4.53324227710316 & 2.90769465990732 \\
\hline $\mathrm{C}$ & -2.19959337215752 & -5.00783911836469 & 5.02037535233386 \\
\hline $\mathrm{C}$ & -4.63277473721056 & -4.87876876288842 & 2.34152880635449 \\
\hline $\mathrm{N}$ & -5.29596531300013 & -5.65271997221770 & 3.20264066973029 \\
\hline $\mathrm{C}$ & -4.52397786625115 & -5.89085353059332 & 4.34180041125361 \\
\hline $\mathrm{C}$ & -3.29752230177209 & -5.17240574848797 & 4.19078342946694 \\
\hline $\mathrm{C}$ & -5.13909633917641 & -4.49715255624867 & 0.99943272506640 \\
\hline $\mathrm{H}$ & -4.61473667013658 & -3.61483841223052 & 0.63615333924965 \\
\hline $\mathrm{H}$ & -6.20965809270401 & -4.30167971468400 & 1.01568111375051 \\
\hline $\mathrm{H}$ & -4.96389821946539 & -5.31125269189011 & 0.29064337574946 \\
\hline $\mathrm{C}$ & -4.97266351744510 & -6.71840550466392 & 5.33963329542054 \\
\hline $\mathrm{C}$ & -6.22459185644118 & -7.31495991573381 & 5.29713588085288 \\
\hline $\mathrm{H}$ & -4.32968410938364 & -6.91440378176912 & 6.18600568829335 \\
\hline $\mathrm{H}$ & -2.38423246302309 & -3.26866161314993 & 1.45513300046595 \\
\hline $\mathrm{H}$ & -2.14757830427941 & -5.47651796692543 & 5.99418819596329 \\
\hline
\end{tabular}




\begin{tabular}{|llll|}
\hline N & 2.59489813976699 & -1.73410465551563 & 3.15375629663811 \\
N & -7.10806748870837 & -7.05575499421643 & 4.25706686736434 \\
C & -8.26555689789214 & -7.70674987632254 & 4.52283208147493 \\
C & -8.14712021048825 & -8.40397022418891 & 5.74665604359669 \\
C & -6.85252824225133 & -8.17240009438759 & 6.22492497081180 \\
C & -6.19614195805534 & -8.75992076355724 & 7.43226055833437 \\
C & -5.42170196373825 & -10.04939523633873 & 7.10875617704970 \\
H & -6.95086166402599 & -8.97232472200131 & 8.19200177961853 \\
H & -5.50992378231360 & -8.03427848690642 & 7.87734246822358 \\
H & -6.08203283255201 & -10.80067260485684 & 6.67507074143491 \\
H & -4.96449187204545 & -10.46983767026793 & 8.00674730588170 \\
H & -4.63055438349462 & -9.85327109724867 & 6.38375623601611 \\
C & -9.22227920111112 & -9.18916519846125 & 6.43309482760798 \\
C & -9.29510927379010 & -10.67150706650836 & 6.04458386636942 \\
H & -10.19090804603472 & -8.72205659321601 & 6.23967023400464 \\
H & -9.06896408769795 & -9.11347208011802 & 7.51301323467570 \\
H & -9.51046341334492 & -10.79330431673625 & 4.98119204772501 \\
H & -10.07761849261950 & -11.18271213601536 & 6.60737103053010 \\
H & -8.35041651863915 & -11.17588789731015 & 6.25450053046186 \\
C & -9.42641966657484 & -7.66047435507578 & 3.59810339348427 \\
H & -10.31328736347377 & -8.07240790704354 & 4.07493770749819 \\
H & -9.21677858388485 & -8.24479661150584 & 2.69873321312992 \\
H & -9.63385950113695 & -6.64057713458285 & 3.27346000634002 \\
B & -6.81165028541459 & -6.10418821160453 & 3.06615334576786 \\
F & -7.59097264403999 & -4.94648905068640 & 3.13238334459741 \\
F & -6.99691011148680 & -6.74946768472418 & 1.84841945219198 \\
H & -0.17262404035341 & -1.92037858390156 & 1.21307397231650 \\
\hline
\end{tabular}




\begin{tabular}{|c|c|c|c|}
\hline 78 & & & \\
\hline & DIPY 36 & & \\
\hline $\mathrm{C}$ & 0.67460963495409 & -3.86244273708301 & 4.57183185936034 \\
\hline $\mathrm{C}$ & 0.58942161476393 & -4.58373355543426 & 5.81407585602351 \\
\hline C & -0.38470804487857 & -3.82119077847469 & 3.67550993734620 \\
\hline $\mathrm{C}$ & -1.53928444200029 & -4.50390419530842 & 4.03266640478880 \\
\hline $\mathrm{C}$ & -0.56901156224806 & -5.27312027404374 & 6.16244133061587 \\
\hline C & -2.80000986741082 & -4.62786380334612 & 3.37234326776679 \\
\hline $\mathrm{N}$ & -3.61709425306709 & -5.35354790908162 & 4.13095333836563 \\
\hline $\mathrm{C}$ & -2.96206195900863 & -5.77115778280757 & 5.30143899325623 \\
\hline C & -1.63711407086939 & -5.22788193277927 & 5.27142319175278 \\
\hline $\mathrm{C}$ & -3.15403552465545 & -4.07827450346820 & 2.03971337585016 \\
\hline $\mathrm{H}$ & -2.54116468772852 & -3.20596094120854 & 1.81658643323736 \\
\hline $\mathrm{H}$ & -4.20546374174710 & -3.80621088148727 & 1.98224496428143 \\
\hline $\mathrm{H}$ & -2.96532733181307 & -4.82988524725630 & 1.26822628683782 \\
\hline $\mathrm{C}$ & -3.60086545402188 & -6.59239124021285 & 6.19940534155562 \\
\hline $\mathrm{C}$ & -4.90637669907767 & -7.03542077918381 & 6.00917639935663 \\
\hline $\mathrm{H}$ & -3.06575906614756 & -6.91593640623479 & 7.08133491122648 \\
\hline $\mathrm{H}$ & -0.31585535593360 & -3.27198721149810 & 2.74579534220637 \\
\hline $\mathrm{H}$ & -0.64575397804327 & -5.80731308324310 & 7.10078381531581 \\
\hline $\mathrm{N}$ & -5.64115866257448 & -6.63111508196654 & 4.90070296489744 \\
\hline $\mathrm{C}$ & -6.86867311509181 & -7.19277016670238 & 4.98001159694797 \\
\hline C & -6.95287992644029 & -7.98198173978083 & 6.14750514323891 \\
\hline $\mathrm{C}$ & -5.71525278585478 & -7.88921479917181 & 6.79454656346247 \\
\hline $\mathrm{C}$ & -5.28248817027377 & -8.59268201868224 & 8.03861001464963 \\
\hline $\mathrm{C}$ & -4.64003536724397 & -9.96291248197682 & 7.76158333501787 \\
\hline $\mathrm{H}$ & -6.14351551268545 & -8.72478886758771 & 8.69791707048574 \\
\hline $\mathrm{H}$ & -4.57219212387660 & -7.97013935800529 & 8.58816870099713 \\
\hline $\mathrm{H}$ & -5.34746183531764 & -10.63346722713845 & 7.27355797702524 \\
\hline $\mathrm{H}$ & -4.30541987404628 & -10.43242352679327 & 8.68845232749336 \\
\hline $\mathrm{H}$ & -3.77809552874209 & -9.86037533809733 & 7.10054139995643 \\
\hline $\mathrm{C}$ & -8.15955649300473 & -8.74592027459695 & 6.60155470229860 \\
\hline $\mathrm{C}$ & -8.27495005309609 & -10.15896400772610 & 6.01080184596886 \\
\hline $\mathrm{H}$ & -9.06037332417392 & -8.18051860895498 & 6.35109018671430 \\
\hline $\mathrm{H}$ & -8.14771577019264 & -8.81544562731161 & 7.69236799875926 \\
\hline $\mathrm{H}$ & -8.32425029205293 & -10.12879553327054 & 4.92071559027878 \\
\hline $\mathrm{H}$ & -9.17169547126313 & -10.66278654751724 & 6.37726884587918 \\
\hline $\mathrm{H}$ & -7.41300917566293 & -10.76831579104097 & 6.28605127334264 \\
\hline $\mathrm{C}$ & -7.91175110388256 & -6.96600218435495 & 3.94632031762839 \\
\hline $\mathrm{H}$ & -8.87087367196240 & -7.35752309020205 & 4.27894510677759 \\
\hline $\mathrm{H}$ & -7.63978865516840 & -7.46302328856716 & 3.01238490913096 \\
\hline $\mathrm{H}$ & -8.01874065992558 & -5.90428163111599 & 3.72067200427639 \\
\hline B & -5.15784374185835 & -5.60071339362953 & 3.85162655619626 \\
\hline $\mathrm{F}$ & -5.82556114291666 & -4.38378606794560 & 4.00393662027274 \\
\hline $\mathrm{F}$ & -5.31746527839346 & -6.08341596050395 & 2.55750615444096 \\
\hline C & 1.96021255207540 & -3.24343803859194 & 4.53117665896521 \\
\hline $\mathrm{N}$ & 2.61871261632623 & -3.54512574356623 & 5.64583449350570 \\
\hline $\mathrm{C}$ & 1.84297168263000 & -4.36638661724723 & 6.47693313542232 \\
\hline $\mathrm{C}$ & 2.52360948409274 & -2.41096186365913 & 3.44015673612418 \\
\hline $\mathrm{H}$ & 3.17663801741468 & -1.63743301907571 & 3.83710455741576 \\
\hline $\mathrm{H}$ & 1.72361798928244 & -1.94912221356640 & 2.86297506306977 \\
\hline $\mathrm{H}$ & 3.11965149872120 & -3.03187318929624 & 2.76565187586492 \\
\hline $\mathrm{N}$ & 4.38241351656008 & -3.57291071705943 & 7.43426121580146 \\
\hline
\end{tabular}




\begin{tabular}{|llll|}
\hline B & 4.05717798272985 & -3.02421184879506 & 6.02493762921160 \\
F & 4.02501193839912 & -1.62695548184989 & 6.03887412993156 \\
F & 4.97510882769303 & -3.47462470155269 & 5.07749962885112 \\
C & 2.31655744602339 & -4.78147374221722 & 7.69951508310671 \\
C & 3.56230834702274 & -4.40135615525702 & 8.20076012676944 \\
H & 1.67904403875857 & -5.41278211849330 & 8.29612016395639 \\
C & 5.49856550854653 & -3.29864195062518 & 8.14189241420020 \\
C & 4.22049505360748 & -4.64733781743257 & 9.43684455613639 \\
C & 5.43888984618083 & -3.95456871556320 & 9.38588101185647 \\
C & 6.57806082286116 & -2.42203843624802 & 7.61314881289554 \\
H & 6.17667831133517 & -1.67426634836765 & 6.93388672988473 \\
H & 7.31765562768931 & -3.00915619232164 & 7.06210557302810 \\
H & 7.09156993459699 & -1.91649651922565 & 8.42995941364654 \\
C & 3.77131010182120 & -5.43309676283345 & 10.63923556359558 \\
C & 2.42259222813200 & -6.14500513330124 & 10.59418964435919 \\
H & 3.77043199690169 & -4.75446061249117 & 11.49877283686822 \\
H & 4.54475492123812 & -6.17375474553397 & 10.86674503742248 \\
H & 1.59879783933667 & -5.43841758324443 & 10.48206366496748 \\
H & 2.26279650265473 & -6.68817528987988 & 11.52698027014297 \\
H & 2.37153410860846 & -6.87298416937018 & 9.78302889560303 \\
C & 6.47450140413546 & -3.86823779250345 & 10.47019906898978 \\
C & 7.89636061070529 & -4.26940079908760 & 10.05558552081935 \\
H & 6.16496792141521 & -4.49655264774151 & 11.30662239331257 \\
H & 6.50164913633200 & -2.84595055749664 & 10.86486214629549 \\
H & 7.92542148822104 & -5.29807551219667 & 9.69075765398295 \\
H & 8.57427709579733 & -4.18443689801298 & 10.90737810605586 \\
H & 8.28596612678773 & -3.63183419150343 & 9.26463993266005 \\
\hline
\end{tabular}




\begin{tabular}{|clll|}
\hline \multicolumn{2}{|l}{ R } & & \\
BODIPY 37 & & \\
C & 0.51184388715060 & -4.38274309132954 & 4.67859431070486 \\
C & 0.54739113202177 & -5.26142410190156 & 5.74709013037835 \\
C & 1.63177470460813 & -3.54275235846422 & 4.80513755077464 \\
N & 2.33939811524090 & -3.88206759667005 & 5.88674455067434 \\
C & 1.70609310041921 & -4.95453538143527 & 6.50609044617097 \\
N & 4.09054928638787 & -3.93814991895653 & 7.64296072038769 \\
B & 3.58261451097312 & -3.10702623127932 & 6.42485947165460 \\
F & 3.20917105421409 & -1.83199460832981 & 6.82672845920979 \\
F & 4.56763071596217 & -3.05210175177351 & 5.45042102227505 \\
C & 2.24749402621157 & -5.52680764114782 & 7.66919980870907 \\
C & 3.46168674822900 & -5.04367062493174 & 8.19768919922806 \\
C & 1.56726039158437 & -6.60159752286766 & 8.35332603917511 \\
C & 5.25246216115127 & -3.74737217101657 & 8.28215405624618 \\
C & 4.30301397783344 & -5.54585247733253 & 9.21921740638260 \\
C & 5.42125041309099 & -4.72697746338729 & 9.27260350332450 \\
H & 1.94442421574273 & -2.71999299948811 & 4.18313232728972 \\
H & 5.91066207340049 & -2.94442689517202 & 7.99354205179150 \\
H & -0.22736000646818 & -4.33774829310219 & 3.89683695169348 \\
H & -0.15276455385906 & -6.04710314049421 & 5.96143124393810 \\
H & 4.12858874671971 & -6.43360773338818 & 9.80022640414449 \\
H & 6.27422048296094 & -4.82983526393667 & 9.92188838989512 \\
C & 1.47783034812504 & -6.96052097076888 & 9.67267488126164 \\
O & 0.77063606672469 & -7.44967082105499 & 7.61306003797308 \\
C & 0.20794956621435 & -8.32212197847064 & 8.47262828067094 \\
C & 0.59768810553481 & -8.07428030760434 & 9.75317667484251 \\
Br & -0.91729494825758 & -9.60925203968036 & 7.74013759583012 \\
H & 1.95118934037892 & -6.45126627391601 & 10.49333641546474 \\
H & 0.28659633770467 & -8.61062034209998 & 10.63184206990874 \\
\hline
\end{tabular}




\begin{tabular}{|c|c|c|c|}
\hline 46 & & & \\
\hline & DIPY 38 & & \\
\hline $\mathrm{C}$ & -0.30063985306276 & -4.55105369823246 & 4.59820527419426 \\
\hline $\mathrm{C}$ & -0.21220440675430 & -5.38723778452270 & 5.68496590533491 \\
\hline C & 0.88963888656580 & -3.75746329356925 & 4.59502962058791 \\
\hline $\mathrm{N}$ & 1.65318128889882 & -4.09536166088450 & 5.65083716699280 \\
\hline $\mathrm{C}$ & 1.01842570269997 & -5.12282757690710 & 6.34643874755322 \\
\hline $\mathrm{N}$ & 3.55411393997986 & -4.23165771927360 & 7.23707628790317 \\
\hline B & 2.98035113261400 & -3.39483293101776 & 6.05679350011778 \\
\hline $\mathrm{F}$ & 2.73944972122746 & -2.08147710713353 & 6.44362983467786 \\
\hline $\mathrm{F}$ & 3.82190372555798 & -3.40480913422851 & 4.93218367063543 \\
\hline C & 1.60907999121486 & -5.70838424937628 & 7.44800772325181 \\
\hline $\mathrm{C}$ & 2.87455804214415 & -5.26552387200793 & 7.88420781505395 \\
\hline $\mathrm{C}$ & 0.93475796774588 & -6.82017399126177 & 8.15260467044814 \\
\hline $\mathrm{C}$ & 4.74252526725307 & -4.09157153557186 & 7.85978528153630 \\
\hline $\mathrm{C}$ & 3.69321739301668 & -5.75045087964644 & 8.91745010373225 \\
\hline $\mathrm{C}$ & 4.87216564381369 & -5.00981944224934 & 8.90435721676846 \\
\hline $\mathrm{Cl}$ & 5.89676825822174 & -2.93248706616148 & 7.37276796416844 \\
\hline $\mathrm{H}$ & -1.07771536201198 & -4.51964839915491 & 3.85632580297073 \\
\hline $\mathrm{H}$ & -0.92577972591895 & -6.13438138783594 & 5.98771676041551 \\
\hline $\mathrm{C}$ & 0.54548309610450 & -6.69516571932012 & 9.48745541275805 \\
\hline $\mathrm{C}$ & 0.66517107187895 & -8.01904063533027 & 7.49081754072982 \\
\hline C & 0.02290236052534 & -9.06034205111492 & 8.14645106471106 \\
\hline $\mathrm{C}$ & -0.37052852221003 & -8.94020210699067 & 9.47836930999698 \\
\hline C & -0.10118928322946 & -7.73779413937961 & 10.13340727968897 \\
\hline $\mathrm{H}$ & 0.73122916988183 & -5.76685863787659 & 10.01129931935430 \\
\hline C & -1.04362625454382 & -10.07569352462635 & 10.19702650847746 \\
\hline $\mathrm{H}$ & -0.31545129588981 & -10.66658842137325 & 10.76066663288460 \\
\hline $\mathrm{H}$ & -1.54292664170062 & -10.74876573229443 & 9.49913316420015 \\
\hline $\mathrm{H}$ & -1.78452145334239 & -9.70964107825404 & 10.90876434823257 \\
\hline $\mathrm{N}$ & 1.32797696902053 & -2.83910591663706 & 3.70837716047899 \\
\hline C & 0.61166778069226 & -2.20385220838581 & 2.68809263083886 \\
\hline C & -0.75770531091295 & -1.95338798546626 & 2.77889071087980 \\
\hline C & -1.40811660240334 & -1.29300484473851 & 1.74529556542752 \\
\hline C & -0.70795598229416 & -0.86324014668521 & 0.62420659438883 \\
\hline C & 0.66179677894203 & -1.09313484946412 & 0.54728877606501 \\
\hline C & 1.31868154683034 & -1.76425991727765 & 1.56585337846216 \\
\hline $\mathrm{H}$ & -1.30021260978976 & -2.23697430719563 & 3.66888171556627 \\
\hline $\mathrm{H}$ & -2.46834986863020 & -1.09335281129640 & 1.83102213100267 \\
\hline $\mathrm{H}$ & -1.22037828833064 & -0.34276524206147 & -0.17310985774750 \\
\hline $\mathrm{H}$ & 1.22310776717667 & -0.75239534610966 & -0.31277667534171 \\
\hline $\mathrm{H}$ & 2.38200116999402 & -1.95970884228074 & 1.49842770075931 \\
\hline $\mathrm{H}$ & 2.31039594565957 & -2.60917828546630 & 3.78799539146179 \\
\hline $\mathrm{H}$ & 0.97711574926526 & -8.13753841887345 & 6.46150155763235 \\
\hline $\mathrm{H}$ & -0.17453301878031 & -9.98289985915141 & 7.61319336122410 \\
\hline $\mathrm{H}$ & -0.41471375923879 & -7.61147384597843 & 11.16261972394950 \\
\hline $\mathrm{H}$ & 3.44230318703315 & -6.56261837106027 & 9.57815491565344 \\
\hline $\mathrm{H}$ & 5.72994868508587 & -5.10718502627601 & 9.54664129192173 \\
\hline
\end{tabular}




\begin{tabular}{|c|c|c|c|}
\hline 58 & & & \\
\hline & DIPY 39 & & \\
\hline $\mathrm{C}$ & -0.08796594957701 & -4.17330297497403 & 4.73861205763323 \\
\hline $\mathrm{C}$ & -0.02985937569066 & -5.02220641288844 & 5.83020442457142 \\
\hline C & 1.06024878335776 & -3.34363540906320 & 4.81040196192014 \\
\hline $\mathrm{N}$ & 1.77547469697904 & -3.65686119011886 & 5.91230944247613 \\
\hline $\mathrm{C}$ & 1.13813503120375 & -4.71929579564737 & 6.56142113059616 \\
\hline $\mathrm{N}$ & 3.59965376113020 & -3.79230816971934 & 7.57471911031625 \\
\hline B & 3.04478367414836 & -2.94272849658936 & 6.41394365679818 \\
\hline $\mathrm{F}$ & 2.77092089867439 & -1.63437864789689 & 6.80697529418718 \\
\hline $\mathrm{F}$ & 4.00385047533880 & -2.88707678206377 & 5.36109887492534 \\
\hline C & 1.70085664872459 & -5.31587996943804 & 7.68712593316536 \\
\hline $\mathrm{C}$ & 2.91117743422184 & -4.84329330352399 & 8.19352948249705 \\
\hline $\mathrm{C}$ & 1.11040920359911 & -6.54425868471787 & 8.26444958345048 \\
\hline $\mathrm{C}$ & 4.82277498927675 & -3.69820185633928 & 8.14431332658445 \\
\hline $\mathrm{C}$ & 3.74657275159281 & -5.35864526555044 & 9.20676704801043 \\
\hline $\mathrm{C}$ & 4.93253515522563 & -4.64763358672683 & 9.19129552578760 \\
\hline $\mathrm{N}$ & 5.75842514995542 & -2.84747423058299 & 7.64496410753400 \\
\hline $\mathrm{H}$ & -0.82580588598369 & -4.15733244122030 & 3.95658214061868 \\
\hline $\mathrm{H}$ & -0.73188298331402 & -5.79782544350531 & 6.08523679855881 \\
\hline $\mathrm{H}$ & 3.50049512571188 & -6.19077037628657 & 9.84400245367666 \\
\hline $\mathrm{H}$ & 5.80318929735926 & -4.81092066536851 & 9.80006927757367 \\
\hline C & 0.68271966422585 & -6.61488376870909 & 9.58946776164666 \\
\hline $\mathrm{C}$ & 1.06389645022927 & -7.70535838559721 & 7.48945856331237 \\
\hline $\mathrm{C}$ & 0.64004300994394 & -8.90550456826039 & 8.03633600138412 \\
\hline $\mathrm{C}$ & 0.24288859602563 & -8.99075234728517 & 9.36985635085924 \\
\hline $\mathrm{C}$ & 0.25766937338612 & -7.82166883802053 & 10.13058190448939 \\
\hline $\mathrm{H}$ & 0.69180104657951 & -5.72123140083855 & 10.19973303498763 \\
\hline $\mathrm{C}$ & -0.19059656521928 & -10.30948287870547 & 9.94864459927297 \\
\hline $\mathrm{H}$ & 0.33314894087016 & -11.13597513966660 & 9.46830856763844 \\
\hline $\mathrm{H}$ & -1.26195948643544 & -10.47226161518507 & 9.79757853191699 \\
\hline $\mathrm{H}$ & 0.00935248574023 & -10.35957527995806 & 11.02011974175620 \\
\hline $\mathrm{N}$ & 1.51028367541308 & -2.40274013028524 & 3.94020414996690 \\
\hline C & 0.82788990679498 & -1.95188812578503 & 2.80017655856346 \\
\hline $\mathrm{C}$ & -0.51973240169848 & -1.59529454048242 & 2.85874752391474 \\
\hline C & -1.16827966152466 & -1.15271611179928 & 1.71510785947276 \\
\hline $\mathrm{C}$ & -0.48057532136320 & -1.03259808713433 & 0.51252516030084 \\
\hline $\mathrm{C}$ & 0.86898738893930 & -1.36264332319509 & 0.46147962726769 \\
\hline $\mathrm{C}$ & 1.52047458931810 & -1.82710856230127 & 1.59475610432231 \\
\hline $\mathrm{H}$ & -1.04397617239392 & -1.64567109365611 & 3.80285864295477 \\
\hline $\mathrm{H}$ & -2.21293173541696 & -0.87608184598861 & 1.77146631828053 \\
\hline $\mathrm{H}$ & -0.98945227692313 & -0.67968306147558 & -0.37420818713723 \\
\hline $\mathrm{H}$ & 1.41654868379047 & -1.27223590227918 & -0.46776456755756 \\
\hline $\mathrm{H}$ & 2.56314555607491 & -2.11577580816737 & 1.54807674840222 \\
\hline $\mathrm{H}$ & 2.49008299411666 & -2.17120652584276 & 4.02020036450577 \\
\hline $\mathrm{H}$ & 1.40533449345839 & -7.66821875232135 & 6.46381428168321 \\
\hline $\mathrm{H}$ & 0.63993999709518 & -9.79929320445494 & 7.42381689222673 \\
\hline $\mathrm{H}$ & -0.06168012589488 & -7.85366625726756 & 11.16525876310846 \\
\hline $\mathrm{C}$ & 7.05830957801647 & -2.68356286361261 & 8.14943326309355 \\
\hline $\mathrm{C}$ & 7.28676965911303 & -2.51136531455860 & 9.51465445566819 \\
\hline C & 8.14004099716717 & -2.67275295793463 & 7.26684913972791 \\
\hline $\mathrm{C}$ & 9.43170367736393 & -2.50939350600817 & 7.74805914184190 \\
\hline $\mathrm{C}$ & 9.66188601219968 & -2.36782175495895 & 9.11137470363023 \\
\hline
\end{tabular}




\begin{tabular}{|lllc|}
\hline C & 8.58295332802415 & -2.36680803121444 & 9.98847753995432 \\
H & 7.96462792880973 & -2.81679611772821 & 6.20780759086175 \\
H & 6.44709613776606 & -2.47108789505145 & 10.19398413347466 \\
H & 8.74890185060726 & -2.23397981737057 & 11.04996232514855 \\
H & 10.26248194460644 & -2.51082299790810 & 7.05450246413712 \\
H & 10.66975780295521 & -2.25231857440040 & 9.48674733390000 \\
H & 5.58230909630478 & -2.52557491237030 & 6.70351498014095 \\
\hline
\end{tabular}




\begin{tabular}{|c|c|c|c|}
\hline \\
\hline \multicolumn{4}{|c|}{ BODIPY 40} \\
\hline & 0.22367465972151 & -5.11328562092133 & 4.7731682521634 \\
\hline & 0.30697871994301 & -5.89853796253113 & 5.91236453384663 \\
\hline & 1.52016121376564 & -4.57651567279920 & 541594 \\
\hline & 2.36542480181657 & 32515081 & 001020 \\
\hline & 1.64751338408243 & -5.82128292323124 & 212140 \\
\hline $\mathrm{N}$ & 4.27957843982914 & -5.17060506011496 & 4751 \\
\hline & 3.88897625549720 & -4.59402378291997 & 5.74941037214877 \\
\hline & 3.98465569118529 & -3.2195 & $5.7 \varepsilon$ \\
\hline & 9129249231 & & \\
\hline & 2.23938819 & -6.40 & 185 \\
\hline & 3.52671712012566 & 843096635781 & 7419 \\
\hline & 5.40468319587654 & -4.95931418086655 & 12265 \\
\hline & 4.25794753040322 & -6.51840233025576 & 19864232981 \\
\hline & 954414 & 398 & 0363 \\
\hline & 19 & -3. & \\
\hline $\mathrm{C}$ & 325 & & 211 \\
\hline $\mathrm{O}$ & 6517 & -3.1 & 5357 \\
\hline U & 7608 & 13 & 9602 \\
\hline 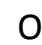 & 1535 & & 5923 \\
\hline U & 6.83 & 8992 & 152 \\
\hline$c$ & -1.0 & 810 & 821 \\
\hline $\mathrm{C}$ & 6724 & 9775 & 3792 \\
\hline $\mathrm{H}$ & -0.86692593886695 & 0777 & 35742 \\
\hline $\mathrm{H}$ & -1.43218476820723 & 007 & 587 \\
\hline C & -2.1739 & 38437 & 996 \\
\hline C & -0.8 & 1481 & 914 \\
\hline $\mathrm{H}$ & 5263 & 8798 & 296 \\
\hline $\mathrm{H}$ & 6571 & 4286 & 210 \\
\hline$c$ & 877 & -7.5 & 666 \\
\hline C & 5.15452372685638 & 344321 & 16233 \\
\hline $\mathrm{H}$ & 3.39026880830131 & 886 & 041 \\
\hline $\mathrm{H}$ & 3.1 & -7 & 197 \\
\hline $\mathrm{C}$ & 5.9 & -6 . & 5091 \\
\hline C & 6.51 & -5 & 3319 \\
\hline & 7.36412 & -6.4 & 9470 \\
\hline $\mathrm{H}$ & 6.88102689295345 & -4.9 & 6065 \\
\hline $\mathrm{H}$ & -1.8 & 177 & 5644 \\
\hline $\mathrm{H}$ & $-3 . c$ & 355 & 5080 \\
\hline $\mathrm{H}$ & -2.4 & 369 & 9570 \\
\hline & -2.98712099993144 & -6.77292739367368 & 820690 \\
\hline $\mathrm{H}$ & 5.39286073240126 & -6.1371698 & 21274 \\
\hline $\mathrm{H}$ & 6.84 & & \\
\hline $\mathrm{H}$ & 5.75 & -8 & 10. \\
\hline $\mathrm{H}$ & 4.87 & -8. & 11.6 \\
\hline C & 64867 & -2.33 & 1329 \\
\hline & $7.7 \varepsilon$ & -2.21 & 8470 \\
\hline$\Pi$ & 0.37 & -1.66 & 6168 \\
\hline & & & \\
\hline & 2.12391011792 & -1.75485924223998 & 1.87170057890838 \\
\hline & 0.50941356049297 & -3.76413947293252 & 0.20413218468302 \\
\hline & 2.25791677734873 & -3.83488792704108 & 0.4977690512558 \\
\hline
\end{tabular}




\begin{tabular}{|llll|}
\hline H & 1.57332722554055 & -2.50886920875258 & -0.44919738380888 \\
C & 8.08825837394010 & -1.58997091790421 & 9.69510875056221 \\
H & 7.35564303179744 & -1.49874242891789 & 7.65090948660779 \\
H & 8.67430032380497 & -2.64582061663336 & 7.88459804701746 \\
H & 7.17890531152779 & -1.19819372020408 & 10.15232322407033 \\
H & 8.78998728991848 & -0.76382269870003 & 9.57164369893277 \\
H & 8.53516048861957 & -2.31738277774364 & 10.37381836296350 \\
H & 1.65312961698781 & -7.08723169466909 & 8.08184591756300 \\
\hline
\end{tabular}




\begin{tabular}{|c|c|c|c|}
\hline 51 & & & \\
\hline & DIPY 41 & & \\
\hline $\mathrm{C}$ & 0.17805821629208 & -5.07168224593530 & 4.78609888088245 \\
\hline $\mathrm{C}$ & 0.27002371799356 & -5.91698462931743 & 5.92548253029124 \\
\hline C & 1.48656823066009 & -4.53926481150150 & 4.57299725336773 \\
\hline $\mathrm{N}$ & 2.31833658374576 & -4.99614998052945 & 5.53095650907697 \\
\hline $\mathrm{C}$ & 1.62466461211566 & -5.84548771562513 & 6.37304548255025 \\
\hline $\mathrm{N}$ & 4.27075374684202 & -5.19020061740162 & 7.19416621503238 \\
\hline B & 3.85925123381771 & -4.60414445923051 & 5.75482237799302 \\
\hline $\mathrm{F}$ & 3.95096487869790 & -3.23605798017221 & 5.79777253984474 \\
\hline $\mathrm{F}$ & 4.62943519760289 & -5.25206494767522 & 4.83492237134970 \\
\hline $\mathrm{C}$ & 2.22780261534490 & -6.42859137204011 & 7.47509039314729 \\
\hline $\mathrm{C}$ & 3.51090098892465 & -6.12712863361521 & 7.87889666974311 \\
\hline $\mathrm{C}$ & 5.39835901877820 & -4.94791992211677 & 7.87545954520930 \\
\hline $\mathrm{C}$ & 4.24053727056572 & -6.52999870217694 & 9.04213133408474 \\
\hline $\mathrm{C}$ & 5.43586146542609 & -5.76275895122784 & 9.04869048525401 \\
\hline $\mathrm{C}$ & 6.43433446282431 & -3.97439438017286 & 7.43923537148974 \\
\hline $\mathrm{C}$ & 1.94497950077661 & -3.68551088141429 & 3.45334351669730 \\
\hline 0 & 0.89121978862547 & -3.08810687847772 & 2.85237283216119 \\
\hline 0 & 3.08682296579405 & -3.55274346322188 & 3.10467387123338 \\
\hline 0 & 6.96348255895410 & -3.37485241162819 & 8.52614913942062 \\
\hline 0 & 6.77156068635375 & -3.75858852471097 & 6.30878446986665 \\
\hline C & -1.05330524447307 & -4.90169164592747 & 4.13204702371439 \\
\hline $\mathrm{C}$ & -2.15572826540846 & -5.55887089017766 & 4.63407062481920 \\
\hline $\mathrm{C}$ & -2.06036138932201 & -6.39582814758710 & 5.76437210424768 \\
\hline $\mathrm{C}$ & -0.85773902781929 & -6.58480594855726 & 6.41354268840586 \\
\hline $\mathrm{C}$ & 3.98718141072868 & -7.45647938982504 & 10.05659842728647 \\
\hline $\mathrm{C}$ & 4.93141951724320 & -7.60664208964249 & 11.05334681028793 \\
\hline $\mathrm{C}$ & 6.11980302859172 & -6.85010008529631 & 11.05902483128080 \\
\hline $\mathrm{C}$ & 6.38242069633497 & -5.92489945656081 & 10.07013432362294 \\
\hline C & 1.19834591304896 & -2.22822959173462 & 1.73135148479916 \\
\hline $\mathrm{C}$ & 7.97087740172391 & -2.37031759333428 & 8.27559190587174 \\
\hline $\mathrm{H}$ & 0.34672003214118 & -1.55190026398540 & 1.67639022921956 \\
\hline C & 1.36903283560193 & -3.01442578169044 & 0.44691909785882 \\
\hline $\mathrm{H}$ & 2.09617797043959 & -1.65854851528506 & 1.96678696872783 \\
\hline $\mathrm{H}$ & 0.47203580854876 & -3.59345194153142 & 0.22120070161792 \\
\hline $\mathrm{H}$ & 2.21895141736270 & -3.69167737648770 & 0.51615402151615 \\
\hline $\mathrm{H}$ & 1.54873160510787 & -2.32276550812867 & -0.37861198140811 \\
\hline $\mathrm{C}$ & 8.35642651111353 & -1.77698286131672 & 9.61227923259426 \\
\hline $\mathrm{H}$ & 7.55138432089224 & -1.62476277076285 & 7.59940593189888 \\
\hline $\mathrm{H}$ & 8.81503352843278 & -2.84142278742678 & 7.76969678755322 \\
\hline $\mathrm{H}$ & 7.48721605300631 & -1.34146714440410 & 10.10631278145255 \\
\hline $\mathrm{H}$ & 9.09697151161944 & -0.98900022360400 & 9.46808950757460 \\
\hline $\mathrm{H}$ & 8.78852795085936 & -2.53402180786526 & 10.26774369593658 \\
\hline $\mathrm{H}$ & 1.64696191502214 & -7.11622648677157 & 8.07454560692446 \\
\hline $\mathrm{H}$ & 6.83679658522717 & -7.00331823266916 & 11.85469118105274 \\
\hline $\mathrm{H}$ & 7.28878335449260 & -5.33833438812723 & 10.07748426044962 \\
\hline $\mathrm{H}$ & 3.08234066331286 & -8.05017812331771 & 10.06141798515060 \\
\hline $\mathrm{H}$ & 4.75783605093002 & -8.32306233271803 & 11.84565562209166 \\
\hline $\mathrm{H}$ & -2.94802384801262 & -6.89544269782682 & 6.12995902440448 \\
\hline $\mathrm{H}$ & -0.79753842498723 & -7.22548169205990 & 7.28353045911156 \\
\hline $\mathrm{H}$ & -1.13185367137964 & -4.25819216431290 & 3.27040615971846 \\
\hline $\mathrm{H}$ & -3.11683395051514 & -5.42811055287408 & 4.15451071352219 \\
\hline
\end{tabular}




\begin{tabular}{|llll|}
\hline BO & & & \\
BODIPY 42 & & \\
N & 2.54847558774105 & -4.67659209242540 & 5.71462193598233 \\
C & 2.01831491536881 & -5.37782365440955 & 6.71616226770462 \\
N & 4.09383029071509 & -4.92164643726409 & 7.50830339244838 \\
B & 4.06094962151407 & -4.19480804843757 & 6.10419616506615 \\
F & 4.09600120716946 & -2.82366799285834 & 6.20640441910845 \\
F & 4.97992723486684 & -4.68211824442024 & 5.20483535127740 \\
C & 2.91066115897599 & -5.53019999671233 & 7.81445285977257 \\
C & 4.89004468328111 & -5.11457050402760 & 8.60484935471049 \\
C & 4.11916303735179 & -5.82959687353358 & 9.52329678634085 \\
N & 2.87555133400160 & -6.08665918977723 & 9.00443919537259 \\
N & 0.77299565961115 & -5.73946875662247 & 6.39042752081125 \\
C & 0.48328538050301 & -5.23808053036247 & 5.11588872704830 \\
C & 1.60682929243516 & -4.57333630189002 & 4.70559488525067 \\
H & 0.16207792211250 & -6.28276296266164 & 6.97629463852277 \\
C & 6.30102397017731 & -4.64869091612501 & 8.69387243439345 \\
C & 4.47698225090326 & -6.26639445724106 & 10.90349606122141 \\
H & 6.82125174953581 & -4.81063799399891 & 7.74831380225775 \\
H & 6.83280888777995 & -5.19714959166242 & 9.46933648338686 \\
H & 6.36807002544432 & -3.58333314908562 & 8.93394321894350 \\
H & 5.52384407292886 & -6.06117216237887 & 11.12834693757115 \\
H & 4.29290263838955 & -7.33533733302854 & 11.03423008508024 \\
H & 3.86998476547256 & -5.74240203875963 & 11.64449085621212 \\
C & -0.83633309933269 & -5.45537932430110 & 4.46835648648372 \\
C & 1.85978551359097 & -3.83399658458501 & 3.44338557202112 \\
H & 2.87087793707655 & -4.02964387503568 & 3.08529113249729 \\
H & 1.75916346471856 & -2.75566419815619 & 3.59284293100843 \\
H & 1.15550759623938 & -4.13903677132597 & 2.67136843992449 \\
H & -1.01668190724285 & -6.51077650678138 & 4.24578397126605 \\
H & -0.88487821845470 & -4.90490285920138 & 3.53186586598583 \\
H & -1.65308697287446 & -5.10044065293066 & 5.10173822232977 \\
\hline
\end{tabular}




\begin{tabular}{|llll|}
\hline BO & & & \\
BODIPY 43 & & \\
N & 2.76346374557552 & -4.68816663020125 & 5.68240257018154 \\
C & 2.23548764583528 & -5.39146810008084 & 6.68078502345181 \\
N & 4.31306989378232 & -4.91275527513699 & 7.45513383777013 \\
B & 4.28141799065963 & -4.19781469066584 & 6.05425990739799 \\
F & 4.30758091124270 & -2.82867345257400 & 6.13799341269428 \\
F & 5.18730065244028 & -4.69908829083091 & 5.15578263500364 \\
C & 3.14925067073525 & -5.56680934084763 & 7.76524232812222 \\
C & 5.11638997949216 & -5.10557908219530 & 8.54743579488648 \\
C & 4.35304163594178 & -5.89845428080698 & 9.45847286438658 \\
N & 3.10649686134741 & -6.16850008519440 & 8.92880017503860 \\
N & 0.95980347109330 & -5.71802819801890 & 6.42223786080126 \\
C & 0.62606387039417 & -5.17790339768623 & 5.17938032194688 \\
C & 1.78928700657973 & -4.52689580857559 & 4.71490907174800 \\
H & 0.36273308783523 & -6.23401121255652 & 7.04657683709679 \\
C & 6.40806450585900 & -4.67886443270443 & 8.85462960189445 \\
C & 4.90131920718244 & -6.27971159801012 & 10.68607427198594 \\
C & -0.55537106103556 & -5.18027510865100 & 4.45558611635398 \\
C & 1.79472015595234 & -3.87009545100431 & 3.49064226009958 \\
C & 0.61277869882749 & -3.87455438040591 & 2.76544895077918 \\
C & -0.54227944641010 & -4.51194253900618 & 3.23934707693447 \\
H & 2.68513367633921 & -3.37552185381605 & 3.13045424292657 \\
H & 0.57787522682252 & -3.37021616502053 & 1.80992435759681 \\
H & -1.44572040279044 & -5.67200768336376 & 4.82248144842620 \\
H & -1.44424020951008 & -4.48255727892857 & 2.64363168104885 \\
C & 6.92371866245507 & -5.06705883909498 & 10.07733217711140 \\
C & 6.18459254631319 & -5.86124460185568 & 10.97744408391104 \\
H & 6.97739780619124 & -4.07298197861701 & 8.16371295301036 \\
H & 7.92000561587669 & -4.75071314930804 & 10.35634532269582 \\
H & 4.32610852525010 & -6.87986176398208 & 11.37817265395339 \\
H & 6.63641906972210 & -6.14452533085995 & 11.91898016074575 \\
\hline
\end{tabular}




\begin{tabular}{|c|c|c|c|}
\hline 42 & & & \\
\hline $\mathrm{BO}$ & DIPY 44 & & \\
\hline $\mathrm{N}$ & 5.36959450111828 & -4.40839727701271 & 7.39653340716735 \\
\hline $\mathrm{N}$ & 7.29402113343824 & -5.02809643090503 & 8.83106821988144 \\
\hline B & 6.85136886568887 & -4.04836844896787 & 7.70961515669817 \\
\hline $\mathrm{F}$ & 6.94619960009896 & -2.73755680403433 & 8.16720431975246 \\
\hline $\mathrm{F}$ & 7.64619970233250 & -4.22823410085550 & 6.58352826835808 \\
\hline $\mathrm{C}$ & 6.49724350142662 & -5.98697658143549 & 9.44503299799162 \\
\hline $\mathrm{C}$ & 5.16386377373927 & -6.18327949777948 & 9.04424586291090 \\
\hline $\mathrm{C}$ & 4.61751927254109 & -5.40837888603400 & 8.02568988943334 \\
\hline C & 4.59896546133026 & -3.78602894390942 & 6.49530146902663 \\
\hline $\mathrm{C}$ & 3.31915878730444 & -4.37372168657139 & 6.50102064174216 \\
\hline $\mathrm{C}$ & 3.30992248905693 & -5.38650296180598 & 7.44260635749035 \\
\hline $\mathrm{C}$ & 8.50326904015827 & -5.05326099954786 & 9.38570144031275 \\
\hline $\mathrm{C}$ & 8.55012094028915 & -6.03649487580682 & 10.39968226660431 \\
\hline $\mathrm{C}$ & 7.27885797463631 & -6.62236545811620 & 10.43102335141386 \\
\hline $\mathrm{C}$ & 4.34227091771593 & -7.22180632922716 & 9.74767814950753 \\
\hline $\mathrm{C}$ & 5.08415934045050 & -2.66123937649465 & 5.65656418382371 \\
\hline $\mathrm{H}$ & 2.49316329426343 & -4.07257183904899 & 5.87696696811433 \\
\hline $\mathrm{C}$ & 2.13276554197463 & -6.24875986504473 & 7.74837206476177 \\
\hline $\mathrm{H}$ & 6.96826022122664 & -7.41476742471659 & 11.08929798037729 \\
\hline $\mathrm{H}$ & 9.28408540692661 & -4.38417480623599 & 9.06442425733053 \\
\hline $\mathrm{C}$ & 9.68949527249679 & -6.30646537030760 & 11.18497692633658 \\
\hline $\mathrm{H}$ & 5.85025192312374 & -3.01013727747157 & 4.96153169970630 \\
\hline $\mathrm{H}$ & 5.54499273810982 & -1.89096139547167 & 6.27539402980121 \\
\hline $\mathrm{H}$ & 4.26025580005102 & -2.22977051169331 & 5.09076773476361 \\
\hline $\mathrm{H}$ & 1.29144790735947 & -5.96692719099479 & 7.11706615024346 \\
\hline $\mathrm{H}$ & 1.81561395256397 & -6.15101056988109 & 8.78914358892594 \\
\hline $\mathrm{H}$ & 2.34318218178936 & -7.30619442946608 & 7.56990479472003 \\
\hline $\mathrm{H}$ & 4.93812882191937 & -7.77974427921392 & 10.46333440876706 \\
\hline $\mathrm{H}$ & 3.91218855173995 & -7.93000654441270 & 9.04020967449869 \\
\hline $\mathrm{H}$ & 3.51489338085336 & -6.75544580998125 & 10.28515690425181 \\
\hline $\mathrm{C}$ & 10.71152091093317 & -6.45078165092357 & 11.81312617391661 \\
\hline $\mathrm{C}$ & 11.94618982631325 & -6.56732102250860 & 12.50684282413237 \\
\hline $\mathrm{C}$ & 11.98866563791449 & -6.66635035534511 & 13.90462575466789 \\
\hline $\mathrm{C}$ & 13.20629632192649 & -6.73040928654058 & 14.56521735691925 \\
\hline $\mathrm{C}$ & 14.39763359385276 & -6.69998127833012 & 13.84733247255603 \\
\hline $\mathrm{C}$ & 14.36589585833637 & -6.61159696265728 & 12.45921783069009 \\
\hline $\mathrm{C}$ & 13.15353156131480 & -6.54536989718153 & 11.79140379977837 \\
\hline $\mathrm{H}$ & 11.06096097216469 & -6.68279743533628 & 14.46013960549705 \\
\hline $\mathrm{H}$ & 15.28886166217889 & -6.58814082550742 & 11.89449339342991 \\
\hline $\mathrm{H}$ & 13.12277093733252 & -6.46820665321489 & 10.71279058059796 \\
\hline $\mathrm{H}$ & 13.22703053411122 & -6.80175644324631 & 15.64513052110696 \\
\hline $\mathrm{H}$ & 15.34577188789748 & -6.74600221676412 & 14.36677652199412 \\
\hline
\end{tabular}




\begin{tabular}{|c|c|c|c|}
\hline 42 & & & \\
\hline $\mathrm{BO}$ & DIPY 45 & & \\
\hline $\mathrm{N}$ & 5.70039196026296 & -4.41171772328983 & 7.12937611833270 \\
\hline $\mathrm{N}$ & 7.67283514383046 & -4.87220449965552 & 8.56882960320999 \\
\hline B & 7.24703613561183 & -4.20528869329937 & 7.22365972276152 \\
\hline $\mathrm{F}$ & 7.55058788587708 & -2.84684391644370 & 7.24663183966524 \\
\hline $\mathrm{F}$ & 7.86224404140705 & -4.82690125713495 & 6.13956247149379 \\
\hline $\mathrm{C}$ & 6.84941989721877 & -5.63488416463876 & 9.37896509788625 \\
\hline $\mathrm{C}$ & 5.48633829146792 & -5.81328602575745 & 9.09728583835016 \\
\hline $\mathrm{C}$ & 4.91815461118200 & -5.18912196829648 & 7.99082145844211 \\
\hline $\mathrm{C}$ & 4.91768102982295 & -3.92052494039122 & 6.15877788678533 \\
\hline $\mathrm{C}$ & 3.59375413216180 & -4.35272733762408 & 6.37345150436336 \\
\hline $\mathrm{C}$ & 3.57221627597648 & -5.14644325813035 & 7.50640308993641 \\
\hline $\mathrm{C}$ & 8.90559798162520 & -4.83263801042335 & 9.13288332505371 \\
\hline $\mathrm{C}$ & 8.89210859765567 & -5.58573905686702 & 10.33104027437567 \\
\hline $\mathrm{C}$ & 7.61479495763379 & -6.08613985443107 & 10.48109110456240 \\
\hline $\mathrm{C}$ & 4.66037696127652 & -6.66099267706473 & 10.02043898278362 \\
\hline $\mathrm{C}$ & 5.43112471838106 & -3.08211406818135 & 5.04596619525711 \\
\hline $\mathrm{H}$ & 2.74761844655386 & -4.09395809503309 & 5.75726234640845 \\
\hline $\mathrm{C}$ & 2.34743210959595 & -5.79021465451109 & 8.06659290333692 \\
\hline $\mathrm{H}$ & 7.25797969723697 & -6.69524435862338 & 11.29350918111528 \\
\hline C & 9.98719691017338 & -4.13896620343934 & 8.58045026336289 \\
\hline $\mathrm{H}$ & 6.03745535002194 & -2.26201384478752 & 5.42890408882278 \\
\hline $\mathrm{H}$ & 4.60409731929640 & -2.68325584162964 & 4.46130920477775 \\
\hline $\mathrm{H}$ & 6.07545401588898 & -3.67727598291939 & 4.39462143193165 \\
\hline $\mathrm{H}$ & 1.47197556862287 & -5.45878339153864 & 7.51001172708283 \\
\hline $\mathrm{H}$ & 2.19289365009923 & -5.53644933346309 & 9.11689587161182 \\
\hline $\mathrm{H}$ & 2.39075605811430 & -6.88071709723244 & 7.99764167212789 \\
\hline $\mathrm{H}$ & 5.28539212194258 & -7.20593477035639 & 10.72106518779031 \\
\hline $\mathrm{H}$ & 4.06791207372026 & -7.38355463335198 & 9.46245254341686 \\
\hline $\mathrm{H}$ & 3.96711991207328 & -6.04500187903940 & 10.59688242961741 \\
\hline $\mathrm{H}$ & 9.73819004585516 & -5.71874305210427 & 10.98333561901853 \\
\hline C & 10.92935091561756 & -3.54669207168855 & 8.11083663192219 \\
\hline $\mathrm{C}$ & 12.02482903697606 & -2.85450461701464 & 7.54353841541203 \\
\hline $\mathrm{C}$ & 13.33298509916234 & -3.14435794056307 & 7.95852035066087 \\
\hline $\mathrm{C}$ & 14.40901344048991 & -2.48482414882642 & 7.38801443752407 \\
\hline $\mathrm{C}$ & 14.19889988118849 & -1.52146303835115 & 6.40565513093284 \\
\hline $\mathrm{C}$ & 12.90355770836850 & -1.22048868022248 & 5.99535155458768 \\
\hline $\mathrm{C}$ & 11.81968669952103 & -1.87816870700977 & 6.55629751054770 \\
\hline $\mathrm{H}$ & 13.48807595707752 & -3.89428074820250 & 8.72196667906495 \\
\hline $\mathrm{H}$ & 12.73720206231838 & -0.47077012969678 & 5.23286291068444 \\
\hline $\mathrm{H}$ & 10.81016882879315 & -1.65300329909811 & 6.24058760680899 \\
\hline $\mathrm{H}$ & 15.41325980417172 & -2.72408097152732 & 7.71305885605016 \\
\hline $\mathrm{H}$ & 15.04046466572850 & -1.00744505814032 & 5.96101093212315 \\
\hline
\end{tabular}




\begin{tabular}{|c|c|c|c|}
\hline 41 & & & \\
\hline $\mathrm{BO}$ & DIPY 46 & & \\
\hline $\mathrm{N}$ & 6.14880924805480 & -4.56399475627791 & 7.47328640270364 \\
\hline $\mathrm{N}$ & 8.13452984354062 & -4.84054792453342 & 9.00533374273879 \\
\hline B & 7.60651624944588 & -4.14114172279293 & 7.69696199575224 \\
\hline $\mathrm{F}$ & 7.65357745287514 & -2.72944097489238 & 7.86168568844587 \\
\hline $\mathrm{F}$ & 8.41817204278286 & -4.49856825657739 & 6.63166781282000 \\
\hline $\mathrm{C}$ & 7.32171245737830 & -5.67079089151230 & 9.78782967619054 \\
\hline $\mathrm{C}$ & 5.99653536326885 & -5.86989865221319 & 9.49282064773193 \\
\hline $\mathrm{C}$ & 5.39147696009887 & -5.31194579797674 & 8.36729287322012 \\
\hline C & 5.35237670850496 & -4.19076913295136 & 6.44289284429727 \\
\hline $\mathrm{C}$ & 4.06377022697023 & -4.68923840459909 & 6.66925863693586 \\
\hline $\mathrm{C}$ & 4.07217210861337 & -5.39303912827601 & 7.87421438020159 \\
\hline $\mathrm{C}$ & 9.39778837507800 & -4.83916728663493 & 9.48742549951197 \\
\hline $\mathrm{C}$ & 9.44734468436567 & -5.71506393401474 & 10.63319963076935 \\
\hline $\mathrm{C}$ & 8.14057488545935 & -6.22866620751835 & 10.82095498533871 \\
\hline $\mathrm{C}$ & 5.84380734255886 & -3.39813181713219 & 5.28484218989625 \\
\hline $\mathrm{H}$ & 3.21804044938711 & -4.54466534023961 & 6.01582049167165 \\
\hline $\mathrm{C}$ & 2.91845072550123 & -6.09693018934690 & 8.50467573371718 \\
\hline $\mathrm{H}$ & 6.51779820889119 & -3.99527931484041 & 4.66640605820731 \\
\hline $\mathrm{H}$ & 6.40348705529516 & -2.52323681892622 & 5.61641246888082 \\
\hline $\mathrm{H}$ & 5.00483929658402 & -3.07031490559876 & 4.67304490329937 \\
\hline $\mathrm{H}$ & 1.97799874036435 & -5.70360080953963 & 8.11924243187731 \\
\hline $\mathrm{H}$ & 2.91179766588280 & -5.97474894038555 & 9.58959863646140 \\
\hline $\mathrm{H}$ & 2.93745355270812 & -7.16974972861753 & 8.28751089732789 \\
\hline $\mathrm{H}$ & 5.41040800821749 & -6.50192825144291 & 10.14593810484956 \\
\hline $\mathrm{C}$ & 10.47395150468471 & -6.06342854632304 & 11.51875411562552 \\
\hline $\mathrm{C}$ & 10.19563669601709 & -6.94915068548223 & 12.54116695823787 \\
\hline $\mathrm{C}$ & 8.90244463283259 & -7.47307686619195 & 12.71247829010959 \\
\hline $\mathrm{C}$ & 7.86942683160223 & -7.11286642597909 & 11.86925706351080 \\
\hline $\mathrm{H}$ & 11.46245964204708 & -5.64227112733298 & 11.41854000547940 \\
\hline $\mathrm{H}$ & 10.98036407082308 & -7.23743195155269 & 13.22775336133767 \\
\hline $\mathrm{H}$ & 8.71377511804003 & -8.16266105994917 & 13.52482496873483 \\
\hline $\mathrm{H}$ & 6.87483695443713 & -7.51129121750537 & 12.02172364198636 \\
\hline $\mathrm{C}$ & 10.48931926890237 & -4.07102536617927 & 8.97360959061370 \\
\hline $\mathrm{N}$ & 10.34085785974050 & -2.91193471596906 & 8.24442772077150 \\
\hline $\mathrm{C}$ & 11.86557991319305 & -4.29186190306761 & 9.09123367399006 \\
\hline $\mathrm{C}$ & 12.52723621396551 & -3.25325771599494 & 8.41453533982182 \\
\hline $\mathrm{C}$ & 11.55072980771136 & -2.41259330790181 & 7.90799536223209 \\
\hline $\mathrm{H}$ & 12.32588330852292 & -5.14012746738365 & 9.56399761069345 \\
\hline $\mathrm{H}$ & 9.44325369660087 & -2.51428769855738 & 7.99976580090167 \\
\hline $\mathrm{H}$ & 13.58974590453643 & -3.12289965147561 & 8.29789719698177 \\
\hline $\mathrm{H}$ & 11.63850092451578 & -1.49800510631371 & 7.34763256612524 \\
\hline
\end{tabular}




\begin{tabular}{|c|c|c|c|}
\hline 71 & & & \\
\hline & DIPY 47 & & \\
\hline $\mathrm{N}$ & 5.96182683479308 & -3.74244562086110 & 7.01573359500377 \\
\hline $\mathrm{N}$ & 8.09894960837740 & -4.20378743354669 & 8.25136479389091 \\
\hline B & 7.43423431243276 & -3.33001050322928 & 7.14029868357997 \\
\hline $\mathrm{F}$ & 7.46630450196689 & -1.96270136587295 & 7.54029343384928 \\
\hline $\mathrm{F}$ & 8.12826557012169 & -3.48573874444041 & 5.94380162911941 \\
\hline $\mathrm{C}$ & 7.36795797871463 & -5.09342074217936 & 9.04472497924067 \\
\hline $\mathrm{C}$ & 6.00664045095011 & -5.23687471795852 & 8.89688338742872 \\
\hline $\mathrm{C}$ & 5.29337963379148 & -4.55855817501861 & 7.90662155853179 \\
\hline C & 5.07351272286140 & -3.26420086164164 & 6.09863772245286 \\
\hline $\mathrm{C}$ & 3.79879339632467 & -3.77717649592132 & 6.41355641113877 \\
\hline $\mathrm{C}$ & 3.92045192925867 & -4.57473326867260 & 7.54352557043650 \\
\hline $\mathrm{C}$ & 9.41783670762082 & -4.26626030951635 & 8.55829260965705 \\
\hline $\mathrm{C}$ & 9.58657563575936 & -5.24886941942082 & 9.59470812801616 \\
\hline $\mathrm{C}$ & 8.29987806343382 & -5.76279683069685 & 9.90351315429615 \\
\hline $\mathrm{C}$ & 5.53605620867508 & -2.39443729249007 & 5.06265588857665 \\
\hline $\mathrm{H}$ & 2.88835822684224 & -3.55649197855437 & 5.88107660561629 \\
\hline $\mathrm{C}$ & 2.82387321944356 & -5.30326797442028 & 8.24258757167040 \\
\hline $\mathrm{H}$ & 1.85341152375152 & -4.95317441671175 & 7.89219706032824 \\
\hline $\mathrm{H}$ & 2.86363025717804 & -5.14907620604829 & 9.32341220982856 \\
\hline $\mathrm{H}$ & 2.87482047394464 & -6.38012535353168 & 8.05460403978705 \\
\hline $\mathrm{H}$ & 5.48294630507586 & -5.92183403608350 & 9.54947507212504 \\
\hline $\mathrm{C}$ & 10.70770620577587 & -5.65733813617742 & 10.32539775251012 \\
\hline $\mathrm{C}$ & 10.54529857170571 & -6.61137630853385 & 11.30983805321062 \\
\hline $\mathrm{C}$ & 9.27505473551946 & -7.14520185114009 & 11.59288547266715 \\
\hline $\mathrm{C}$ & 8.15001471972078 & -6.72220193061741 & 10.91014655726144 \\
\hline $\mathrm{H}$ & 11.67734797673321 & -5.22578939122786 & 10.13146678125117 \\
\hline $\mathrm{H}$ & 11.40142745037535 & -6.94896673584205 & 11.87846122090639 \\
\hline $\mathrm{H}$ & 9.17790947960485 & -7.89091732273074 & 12.37131375476137 \\
\hline $\mathrm{H}$ & 7.17689858673274 & -7.12473027497818 & 11.15985566026773 \\
\hline $\mathrm{C}$ & 10.41791365501696 & -3.41761000743750 & 7.98048429783231 \\
\hline $\mathrm{N}$ & 10.13612933884275 & -2.10958442825817 & 7.63305666669400 \\
\hline C & 11.74372970941109 & -3.66092963809599 & 7.59768200528218 \\
\hline $\mathrm{C}$ & 12.22324213367868 & -2.46396761319219 & 7.01886438961351 \\
\hline $\mathrm{C}$ & 11.20432934453645 & -1.52470161925412 & 7.04690710536596 \\
\hline $\mathrm{C}$ & 12.48956998588591 & -4.95664072360260 & 7.62994631264729 \\
\hline $\mathrm{H}$ & 9.21922478755643 & -1.69426188529797 & 7.72619480504183 \\
\hline $\mathrm{H}$ & 13.20266566504570 & -2.31008757515066 & 6.59558490797297 \\
\hline C & 11.13491519454191 & -0.12549559831387 & 6.54276850338014 \\
\hline $\mathrm{H}$ & 12.09839862995572 & 0.18415725923332 & 6.14077625444323 \\
\hline $\mathrm{H}$ & 10.85293916845049 & 0.57362386113730 & 7.33442464295974 \\
\hline $\mathrm{H}$ & 10.38906453410881 & -0.02987392432964 & 5.74866603288132 \\
\hline $\mathrm{H}$ & 11.81647177157257 & -5.80652698811394 & 7.72697693983449 \\
\hline $\mathrm{H}$ & 13.20875706381909 & -5.00411533892009 & 8.45249643388643 \\
\hline $\mathrm{H}$ & 13.05757124529412 & -5.08362605785600 & 6.70686639014508 \\
\hline C & 4.93436253378605 & -2.03582897483175 & 3.91422311078705 \\
\hline $\mathrm{C}$ & 5.62140657609479 & -1.23879535878318 & 2.90591935813856 \\
\hline $\mathrm{C}$ & 7.01435005083304 & -1.09463528595379 & 2.92620257965845 \\
\hline $\mathrm{C}$ & 7.68101724436004 & -0.30865119632876 & 2.00498125681639 \\
\hline C & 6.96252518904277 & 0.35628750729900 & 1.00962786076682 \\
\hline $\mathrm{C}$ & 5.57782517562421 & 0.19600576649943 & 0.94189101908364 \\
\hline C & 4.92524378103287 & -0.59593189009265 & 1.87041729116444 \\
\hline
\end{tabular}




\begin{tabular}{|llll|}
\hline H & 6.53381668166619 & -2.02306327775484 & 5.22932499469857 \\
H & 3.92092475284016 & -2.35416992765883 & 3.69331212338728 \\
H & 7.60257375327103 & -1.62853554083575 & 3.65886266100725 \\
H & 5.03466929281238 & 0.70140253705784 & 0.15510831115383 \\
H & 3.85022388610777 & -0.71499506670833 & 1.80187750644654 \\
H & 8.75594097109482 & -0.23911505854747 & 2.07191733452634 \\
O & 7.53235854809004 & 1.15787437571770 & 0.06261257830008 \\
C & 8.92081848396265 & 1.33561707572852 & 0.14149552953288 \\
H & 9.20039232260490 & 1.80832441661149 & 1.09050324510536 \\
C & 9.40327837025621 & 2.19343853174531 & -1.01424937290995 \\
H & 9.44044625296499 & 0.37123010101065 & 0.08989154990901 \\
O & 8.71438556473978 & 2.58058760864962 & -1.90887168860568 \\
O & 10.72553315150903 & 2.48960195193523 & -1.00145674069916 \\
C & 11.59854698916229 & 2.13093899580934 & 0.08693164970512 \\
C & 13.02126890366283 & 2.25462163345157 & -0.40950744673590 \\
H & 11.40308665974819 & 1.10986172681095 & 0.41942153206009 \\
H & 11.41341471715060 & 2.80965187932813 & 0.92368255210256 \\
H & 13.19697185051517 & 1.57080681152010 & -1.23925138305500 \\
H & 13.71622231765203 & 2.00699038922690 & 0.39493097574351 \\
H & 13.22555246421279 & 3.26934424460961 & -0.75125343347879 \\
\hline
\end{tabular}




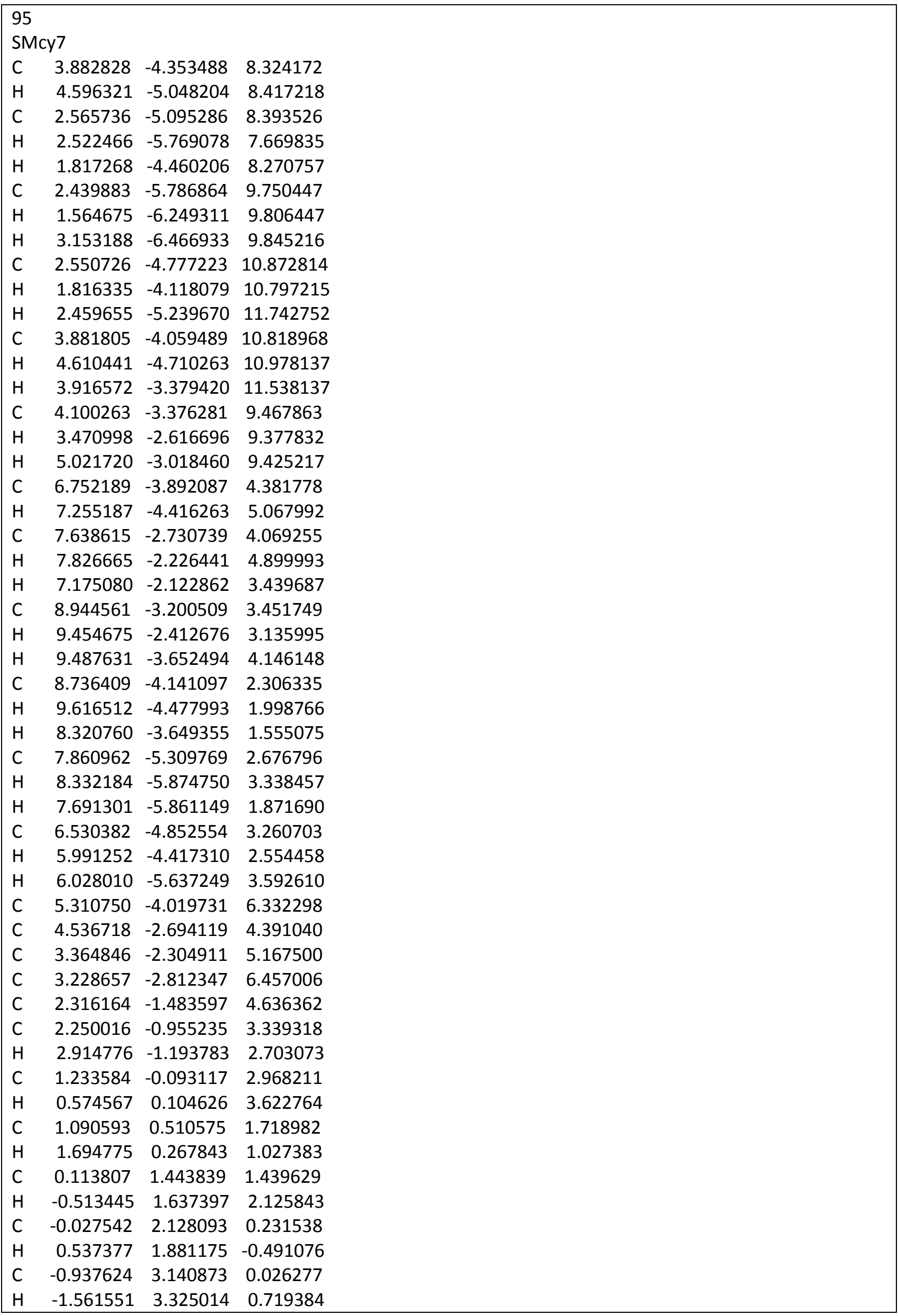




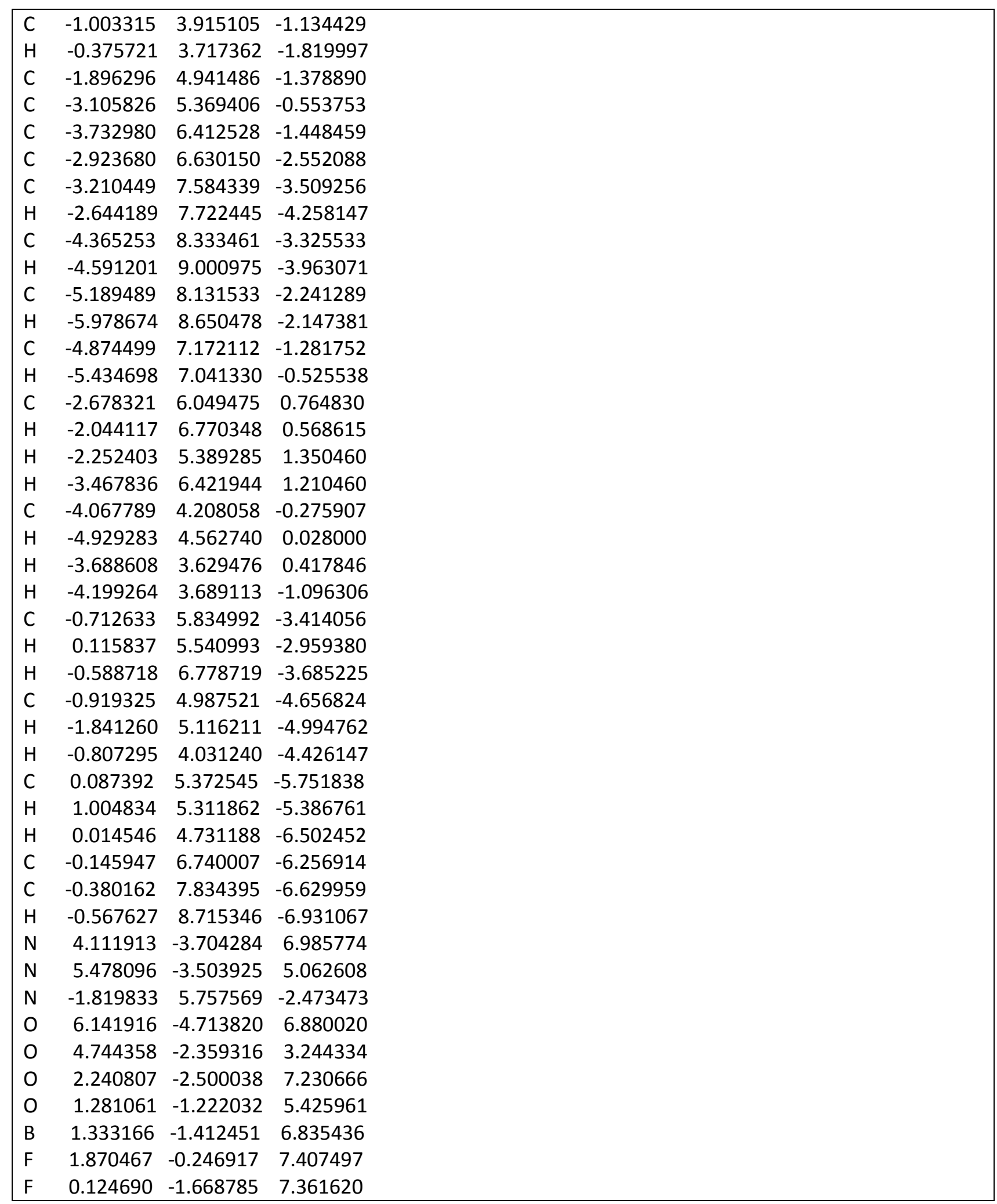




\begin{tabular}{|c|c|c|c|}
\hline \\
\hline \\
\hline & \multicolumn{3}{|c|}{ DT-1 } \\
\hline \multicolumn{4}{|c|}{ Br 19.33666446744866} \\
\hline \multicolumn{2}{|r|}{ Br 22.34624194105621 } & 8.97232565344089 & 9.14607 \\
\hline \multicolumn{2}{|c|}{ H 20.23993897213305} & 10.13175586671681 & 14.72950949652855 \\
\hline \multicolumn{2}{|r|}{$\mathrm{Br} 18.13633702729357$} & 19.75339119250620 & 17.76900918227074 \\
\hline \multicolumn{2}{|r|}{ C 20.19321196345757} & 15.68583885321558 & 3.78200172594863 \\
\hline \multirow{2}{*}{\multicolumn{2}{|c|}{$\begin{array}{ll}\text { C } & 21.37094679123954 \\
\text { H } & 21.95981460771049\end{array}$}} & 15.03890133526739 & 3.52997806480244 \\
\hline & & 15.19777016649800 & 2.63986352449565 \\
\hline \multicolumn{2}{|r|}{ C 21.71917907368370} & 14.15595756575061 & 4.57943951407152 \\
\hline \multicolumn{2}{|r|}{ H 22.62079407099463} & 13.56037796525561 & 4.58605806125502 \\
\hline & 14.15070841271328 & 5.61931855323792 \\
\hline \multirow{2}{*}{\multicolumn{2}{|c|}{$\begin{array}{ll}\text { C } & 20.84843326907895 \\
\text { C } & 20.27316570181169\end{array}$}} & 13.36442296881723 & 6.82314639992201 \\
\hline & & 13.64135684609500 & 8.04144163467736 \\
\hline \multicolumn{2}{|r|}{ H 19.75508022880735} & 14.560366644 & 16692869 \\
\hline & 20.48153426783754 & 12.585612061 & 793724 \\
\hline & 21.21937924548712 & 11.50574563444358 & 8.40484254739455 \\
\hline$c$ & 21.32935096759587 & 10.53475203845951 & 9.37646661685112 \\
\hline & 20.65208770309553 & 11.03785908893880 & 10.53880163866382 \\
\hline & 20.526265 & 10.5204273 & 198 \\
\hline & 20.0882906 & 11.3 & 12. \\
\hline & 20.02798377739289 & 11.0808114 & 2314955 \\
\hline$c$ & 19.701172 & 489977616 & 19725798 \\
\hline$c$ & 19.52550215518049 & 13.25213011627721 & 13.83655085986279 \\
\hline C & 19.20171393780602 & 14.53690287557875 & 14.31 \\
\hline $\mathrm{H}$ & 19.038506 & 15.3974394 & 382 \\
\hline C & 19.136050 & 14.5 & 15 \\
\hline & 18.86992195553857 & 15.67254893 & 5222850896381 \\
\hline & 18.7578012 & 15.67860088 & 17.927 \\
\hline C & 18.44083360289049 & 17.92011358702987 & 5577022 \\
\hline & 20.9124516 & 9.116901574 & 487 \\
\hline & 22.0 & 8.823 & $12 . \varepsilon$ \\
\hline & 22.607146 & 9.62 & 509 \\
\hline & 22.48392962904265 & 7.51494856408921 & 4574410393 \\
\hline & 23.38836588656978 & 7.28053851052036 & 13.52758924434600 \\
\hline & 21.78530435 & 6.479262045 & 477755180 \\
\hline & 20.6 & 6.7627 & 492 \\
\hline & 20.062754 & 5.96 & 3324 \\
\hline & 20.18730848525654 & 8.073448002 & 11.52 \\
\hline & 19.30496551929654 & 8.29857813225184 & 10.94531910488829 \\
\hline & 22.36681395186917 & 5.11057166901068 & 12.42294869868646 \\
\hline & 22.093057 & 2.85 & 7818 \\
\hline & 22.958990 & 2.83 & 11.1 \\
\hline & 21.29220686478456 & 2.24805094818378 & 11.37397791274080 \\
\hline & 22.39233290074079 & 2.49726987364611 & 12.76732477648552 \\
\hline & 18.04624228292252 & 13.22583898692647 & 11.01448336512184 \\
\hline & 19.901943 & 14.552 & 11. \\
\hline & 20.16225595642104 & 12.31099997223232 & 10.23419924319214 \\
\hline & 19.74719993788360 & 12.67810139092560 & 12.63694448824931 \\
\hline & 23.45032013036510 & 4.85079114427580 & 12.88808630010666 \\
\hline & 21.56020031991942 & 4.18547375838355 & 11.86955862144602 \\
\hline & 19.49505570275357 & 15.23472959000660 & 5.29039673418121 \\
\hline
\end{tabular}




\begin{tabular}{|llll|}
\hline S & 21.68429139222183 & 11.80862132028069 & 6.77242897388460 \\
S & 19.46672462223757 & 12.98697671324596 & 16.42805339947753 \\
S & 18.66490016224893 & 17.28297918804017 & 15.92140634401973 \\
C & 18.51143576303929 & 16.95753944009808 & 18.47483750982829 \\
H & 18.85145658584990 & 14.78495807070792 & 18.52750015195420 \\
H & 18.39077879024845 & 17.16416574978817 & 19.52721445208102 \\
\hline
\end{tabular}




\begin{tabular}{|c|c|c|c|}
\hline 94 & & & \\
\hline BD- & & & \\
\hline $\mathrm{C}$ & -4.26265108889794 & 5.03328676439349 & -3.28911415542909 \\
\hline $\mathrm{N}$ & -5.06075189197238 & 4.30420789389914 & -2.47117587899638 \\
\hline C & -2.92642994594015 & 4.74285228861730 & -3.60102907839339 \\
\hline $\mathrm{C}$ & -2.38024081418005 & 3.53648988629605 & -3.10704653068440 \\
\hline $\mathrm{N}$ & -3.11352854406203 & 2.76488664074962 & -2.19833308164667 \\
\hline B & -4.52166069346738 & 3.12040741494178 & -1.62142078935097 \\
\hline $\mathrm{F}$ & -4.42953438834268 & 3.54189711375522 & -0.29321061616546 \\
\hline $\mathrm{F}$ & -5.36449401190323 & 2.01710420732041 & -1.72668191186836 \\
\hline C & -4.98719175647327 & 6.15522571480914 & -3.77118529479498 \\
\hline $\mathrm{C}$ & -6.27118132083091 & 6.07290637142269 & -3.16683011255671 \\
\hline $\mathrm{C}$ & -6.28384498323768 & 4.93248842835182 & -2.38016879096375 \\
\hline $\mathrm{C}$ & -1.19523562920987 & 2.82634823762594 & -3.40306635760309 \\
\hline $\mathrm{C}$ & -1.21509811426638 & 1.65955603952749 & -2.65731318156333 \\
\hline $\mathrm{C}$ & -2.41116237795114 & 1.64128435845305 & -1.91841085813233 \\
\hline $\mathrm{C}$ & -7.40393196822392 & 4.36326250225610 & -1.57722428415305 \\
\hline $\mathrm{C}$ & -8.26332347958443 & 3.38657092855935 & -2.39458002448978 \\
\hline $\mathrm{H}$ & -8.02249528053267 & 5.18530847975677 & -1.21248226893462 \\
\hline $\mathrm{H}$ & -6.99720976621362 & 3.85010910023691 & -0.70647751471986 \\
\hline $\mathrm{H}$ & -8.74663704976372 & 3.90006060576607 & -3.22764221294388 \\
\hline $\mathrm{H}$ & -9.03907390103877 & 2.94601808712436 & -1.76600007846811 \\
\hline $\mathrm{H}$ & -7.64755145694639 & 2.58078173997825 & -2.79173938967039 \\
\hline $\mathrm{C}$ & -2.85947194092843 & 0.56704710049043 & -0.98494487915017 \\
\hline $\mathrm{C}$ & -2.83774027949531 & 0.96904780671324 & 0.49872434855524 \\
\hline $\mathrm{H}$ & -2.19697144990621 & -0.28623631569179 & -1.14099247545241 \\
\hline $\mathrm{H}$ & -3.86722767519480 & 0.25122542930690 & -1.25674096180510 \\
\hline $\mathrm{H}$ & -1.84418170842491 & 1.31067730012943 & 0.79608911698752 \\
\hline $\mathrm{H}$ & -3.11001893267224 & 0.11189858167851 & 1.11678738805700 \\
\hline $\mathrm{H}$ & -3.54267534753279 & 1.77122202792816 & 0.69765581622094 \\
\hline $\mathrm{C}$ & -4.37468665905577 & 7.05774271298277 & -4.65538007792744 \\
\hline $\mathrm{C}$ & -3.02612112422138 & 6.82567677396388 & -5.00624793418441 \\
\hline $\mathrm{C}$ & -2.27111533871916 & 5.72814857860702 & -4.42938472547831 \\
\hline $\mathrm{C}$ & -2.37674353990878 & 7.64242658688890 & -5.95389401691286 \\
\hline $\mathrm{C}$ & -1.04672991531368 & 7.51487135767374 & -6.30930847301053 \\
\hline $\mathrm{C}$ & -0.23886562142100 & 6.60157074890729 & -5.51755977954149 \\
\hline $\mathrm{C}$ & -0.90705453123369 & 5.72176975143052 & -4.64598959223816 \\
\hline $\mathrm{C}$ & -0.44247444299962 & 8.29638958581514 & -7.36782256520488 \\
\hline $\mathrm{C}$ & 1.16823357361571 & 6.55742901914257 & -5.63771779167012 \\
\hline $\mathrm{C}$ & 1.76288274625883 & 7.37618877310845 & -6.61012210446279 \\
\hline $\mathrm{C}$ & 0.95837292549612 & 8.20437406622129 & -7.43719875853462 \\
\hline $\mathrm{H}$ & -7.09143612573578 & 6.76047772699687 & -3.28504742516248 \\
\hline $\mathrm{H}$ & -0.44709476218808 & 3.09831228329227 & -4.12529345477087 \\
\hline $\mathrm{H}$ & -0.47501820616235 & 0.87616276976485 & -2.65419557107524 \\
\hline $\mathrm{C}$ & -5.13486058953216 & 8.21164433291433 & -5.17398932395215 \\
\hline $\mathrm{H}$ & -0.30659466686264 & 5.01260477667528 & -4.10621486857889 \\
\hline $\mathrm{H}$ & -2.95981689716808 & 8.42046078498146 & -6.41184820453417 \\
\hline $\mathrm{C}$ & 2.01050924839686 & 5.71202660781145 & -4.76920144578568 \\
\hline $\mathrm{C}$ & -1.09338588354903 & 9.11610402230115 & -8.31767210193396 \\
\hline $\mathrm{N}$ & -0.33041470164700 & 9.91605219792539 & -9.17729278839908 \\
\hline B & 1.22745524215816 & 9.93814242301146 & -9.30688956105026 \\
\hline $\mathrm{N}$ & 1.76098167005302 & 8.89809558629290 & -8.28351442335893 \\
\hline $\mathrm{C}$ & 3.07165978439905 & 8.55202451974787 & -8.02514587604723 \\
\hline
\end{tabular}




\begin{tabular}{|c|c|c|c|}
\hline $\mathrm{C}$ & 3.10669191300882 & 7.62154624152704 & -7.00211657278289 \\
\hline C & 4.19139969957138 & 9.16823786558268 & -8.78995432875366 \\
\hline C & -2.45213251279958 & 9.32984593860950 & -8.64762362541691 \\
\hline C & -2.49221602297638 & 10.27008694908679 & -9.66260367954262 \\
\hline C & -1.16567790331965 & 10.62231281253496 & -9.97184346430659 \\
\hline C & -0.70932899065873 & 11.63145406094174 & -10.97054480883470 \\
\hline $\mathrm{H}$ & -1.46822350523371 & 11.69552928735133 & -11.75358545013701 \\
\hline $\mathrm{H}$ & 0.21332185227373 & 11.29297597675349 & -11.43950538878718 \\
\hline C & -0.49086254787302 & 13.02409116530324 & -10.35191386852991 \\
\hline $\mathrm{H}$ & -1.41069921552352 & 13.39997138958286 & -9.89974637336893 \\
\hline $\mathrm{H}$ & -0.17158474688299 & 13.72741532995221 & -11.12262065167123 \\
\hline $\mathrm{H}$ & 0.27918372285170 & 12.98255588423310 & -9.58433830390000 \\
\hline $\mathrm{H}$ & 4.12040611136788 & 10.25388976721441 & -8.67173014851882 \\
\hline $\mathrm{H}$ & 4.02035396262761 & 8.97758647556300 & -9.85358734263393 \\
\hline C & 5.57827320632075 & 8.68234584584546 & -8.38450257316954 \\
\hline $\mathrm{H}$ & 5.78016303227689 & 8.88650843998106 & -7.33115488396041 \\
\hline $\mathrm{H}$ & 6.33910773919953 & 9.19393764197810 & -8.97283237137992 \\
\hline $\mathrm{H}$ & 5.69193329424788 & 7.60979924820699 & -8.55618993482451 \\
\hline $\mathrm{F}$ & 1.60656474145683 & 9.57189279442509 & -10.60095554805419 \\
\hline $\mathrm{F}$ & 1.71620188280602 & 11.20842410177166 & -9.00380094871568 \\
\hline $\mathrm{H}$ & -3.30175358500953 & 8.82104127092838 & -8.23050949928388 \\
\hline $\mathrm{H}$ & -3.36748523944424 & 10.65770086739721 & -10.15733462072476 \\
\hline $\mathrm{H}$ & 3.99118989273514 & 7.17994817310033 & -6.58006767753930 \\
\hline C & -6.32354840138921 & 8.01142753409703 & -5.88291714765412 \\
\hline C & -7.03894947688057 & 9.09411832388493 & -6.37508767524802 \\
\hline C & -6.59142211272123 & 10.39187387921706 & -6.15107199280920 \\
\hline C & -5.41864460690902 & 10.60226412004677 & -5.43554270354082 \\
\hline C & -4.69394611127762 & 9.52023152383787 & -4.95581047204981 \\
\hline C & 2.93729880552861 & 4.82080902298878 & -5.32208357617248 \\
\hline C & 3.73384314615638 & 4.03254130223298 & -4.50343025062778 \\
\hline C & 3.63451990900157 & 4.13255202969901 & -3.11946245062398 \\
\hline C & 2.72842636151270 & 5.02437757935176 & -2.55827736789512 \\
\hline C & 1.92120369239907 & 5.80345371112256 & -3.37503059757647 \\
\hline $\mathrm{H}$ & 3.01781532281694 & 4.73383438993627 & -6.39770480748254 \\
\hline $\mathrm{H}$ & 4.43396918242776 & 3.33755281902772 & -4.94835460161126 \\
\hline $\mathrm{H}$ & 4.26105155797850 & 3.52110383993953 & -2.48386695411945 \\
\hline $\mathrm{H}$ & 2.64904264152796 & 5.11907875608952 & -1.48297199765490 \\
\hline $\mathrm{H}$ & 1.22805737407634 & 6.50603855761901 & -2.93287150376035 \\
\hline $\mathrm{H}$ & -6.67101212641783 & 7.00228969758203 & -6.06227700126110 \\
\hline $\mathrm{H}$ & -7.94852708524998 & 8.92434770880183 & -6.93600116012704 \\
\hline $\mathrm{H}$ & -7.15363953103823 & 11.23312558346119 & -6.53354400354905 \\
\hline $\mathrm{H}$ & -5.06386525483422 & 11.60722323748010 & -5.24858070811667 \\
\hline $\mathrm{H}$ & -3.78461645519694 & 9.68494813486080 & -4.39430604135302 \\
\hline
\end{tabular}




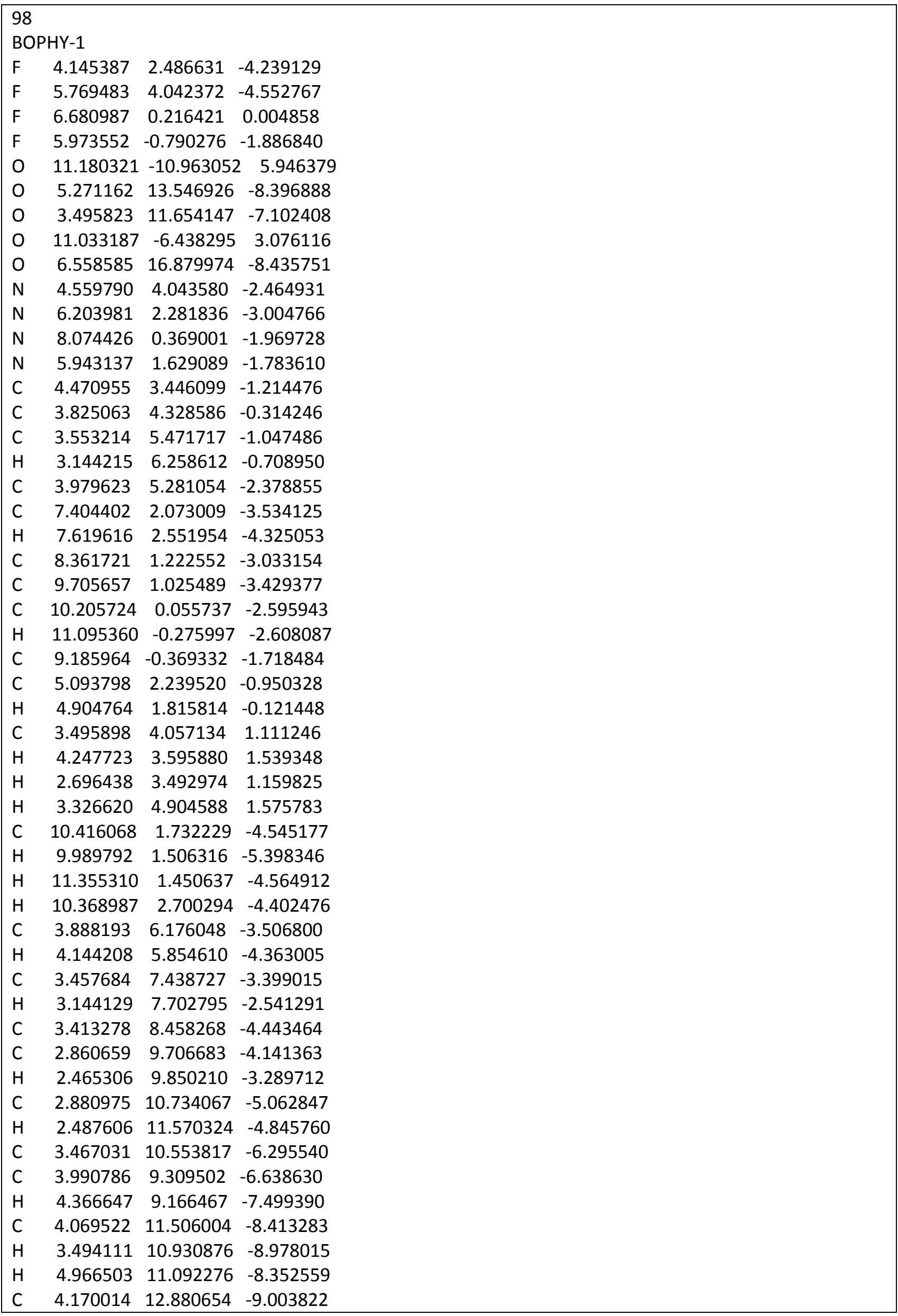




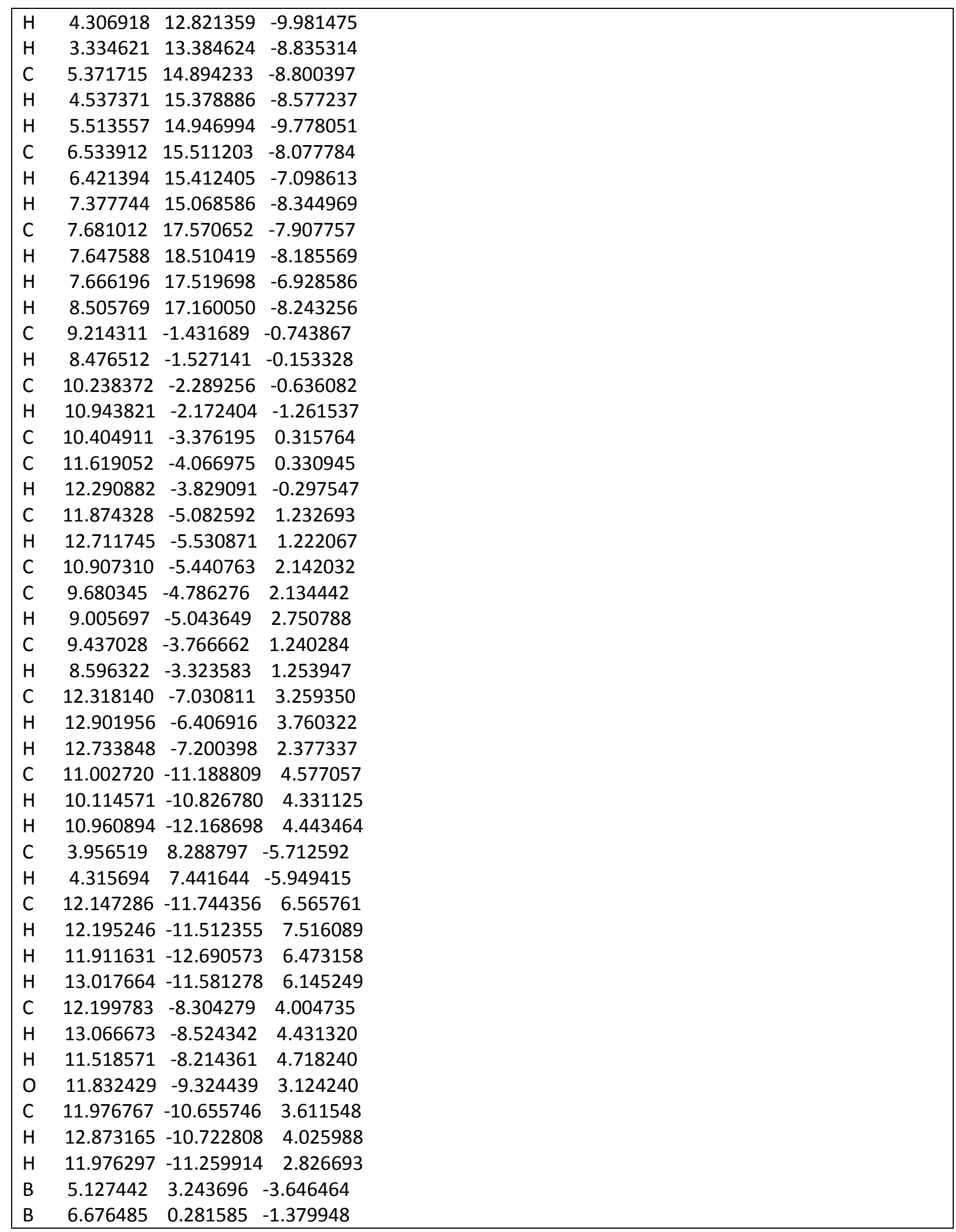


1. Graser, M.; Kopacka, H.; Wurst, K.; Ruetz, M.; Kreutz, C. R.; Müller, T.; Hirtenlehner, C.; Monkowius, U.; Knör, G.; Bildstein, B., Efficient fluorophores based on pyridyl-enolato and enamido difluoroboron complexes: Simple alternatives to boron-dipyrromethene (bodipy) dyes. Inorganica Chimica Acta 2013, 405, 116-120.

2. Tanaka, K.; Yamane, H.; Yoshii, R.; Chujo, Y., Efficient light absorbers based on thiophenefused boron dipyrromethene (BODIPY) dyes. Bioorg Med Chem 2013, 21 (10), 2715-9.

3. Jiang, X.-D.; Zhang, H.; Zhang, Y.; Zhao, W., Development of non-symmetric thiophene-fused BODIPYs. Tetrahedron 2012, 68 (47), 9795-9801.

4. Arroyo, I. J.; Hu, R.; Merino, G.; Tang, B. Z.; Pena-Cabrera, E., The smallest and one of the brightest. Efficient preparation and optical description of the parent borondipyrromethene system. $J$ Org Chem 2009, 74 (15), 5719-22.

5. Banuelos, J.; Martin, V.; Gomez-Duran, C. F.; Arroyo Cordoba, I. J.; Pena-Cabrera, E.; GarciaMoreno, I.; Costela, A.; Perez-Ojeda, M. E.; Arbeloa, T.; Lopez Arbeloa, I., New 8-amino-BODIPY derivatives: surpassing laser dyes at blue-edge wavelengths. Chemistry 2011, 17 (26), 7261-70. 6. Liras, M.; Prieto, J. B.; Pintado-Sierra, M.; Arbeloa, F. L.; Garcia-Moreno, I.; Costela, A.; Infantes, L.; Sastre, R.; Amat-Guerri, F., Synthesis, photophysical properties, and laser behavior of 3amino and 3-acetamido BODIPY dyes. Org Lett 2007, 9 (21), 4183-6.

7. Loudet, A.; Burgess, K., BODIPY dyes and their derivatives: syntheses and spectroscopic properties. Chem Rev 2007, 107 (11), 4891-932.

8. Pavlopoulos, T. G.; Boyer, J. H.; Shah, M.; Thangaraj, K.; Soong, M. L., Laser action from 2,6,8position trisubstituted 1,3,5,7-tetramethylpyrromethene-BF(2) complexes: part 1. Appl Opt 1990, 29 (27), 3885-6.

9. $\quad$ Rihn, S.; Retailleau, P.; Bugsaliewicz, N.; Nicola, A. D.; Ziessel, R., Versatile synthetic methods for the engineering of thiophene-substituted Bodipy dyes. Tetrahedron Lett 2009, 50 (50), 70087013.

10. Yu, C.; Jiao, L.; Yin, H.; Zhou, J.; Pang, W.; Wu, Y.; Wang, Z.; Yang, G.; Hao, E., $\alpha$-/ $\beta$-Formylated Boron-Dipyrrin (BODIPY) Dyes: Regioselective Syntheses and Photophysical Properties. Eur J Org Chem 2011, 2011 (28), 5460-5468.

11. Leen, V.; Miscoria, D.; Yin, S.; Filarowski, A.; Ngongo, J. M.; Van der Auweraer, M.; Boens, N.; Dehaen, W., 1,7-Disubstituted boron dipyrromethene (BODIPY) dyes: synthesis and spectroscopic properties. J Org Chem 2011, 76 (20), 8168-76.

12. Wittmershaus, B. P.; Skibicki, J. J.; McLafferty, J. B.; Zhang, Y. Z.; Swan, S., Spectral properties of single BODIPY dyes in polystyrene microspheres and in solutions. Journal of Fluorescence 2001,11 (2), 119-128.

13. Ulrich, G.; Goeb, S.; De Nicola, A.; Retailleau, P.; Ziessel, R., Chemistry at boron: synthesis and properties of red to near-IR fluorescent dyes based on boron-substituted diisoindolomethene frameworks. J Org Chem 2011, 76 (11), 4489-505.

14. Jiao, L.; Yu, C.; Liu, M.; Wu, Y.; Cong, K.; Meng, T.; Wang, Y.; Hao, E., Synthesis and functionalization of asymmetrical benzo-fused BODIPY dyes. J Org Chem 2010, 75 (17), 6035-8.

15. Umezawa, K.; Matsui, A.; Nakamura, Y.; Citterio, D.; Suzuki, K., Bright, color-tunable fluorescent dyes in the Vis/NIR region: establishment of new "tailor-made" multicolor fluorophores based on borondipyrromethene. Chemistry 2009, 15 (5), 1096-106.

16. Zhou, Y.; Xiao, Y.; Li, D.; Fu, M.; Qian, X., Novel fluorescent fluorine-boron complexes: synthesis, crystal structure, photoluminescence, and electrochemistry properties. J Org Chem 2008, 73 (4), 1571-4.

17. Kubota, Y.; Tsuzuki, T.; Funabiki, K.; Ebihara, M.; Matsui, M., Synthesis and fluorescence properties of a pyridomethene-BF2 complex. Org Lett 2010, 12 (18), 4010-3.

18. Tomimori, Y.; Okujima, T.; Yano, T.; Mori, S.; Ono, N.; Yamada, H.; Uno, H., Synthesis of $\pi-$ expanded O-chelated boron-dipyrromethene as an NIR dye. Tetrahedron 2011, 67 (18), 3187-3193.

19. Kubota, Y.; Hara, H.; Tanaka, S.; Funabiki, K.; Matsui, M., Synthesis and fluorescence properties of novel pyrazine-boron complexes bearing a beta-iminoketone ligand. Org Lett 2011, 13 (24), 6544-7. 
20. Nakamura, M.; Tahara, H.; Takahashi, K.; Nagata, T.; Uoyama, H.; Kuzuhara, D.; Mori, S.; Okujima, T.; Yamada, H.; Uno, H., pi-Fused bis-BODIPY as a candidate for NIR dyes. Org Biomol Chem 2012, 10 (34), 6840-9.

21. Khan, T. K.; Ravikanth, M., 3-(Pyridine-4-thione)BODIPY as a chemodosimeter for detection of $\mathrm{Hg}(\mathrm{II})$ ions. Dyes and Pigments 2012, 95 (1), 89-95.

22. Rohand, T.; Baruah, M.; Qin, W.; Boens, N.; Dehaen, W., Functionalisation of fluorescent BODIPY dyes by nucleophilic substitution. Chem Commun (Camb) 2006, (3), 266-8.

23. Uppal, T.; Hu, X.; Fronczek, F. R.; Maschek, S.; Bobadova-Parvanova, P.; Vicente, M. G., Synthesis, computational modeling, and properties of benzo-appended BODIPYs. Chemistry 2012, 18 (13), 3893-905.

24. Leen, V.; Leemans, T.; Boens, N.; Dehaen, W., 2-and 3-Monohalogenated BODIPY Dyes and Their Functionalized Analogues: Synthesis and Spectroscopy. Eur J Org Chem 2011, (23), 4386-4396. 25. Yu, C.; Xu, Y.; Jiao, L.; Zhou, J.; Wang, Z.; Hao, E., Isoindole-BODIPY dyes as red to nearinfrared fluorophores. Chemistry 2012, 18 (21), 6437-42. 